University of New Orleans

ScholarWorks@UNO

$5-8-2004$

\title{
A Numerical Method for Computing Radially Symmetric Solutions of a Dissipative Nonlinear Modified Klein-Gordon Equation
}

Jorge Macias Diaz

University of New Orleans

Follow this and additional works at: https://scholarworks.uno.edu/td

\section{Recommended Citation}

Macias Diaz, Jorge, "A Numerical Method for Computing Radially Symmetric Solutions of a Dissipative Nonlinear Modified Klein-Gordon Equation" (2004). University of New Orleans Theses and Dissertations. 167.

https://scholarworks.uno.edu/td/167

This Thesis is protected by copyright and/or related rights. It has been brought to you by ScholarWorks@UNO with permission from the rights-holder(s). You are free to use this Thesis in any way that is permitted by the copyright and related rights legislation that applies to your use. For other uses you need to obtain permission from the rightsholder(s) directly, unless additional rights are indicated by a Creative Commons license in the record and/or on the work itself.

This Thesis has been accepted for inclusion in University of New Orleans Theses and Dissertations by an authorized administrator of ScholarWorks@UNO. For more information, please contact scholarworks@uno.edu. 


\title{
A NUMERICAL METHOD FOR COMPUTING RADIALLY SYMMETRIC SOLUTIONS OF A DISSIPATIVE NONLINEAR MODIFIED KLEIN-GORDON EQUATION
}

\section{A Thesis}

Submitted to the Graduate Faculty of the

University of New Orleans

in partial fulfillment of the

requirements for the degree of

\author{
Master of Science \\ in
}

The Department of Physics

by

Jorge Eduardo Macías Díaz

B.S. Universidad Autonoma de Aguascalientes, 1994

M.S. Tulane University Of Louisiana, 1998

Ph.D. Tulane University Of Louisiana, 2001

August 2004 
(C) Copyright by Jorge Eduardo Macías Díaz, 2004

All Rights Reserved 
To Iliana,

Sieg, Carlos, and Néstor with all my love 


\section{Acknowledgments}

First of all I wish to express my gratitude to the University of New Orleans and its Department of Physics for giving me the chance to take graduate studies in applied physics and financially supporting me during the two years I was a graduate student. Being part of this institution was an invaluable academic and personal experience that I will cherish for a lifetime. Academically, the University of New Orleans widen my vision toward branches of mathematics I had never been exposed before; personally, it offered me the chance of interacting with all the great and enjoyable people in the Department of Physics, from secretaries to chairmen.

I also want to thank my thesis adviser, Dr. Ashok Puri, for all his patience and encouragement when every progress seemed to be stuck. Without his knowledge in my area of investigation and his paternal guidance this work would have never been concreted.

Also I wish to thank Iliana, my wife and girlfriend, for her profound devotion and encouragement to complete this paper. Truly, I am eternally indebt for all her support from the beginning of my graduate studies at the University of New Orleans to the realization of this thesis. Hopefully all her support and trust in me will translate in a better future for us and our kids.

Last but not least, I want to thank my sons Sieg, Carlos, and Nestor for their understanding when dad had to work while they played alone in the house. I wished they knew that their innocent and sweet smiles were always source of inspiration for completing this particular episode of my life at the University of New Orleans. I hope that this love they express for papá will never die out in the future! 


\section{Foreword}

In this thesis we label exclusively those formulas which are relevant to our study, that is those expressions that posses a relevant importance on themselves as terminal results or that will be used later on in the derivation of other expressions. These formulas will be invariantly labeled by two numbers presented in the form $(n, m)$, the first corresponds to the chapter where they appear for the first time and the second is the number of labeled formula within that chapter.

Important statements are denominated lemmas, theorems and corollaries. Here we use the standard convention used throughout the mathematical sciences. So, by a lemma we mean a technical result needed to prove a more general statement. It is worth mentioning that a lemma is not important per se, its importance lies in its usefulness to prove further results. By a theorem we understand a statement which is relevant to our investigation and that may answer some of the concerns in this thesis in a rather general form. In this sense a theorem is a terminal result in our research that may or may not need of a lemma to be proved. A corollary is a particular case of a theorem; its importance is manifested in the fact that it answers some specific objectives of this paper. Lemmas, theorems and corollaries are numbered by a single counter within a chapter, and new chapters restart the numeration. Cross-references of lemmas, theorems and corollaries between chapters will be indicated following the same convention as for formulas. Results in Chapter 2 are used in our research, however we do not refer to them in later chapters since they are supposed to be part of the standard literature.

We have included an appendix with the numeric routines we used in our investigation as well as an alphabetic index at the end of this thesis for the sake of easiness in the reading. An index is also included to facilitate the search of concepts in this paper.

The present document was typeset using the packages $\operatorname{LTEX} 2_{\varepsilon}, \mathcal{A M}_{\mathcal{S}}$ ${ }_{\mathrm{AT}} \mathrm{TX}$, and BIBTEX that belong to the mathematical typesetting system MiKTEX version 2.4. $\mathcal{A} \mathcal{M S}$-LTEX was used to create special fonts in our formulas, while BiBTEX was employed to generate the bibliography at the end of the present work. The graphs were generated using computer programs designed to run in Matlab Student Version 6.1.0.450 release 12.1. 


\section{Contents}

List of figures viii

List of tables $\quad x$

Abstract $\quad$ xi

1 Introduction 1

2 Preliminaries $\quad 3$

2.1 Basic definitions . . . . . . . . . . . . . . . . . 3

2.2 Important partial differential equations f . . . . . . . . . . 5

2.3 Modified Klein-Gordon equations . . . . . . . . . . . . . . . . 8

2.3.1 Josephson transmission lines . . . . . . . . . . . . . . . . 9

2.3.2 The statistical mechanics of kinks . . . . . . . . . . . . . . 10

2.3.3 The wave equation revisited . . . . . . . . . . . . . 11

2.4 Elements of numerical analysis . . . . . . . . . . . . . . . . 11

2.4.1 Normed linear spaces . . . . . . . . . . . . . . . . . 11

2.4 .2 Analysis of Stability . . . . . . . . . . . . . . . 12

3 The nonlinear Klein-Gordon equation $\quad \mathbf{1 5}$

3.1 Finite-difference scheme . . . . . . . . . . . . . . . . . 15

3.2 Energy analysis . . . . . . . . . . . . . . . . . . . . . . . 18

3.2.1 Derivation of the energy equation . . . . . . . . . . 18

3.2 .2 Continuous energy . . . . . . . . . . . . . . . . . . 20

3.2.3 Discrete energy . . . . . . . . . . . . . . . . . . . 22

3.3 Numerical results . . . . . . . . . . . . . . . . . . . . . 23

3.4 Discussion . . . . . . . . . . . . . . . . . . . . . . . . 27

4 A modified nonlinear Klein-Gordon equation $\quad 29$

4.1 Finite-difference scheme . . . . . . . . . . . . . . . . . 29

4.1.1 Equivalent formulations . . . . . . . . . . . . . . . . . 29

4.1.2 The externally damped formulation . . . . . . . . . . . . . 31

4.1.3 The externally undamped formulation . . . . . . . . . . . 36

4.1.4 Numerical properties . . . . . . . . . . . . . . . . . . . 37

4.2 Energy analysis . . . . . . . . . . . . . . . . . . . . . 40

4.2.1 Continuous energy . . . . . . . . . . . . . . . . . 40

4.2 .2 Discrete energy . . . . . . . . . . . . . . . . . . . . . . . . . . . . . . 42

4.3 Numerical results . . . . . . . . . . . . . . . . . . . . . . . . . . . . . 44

4.3.1 External damping . . . . . . . . . . . . . . . . . 44 
4.3 .2 Internal damping . . . . . . . . . . . . . . . . . 50

4.4 Discussion . . . . . . . . . . . . . . . . . 58

$\begin{array}{ll}\text { Appendix } & 60\end{array}$

$\begin{array}{ll}\text { References } & 65\end{array}$

$\begin{array}{ll}\text { Vita } & 70\end{array}$ 


\section{List of Figures}

2.1 Schematic representation of a long Josephson junction. . . . . . . . . 9

3.1 Forward-difference scheme for the nonlinear Klein-Gordon equation. .

3.2 Approximate radial solutions at successive times of the nonlinear KleinGordon equation with $G^{\prime}(u)=u^{7}$, initial data $\phi(r)=h(r), \psi(r)=$ $h^{\prime}(r)+h(r) / r$, and boundary condition $u(0.4, t)=0$. . . . . .

3.3 Approximate radial solutions for the given function $G^{\prime}(u)$ at time $t=0.2$ for initial data $\phi(r)=0$ and $\psi(r)=100 h(r)$, and boundary condition $u(0.4, t)=0$.

3.4 Approximate radial solutions for the given function $G^{\prime}(u)$ at time $t=$ 0.2 for initial data $\phi(r)=h(r), \psi(r)=0$ on the left column, and initial conditions $(3.8)$ on the right column. . . . . . . . . . . .

4.1 Forward-difference scheme for the nonlinear Klein-Gordon equation with internal damping. . . . . . . . . . . . . . . . . .

4.2 Approximate radial solutions at successive times of the undamped (solid line) and the damped (dash-dotted line) nonlinear Klein-Gordon equations with $\gamma=0.5, G^{\prime}(u)=u^{7}$, initial data $\phi(r)=h(r), \psi(r)=$ $h^{\prime}(r)+h(r) / r$, and boundary condition $u(0.4, t)=0 . \ldots .$.

4.3 Approximate radial solutions at successive times of the undamped (solid line) and the damped nonlinear Klein-Gordon equation with $\gamma=$ 5 (dashed line) and $\gamma=10$ (dotted line), nonlinear term $G^{\prime}(u)=u^{7}$, initial data $\phi(r)=h(r), \psi(r)=h^{\prime}(r)+h(r) / r$, and boundary condition $u(0.4, t)=0 \ldots \ldots \ldots \ldots \ldots \ldots$

4.4 Approximate radial solutions for the damped nonlinear Klein-Gordon equation with nonlinear function $G^{\prime}(u)$ at time $t=0.2$, for initial data $\phi(r)=0$ and $\psi(r)=100 h(r)$, boundary condition $u(0.4, t)=0$, and damping coefficients $\gamma=0$ (solid line), $\gamma=5$ (dashed line), and $\gamma=10$ (dotted line), respectively. . . . . . . . . . . . . . .

4.5 Approximate radial solutions at successive times of the undamped (solid) and the damped nonlinear Klein-Gordon equation with $\gamma=-1$ (dashed), $\gamma=-5$ (dash-dotted), and $\gamma=-10$ (dotted), nonlinear term $G^{\prime}(u)=u^{7}$, and initial data $\phi(r)=h(r), \psi(r)=h^{\prime}(r)+h(r) / r$, and boundary condition $u(0.4, t)=0 \ldots \ldots \ldots$

4.6 Approximate radial solutions at successive times of the undamped (solid line) and the damped (dash-dotted line) nonlinear Klein-Gordon equations with $\beta=0.0001, G^{\prime}(u)=u^{7}$, initial and boundary conditions $\phi(r)=h(r)$ and $\psi(r)=h^{\prime}(r)+h(r) / r$, and $u(0.4, t)=0$, respectively. 
4.7 Approximate radial solutions at successive times of the undamped (solid) and the damped nonlinear Klein-Gordon equation with $\beta=$ 0.0001 (dashed), $\beta=0.001$ (dash-dotted) and $\beta=0.005$, nonlinear term $G^{\prime}(u)=u^{7}$, initial data $\phi(r)=h(r)$ and $\psi(r)=h^{\prime}(r)+h(r) / r$, and boundary condition $u(0.4, t)=0 \ldots \ldots \ldots$

4.8 Approximate radial solutions for the Klein-Gordon equation with nonlinearity $G^{\prime}(u)$ at $t=0.2$ for initial data $\phi(r)=0, \psi(r)=100 h(r)$ (left column) and $\phi(r)=h(r), \psi(r)=0$ (right column), boundary condition $u(0.4, t)=0$, and $\beta=0$ (solid), $\beta=0.001$ (dashed), and $\beta=0.003$ (dotted). . . . . . . . . . . . . . . .

4.9 Approximate radial solutions for the Klein-Gordon equation with nonlinearity $G^{\prime}(u)$ and $\gamma=5$ at time $t=0.2$, for initial data $\phi(r)=0$ and $\psi(r)=100 h(r)$, boundary condition $u(0.4, t)=0$, and damping coefficients $\beta=0$ (solid line), $\beta=0.0006$ (dashed line), and $\beta=0.005$ (dotted line). . . . . . . . . . . . . . . . . .

4.10 Approximate value of the solution to the Klein-Gordon equation near 0 vs. $\beta$ at $t=0.2$, for nonlinearity $G^{\prime}$ as indicated, $\gamma=0$ (solid), $\gamma=5$ (dashed) and $\gamma=10$ (dotted), initial data $\phi(r)=h(r), \psi(r)=$ $h^{\prime}(r)+h(r) / r$ (left column), and $\phi(r)=0$ and $\psi(r)=100 h(r)$ (right column). . . . . . . . . . . . . . . . . . .

4.11 Approximate radial solutions for the Klein-Gordon equation with nonlinearity $G^{\prime}(u)=u^{5}$ at time $t=0.2$, for initial data $\phi(r)=0$ and $\psi(r)=100 h(r)$ (solid line), boundary condition $u(0.4, t)=0$, and the perturbation prescribed (dotted line). . . . . . . . . . 


\section{List of Tables}

3.1 Maximum amplitudes over space and time of solutions of the nonlinear Klein-Gordon equation for six nonlinear terms $G^{\prime}(u)$. . . . . . . .

4.1 Coefficients $\lambda_{k i}$ in the difference of central second differences approximating the internal damping term of the nonlinear Klein-Gordon equation. . . . . . . . . . . . . . . . .

4.2 Table of relative differences of solutions of the damped nonlinear KleinGordon equation (nonlinear term $G^{\prime}(w)=w^{7}$ and varying damping coefficient $\gamma$ ) with respect to the corresponding undamped solution at different time steps, using $\Delta r=\Delta t=0.002$ and initial data $\phi(r)=$ $h(r), \psi(r)=h^{\prime}(r)+h(r) / r \ldots \ldots \ldots \ldots$

4.3 Table of relative differences of solutions of the damped nonlinear KleinGordon equation (varying nonlinear term $G^{\prime}(w)$ and varying damping coefficient $\gamma$ ) with respect to the corresponding undamped solution at time $t=0.2$, using $\Delta r=\Delta t=0.002$ and common initial data. . .

4.4 Table of relative differences of solutions of the damped nonlinear KleinGordon equation (nonlinear term $G^{\prime}(w)=w^{7}$ and varying damping coefficient $\beta$ ) with respect to the corresponding undamped solution at different time steps, using $\Delta r=\Delta t=0.002$ and initial data $\phi(r)=$ $h(r), \psi(r)=h^{\prime}(r)+h(r) / r \ldots \ldots \ldots \ldots$ 


\section{Abstract}

In this paper we develop a finite-difference scheme to approximate radially symmetric solutions of the initial-value problem

$$
\begin{gathered}
\frac{\partial^{2} w}{\partial t^{2}}-\nabla^{2} w-\beta \frac{\partial}{\partial t}\left(\nabla^{2} w\right)+\gamma \frac{\partial w}{\partial t}+m^{2} w+G^{\prime}(w)=0, \\
\text { subject to : } \begin{cases}w(\bar{x}, 0)=\phi(\bar{x}), & \bar{x} \in D, \\
\frac{\partial w}{\partial t}(\bar{x}, 0)=\psi(\bar{x}), & \bar{x} \in D\end{cases}
\end{gathered}
$$

in an open sphere $D$ around the origin, where the internal and external damping coefficients - $\beta$ and $\gamma$, respectively - are constant, and the nonlinear term has the form $G^{\prime}(w)=w^{p}$, with $p>1$ an odd number. We prove that our scheme is consistent order $\mathcal{O}\left(\Delta t^{2}\right)+\mathcal{O}\left(\Delta r^{2}\right)$ for $G^{\prime}$ identically equal to zero, and provide a necessary condition for it to be stable order $n$. Part of our study will be devoted to compare the physical effects of $\beta$ and $\gamma$. 


\section{Chapter 1}

\section{Introduction}

Klein-Gordon-like equations appear in several branches of modern physics. A modified sine-Gordon equation appears for instance in the study of long Josephson junctions between superconductors when dissipative effects are taken into account [1]. A similar partial differential equation with different nonlinear term appears in the study of fluxons in Josephson tramsmission lines [2]. A modified Klein-Gordon equation appears in the statistical mechanics of nonlinear coherent structures such as solitary waves in the form of a Langevin equation [3]; here no internal damping coefficient appears, though. Finally, our differential equation describes the motion of a string with internal and external damping in a non-Hookean medium.

The classical $(1+1)$-dimensional linear Klein-Gordon equation has an exact soliton-like solution in the form of a traveling wave [4]. Some results concerning the analytic behavior of solutions of nonlinear Klein-Gordon equations have been established [5, 6, 7]; however, no exact method of solution is known for arbitrary initial-value problems involving this equation. From that point of view it is important to investigate numerical techniques to describe the evolution of radially symmetric solutions of our problem.

It is worth mentioning that some numerical research has been done in this direction. Strauss and Vázquez [8] developed a finite-difference scheme to approximate radially symmetric solutions of the nonlinear Klein-Gordon equation for the same nonlinear term we study in this paper; one of the most important features of their numerical method was that the discrete energy associated with the differential equation is conserved. The numerical study of the sine-Gordon model that describes the Josephson tunnel junctions has been undertaken by Lomdahl et al. [2]. Numerical simulations have also been performed to solve the $(1+1)$-dimensional Langevin equation [9].

In this paper we present a numerical analysis of the radially symmetric solutions to a modified nonlinear Klein-Gordon equation that generalizes the applications described in the previous paragraphs, as well as several other well-known physically and biologically important partial differential equations.

$$
* * *
$$

Chapter 2 provides a list of important second-order partial differential equations that constitute particular cases of the equation under study. We state the general form of our problem and describe the most important applications that it models. 
We close Chapter 2 stating some important definitions and results from numerical analysis that we use in this thesis without reference.

The next chapter is devoted to the study of our modified nonlinear KleinGordon equation without damping. We show that the energy associated with our differential equation is constant throughout time and derive a conditionally stable finite-difference scheme consistent to the second order, which has the property that the discrete energy is conserved. We close that chapter showing numerical results that are in agreement with [8].

In the last chapter we derive a numerical method to approximate radially symmetric solutions of the modified nonlinear Klein-Gordon equation under study. As we will observe, if no external damping is present then the inclusion of an extra term in the finite-difference scheme of Chapter 3 yields a second-order approximation to the exact solution of our problem. For the externally damped case we notice that the solution of the problem requires a more complicated reformulation in order to achieve consistency to the second order. We analyze the energy associated with our equation and derive a discrete expression for the rate of change of energy. We study the stability and consistency of our numerical methods in detail and show graphically that our results are in agreement with the non-dissipative case.

The appendix at the end of this work contains the most basic form of the numerical routines we developed to approximate the solutions of our equation. The programs do not contain the subroutines to generate the graphs included in this thesis. 


\section{Chapter 2}

\section{Preliminaries}

The nonlinear Klein-Gordon equation is one of the most important and simplest nonlinear differential equations that appear in relativistic quantum mechanics. As a second-order partial differential equation, the Klein-Gordon equation generalizes several other important problems in various branches of physics, chemistry and mathematical biology that range from the classical diffusion equation to the stochastic Fisher-KPP equation, from the classical wave equation to the Schrödinger and the telegrapher's equations.

The present chapter is devoted to introduce and evidence the importance of the differential equation under study in this thesis. We also present some important definitions and results of numerical analysis that will be used without reference in further chapters.

\subsection{Basic definitions}

By a domain we understand a closed connected subset of $\mathbb{R}^{n}$. A function $u$ defined in a domain $D$ is said to be have compact support if it is zero outside a compact subset of $D$.

A function $u$ defined on a domain $D$ is called smooth in $D$ if it has continuous partial derivatives of all orders in $D$. The function $u$ is called small at infinity if for every $\bar{x}_{0}$ in the boundary of $D$,

$$
\begin{aligned}
& \lim _{\substack{x \rightarrow \bar{x}_{0} \\
\bar{x} \in D}} u(\bar{x})=0 .
\end{aligned}
$$

Let $a, b, c, d$ and $e$ be real numbers with at least one of $a, b$ or $c$ not equal to zero. A second-order partial differential equation in the variables $x$ and $y$ with constant coefficients is an equation of the form

$$
a \frac{\partial^{2} u}{\partial x^{2}}+b \frac{\partial^{2} u}{\partial x \partial y}+c \frac{\partial^{2} u}{\partial y^{2}}+d \frac{\partial u}{\partial x}+e \frac{\partial u}{\partial y}=F(x, y)
$$

where $u$ is a function of $(x, y)$ usually assumed to be defined and of compact support in some domain $D$, that has continuous partial derivatives up to the second order in 
$D$. The number $b^{2}-4 a c$ is called the discriminant of Equation (2.1) and yields a criterion to classify second-order partial differential equations:

- If $b^{2}-4 a c>0$ then Equation (2.1) is called a hyperbolic equation. As an example of this type of equation we have the classical one-dimensional wave equation

$$
\frac{\partial^{2} u}{\partial x^{2}}=\frac{1}{\nu^{2}} \frac{\partial^{2} u}{\partial t^{2}}
$$

It describes the vertical disturbance of a wave with phase velocity $\nu$ as it travels on the horizontal direction. The wave equation applies to a stretched string or a plane electromagnetic wave. Given initial and boundary conditions the wave equation can be solved exactly by using a Fourier transform method or via separation of variables.

- If $b^{2}-4 a c=0$ then Equation (2.1) is called a parabolic equation. An example of parabolic equation is the one-dimensional diffusion equation (also called heat equation)

$$
\frac{\partial u}{\partial t}=\kappa \frac{\partial^{2} u}{\partial x^{2}}
$$

This equation commonly arises in problems of heat conductivity. In those situations $\kappa$ represents thermal diffusivity and $u$ represents temperature. If initial and boundary conditions are given, the diffusion equation can be solved analytically by separation of variables.

- If $b^{2}-4 a c<0$ then Equation (2.1) is called an elliptic equation. Laplace's equation

$$
\frac{\partial^{2} u}{\partial x^{2}}+\frac{\partial^{2} u}{\partial y^{2}}=0
$$

is an example of an elliptic equation. It is satisfied by the potential of any distribution of matter which attracts according to the Newtonian Law. A solution to Laplace's equation is uniquely determined if the value of the function or the normal derivative of the function is specified on all boundaries.

We must remark that the wave equation, the heat equation and Laplace's equation have generalizations that model the corresponding physical phenomena in three dimensions. For example, the wave equation in three space variables reads

$$
\nabla^{2} u=\frac{1}{\nu^{2}} \frac{\partial^{2} u}{\partial t^{2}}
$$

where $u$ is a scalar function that depends on the space coordinate $(x, y, z)$ and time $t$. The symbol $\nabla^{2}$ denotes the Laplacian differential operator, which is the divergence of the gradient of a scalar function. With this notation the three-dimensional diffusion equation is described by the equation

$$
\frac{\partial u}{\partial t}=\frac{1}{\kappa} \nabla^{2} u
$$


and the three-dimensional Laplace's equation by

$$
\nabla^{2} u=0
$$

Let $V$ and $\rho$ be scalar functions depending only on space. An important variation of the three-dimensional Laplace's equation occurs in classical electromagnetic theory when relating the electric potential $V$ of a distribution and its charge density $\rho$. The relation between $V$ and $\rho$ is described by the equation $\epsilon_{0} \nabla^{2} V=\rho$, which is called Poisson's equation. More generally, every equation of the form

$$
\nabla^{2} u=F(x, y, z, t)
$$

where $u$ is a scalar function depending on $x, y, z$ and $t$, is called a Poisson equation.

Another useful classification of second-order partial differential equations with constant coefficients is in terms of a property called linearity. Differential equation (2.1) is called linear if for arbitrary real constants $k_{1}, k_{2}$ and solutions $u_{1}, u_{2}$ of (2.1), $k_{1} u_{1}+k_{2} u_{2}$ is also a solution of (2.1).

Finally, if the variable time is one of the independent variables of the scalar function $u$ then the term $k \partial u / \partial t$ in the differential equation modeling $u$ is called the external damping term and the constant $k$ is called the external damping coefficient. The differential equation is said to be damped if $k$ is not equal to zero, otherwise it is called undamped.

\subsection{Important partial differential equations}

Many other three-dimensional generalizations of the wave equation, the diffusion equation and Laplace's equation happen to appear in mathematical physics and biology. For example, the manipulation of Maxwell's equations to obtain propagating waves gives rise to the so called Helmholtz equation [10], whose general form is

$$
\nabla^{2} u+k^{2} u=0
$$

where $k$ is a real constant and $u$ is a scalar function in the variables $x, y, z, t$. Obviously, Helmholtz equation is a linear second-order partial differential equation that generalizes the three-dimensional wave equation.

Another physical example appears in the field of non-relativistic quantum mechanics: Let $\hbar$ denote Planck's original constant divided by $2 \pi$. The wave function associated to a particle of mass $m$ with potential scalar function $V$ is a scalar function $u$ that depends on the position vector $(x, y, z)$ of the particle and the time $t$, given by the differential equation

$$
i \hbar \frac{\partial u}{\partial t}=-\frac{\hbar^{2}}{2 m} \nabla^{2} u+V u .
$$

This differential equation is called Schrödinger's equation. In this equation the scalar function $u$ may be complex, but the square of its modulus is a real scalar function that represents the probability density function associated with the location of 
the particle at any time. It is worth noticing that Schrödinger's equation provides a mathematical generalization of the three-dimensional diffusion equation. Observe that because the scalar function $V$ does not need to be constant, Schrödinger's equation is a linear partial differential equation with not necessarily constant coefficients.

The relativistic counterpart of Schrödinger's equation is the Klein-Gordon equation. By the linear Klein-Gordon equation we understand the linear secondorder partial differential equation

$$
\nabla^{2} u=\frac{1}{c^{2}} \frac{\partial^{2} u}{\partial t^{2}}+m^{2} u
$$

where $m$ is a real constant and $u$ is a scalar function of position and time. This is the equation for a relativistic quantum-mechanical scalar (spin-zero) particle of mass $m$. The exact solution of this equation in the form of a traveling wave is given in [4]. An important nonlinear variation of this equation that often appears in the study of the collisional properties of solitons $[11,12]$ and a number of other physical applications $[7,13,14]$ is the sine-Gordon equation

$$
\nabla^{2} u=\frac{1}{c^{2}} \frac{\partial^{2} u}{\partial t^{2}}+m^{2} \sin u
$$

In mathematical biology, consider a population distributed in a linear habitat with uniform density. If at any point of the habitat a mutation advantageous to survival occurs then the mutant gene increases at the expense of the allelomorphs previously occupying the same locus. Mathematically, let $u$ be the frequency of the mutant gene and let $m$ be a constant representing intensity of selection in favor of the mutant gene. Then $u$ must satisfy Fisher's equation (also called the Fisher-KPP equation)

$$
\frac{\partial u}{\partial t}=k \frac{\partial^{2} u}{\partial x^{2}}+F(u)
$$

where $k$ is a diffusion coefficient and $u$ depends on the position $x$ in the linear habitat and time $t$ given in generations. This parabolic equation was simultaneously and independently investigated by Fisher [15] and Kolmogoroff et al. [16], using $F(u)=$ $m u(1-u)$. It is used also in describing the process of epidermal wound healing [17]. Other applications appear in the theory of superconducting electrodynamics [18] and in the study of excitons [19]. Fisher's equation is a nonlinear equation that obviously generalizes the three-dimensional diffusion model if we rewrite Fisher's equation as

$$
\frac{\partial u}{\partial t}=k \nabla^{2} u+F(u)
$$

The stochastic Fisher-KPP equation is the one-dimensional Fisher equation with $F(u)=m u(1-u)+\gamma \sqrt{u(1-u)} \eta(x, t)$, where $0 \leq u \leq 1, \gamma$ is a real constant, and $\eta(x, t)$ is a Gaussian white noise process in space and time with mean equal to zero [20]. To fix ideas, we may think of a noise as a random signal of known statistical properties of amplitude, distribution, and spectral density. A noise is a white noise in space and time if it is uncorrelated in these two variables, and it is Gaussian if its probability density function over a given frequency band is nor- 
mal. The stochastic Fisher-KPP equation is a stochastic partial differential equation that describes random walk processes that have applications in hydrodynamics and economics.

Second-order partial differential equations describing diffusion or conduction happen to appear in the area of thermodynamics [21]. Heat conduction is understood as the transfer of heat from warm areas to cooler ones, and effectively occurs by diffusion. Under the assumption of a macroscopic continuum formulation, the Fourier equation [22] for the heat flux $\bar{q}$ in a medium of density $\rho$, mass heat capacity $C_{P}$, and temperature function $u$, is

$$
\bar{q}=-k \nabla u
$$

where both $\bar{q}$ and $u$ depend on the three spatial coordinates and time, $k=\rho \kappa C_{P}$ is the thermal conductivity of the medium, and $\kappa$ is the thermal diffusivity term of the classical diffusion equation.

The previously mentioned Fourier heat conduction equation is diffusive and does not account for the temperature propagation speed in transient situations. Because of certain issues argued and identified earlier, attempts to account for a finite speed of heat propagation have evolved over the years. The Maxwell-Cattaneo model [23], which is based on the notion of relaxing the heat flux, is given as

$$
\tau \frac{\partial \bar{q}}{\partial t}=-\bar{q}-k \nabla u
$$

where $\tau$ is the relaxation time. Assuming that there are no heat sources and that $k$ is constant, the one-dimensional version of the Maxwell-Cattaneo equation together with the energy equation

$$
\rho C_{P} \frac{\partial u}{\partial t}+\frac{\partial q}{\partial x}=0
$$

yield the hyperbolic equation

$$
\tau \rho C_{P} \frac{\partial^{2} u}{\partial t^{2}}-k \frac{\partial^{2} u}{\partial x^{2}}+\rho C_{P} \frac{\partial u}{\partial t}=0 .
$$

Obviously, it can be generalized to the three-dimensional case as

$$
\frac{\partial^{2} u}{\partial t^{2}}-\frac{k}{\tau \rho C_{P}} \nabla^{2} u+\frac{1}{\tau} \frac{\partial u}{\partial t}=0
$$

The telegraph equation is a hyperbolic equation that describes heat or mass transport. It models phenomena that are mixtures between diffusion and wave propagation. In this model a small section of a telegraph wire is treated to study the pulse of voltage moving along the wire. It was studied in 1876 by Heaviside in his research on coaxial marine telegraph cables [24]. The telegraph equation is the linear second-order partial differential equation

$$
\frac{\partial^{2} u}{\partial x^{2}}-\frac{1}{\nu^{2}} \frac{\partial^{2} u}{\partial t^{2}}-\gamma \frac{\partial u}{\partial t}-b^{2} u=0
$$


where $\nu$ is positive, and $\gamma$ and $b$ are nonnegative constants. The one-dimensional wave equation is just a particular case of the telegraph equation with $\gamma$ and $b$ both equal to zero. The generalization of the telegraph equation to three dimensions is

$$
\nabla^{2} u-\frac{1}{\nu^{2}} \frac{\partial^{2} u}{\partial t^{2}}-\gamma \frac{\partial u}{\partial t}-b^{2} u=0
$$

\subsection{Modified Klein-Gordon equations}

The objective of this paper is to study a general form of the Klein-Gordon equation that embraces the partial differential equations described in the previous section and, at the same time, takes into account a third-order term proportional to the Laplacian of the partial derivative of $u$ in time, which physically represents the internal damping term. More precisely, let $u$ be a function of the spatial variables $X, Y, Z$, and the time variable $T$. The nonlinear partial differential equation with constant coefficients that we wish to study in this thesis is

$$
a \frac{\partial^{2} u}{\partial T^{2}}-b \nabla^{2} u-c \frac{\partial}{\partial T}\left(\nabla^{2} u\right)+d \frac{\partial u}{\partial T}+m^{2} u+G^{\prime}(u)=0
$$

Let $x=X / \sqrt{b}, y=Y / \sqrt{b}, z=Z / \sqrt{b}$, and $t=T / \sqrt{a}$ for $a$ and $b$ positive numbers. Let $\beta=c /(b \sqrt{a})$ and $\gamma=d / \sqrt{a}$. Our problem can be stated in dimensionless form as

$$
\begin{gathered}
\frac{\partial^{2} u}{\partial t^{2}}-\nabla^{2} u-\beta \frac{\partial}{\partial t}\left(\nabla^{2} u\right)+\gamma \frac{\partial u}{\partial t}+m^{2} u+G^{\prime}(u)=0, \\
\text { subject to : } \begin{cases}u(\bar{x}, 0)=\phi(\bar{x}), & \bar{x} \in D, \\
\frac{\partial u}{\partial t}(\bar{x}, 0)=\psi(\bar{x}), & \bar{x} \in D .\end{cases}
\end{gathered}
$$

This initial-value problem will be referred to as the modified nonlinear Klein-Gordon equation or the dissipative nonlinear Klein-Gordon equation, and its numerical study for the particular choice $G^{\prime}(u)=u^{p}$, for $p>1$ an odd number, is the topic of this paper. We identify the term containing the coefficient $\beta$ as the internal damping term, while the term containing $\gamma$ is easily identified as the external damping term. Needless to say that the differential equation in (2.2) generalizes the equations listed in Section 2.2 either by choosing suitable coefficients or by suppressing terms; the classical Klein-Gordon equation, for instance, can be obtained by setting $\beta$ and $\gamma$ both equal to zero and $G^{\prime}$ identically zero.

The following is the major theoretic result we will use in our investigation. It is valid only for certain classical one-dimensional nonlinear Klein-Gordon equations. Here $M(t)$ represents the amplitude of a solution of (2.2) at time $t$, that is

$$
M(t)=\max _{x}|u(x, t)| .
$$


Theorem 1. Let $\beta$ and $\gamma$ be both equal to zero, and let $G^{\prime}(u)=|u|^{p-1} u$. Suppose that $\phi$ and $\psi$ are smooth and small at infinity. Then

(1) If $p<5$, a unique smooth solution of (2.2) exists with amplitude bounded at all time [6].

(2) If $p \geq 5$, a weak solution exists for all time [25].

(3) For $p>8 / 3$ and for solutions of bounded amplitude, there is a scattering theory; in particular, they decay uniformly as fast as $M(t) \leq c(1+|t|)^{-3 / 2}$ $[26]$.

As we stated in the introductory chapter, initial-value problem (2.2) has applications in several physical problems. We describe now some of them.

\subsubsection{Josephson transmission lines}

A Josephson junction is a type of electronic circuit capable of switching at very high speeds when operated at temperatures approaching absolute zero. Named for the British physicist who designed it, a Josephson junction exploits the phenomenon of superconductivity, that is the ability of certain materials to conduct electric current with practically zero resistance. Josephson junctions are used in certain specialized instruments such as highly-sensitive microwave detectors, magnetometers, and quantum interference devices.

A Josephson junction is made up of two superconductors, separated by a nonsuperconducting layer so thin that electrons can cross through the insulating barrier. The flow of current between the superconductors in the absence of an applied voltage is called a Josephson current, and the movement of electrons across the barrier is known as Josephson tunneling. Two or more junctions joined by superconducting paths form what is called a Josephson interferometer.

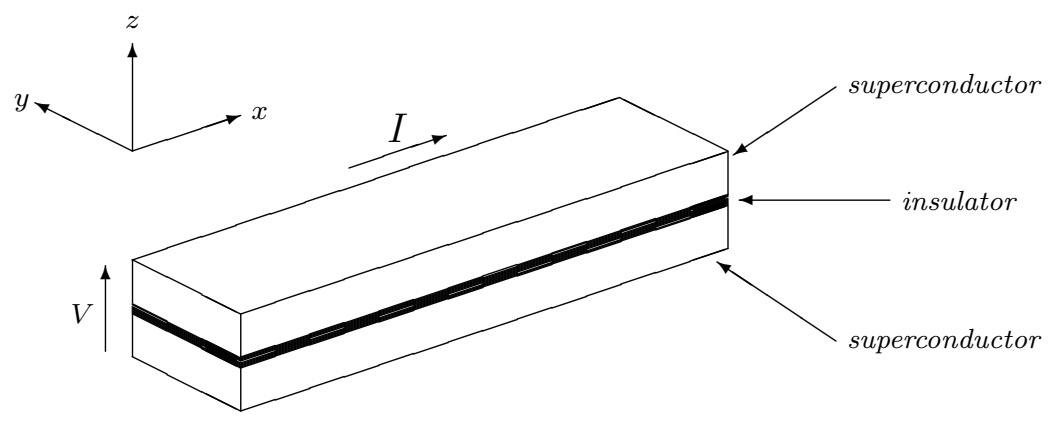

Figure 2.1: Schematic representation of a long Josephson junction.

While researching superconductivity, Josephson studied the properties of a junction between two superconductors [27]. Following up on earlier work by Leo Esaki and Ivar Giaever, he demonstrated that in a situation when there is electron flow between two superconductors through an insulating layer (in the absence of an applied voltage), and a voltage is applied, the current stops flowing and oscillates 
at a high frequency. This phenomenon is called the Josephson effect, and it is influenced by magnetic fields in the vicinity, a capacity that enables the Josephson junction to be used in devices that measure extremely weak magnetic fields, such as superconducting quantum interference devices. For their efforts, Josephson, Esaki, and Giaever shared the Nobel Prize for Physics in 1973.

It is worthwhile mentioning that the theory of low temperature conductivity tells us that a superconductor is a system where a fraction of the conduction electrons form pairs called Cooper pairs. In these pairs the two electrons have opposite momentum and spin. These pairs are able to condense in the same quantum state so that the superconductor can be described by a single macroscopic wave function

$$
\Psi=\sqrt{\rho} e^{i \phi}
$$

Here $\rho$ represents the pair density and $\phi$ is the quantum phase common to all pairs.

For the long Josephson junction, in dealing with real transmission lines one must take into account losses, bias, and junction irregularities which influence motion [1]. When we account for all of these effects, we obtain the third order partial differential equation

$$
\frac{\partial^{2} \phi}{\partial x^{2}}-\frac{\partial^{2} \phi}{\partial t^{2}}-\alpha \frac{\partial \phi}{\partial t}+\beta \frac{\partial^{3} \phi}{\partial x^{2} \partial t}=\sin \phi-\gamma
$$

where $\alpha, \beta$ and $\gamma$ are constants.

\subsubsection{The statistical mechanics of kinks}

The statistical mechanics of kinks (that is, exact solitary waves) of nonlinear coherent structures has been studied by two approaches. In the first approach one assumes that the kinks may be treated as weakly interacting elementary excitations. Provided the kink density is low, the canonical partition function can be found by standard methods $[28,29,30]$. Alternatively, it is possible to calculate the partition function to exploit a transfer operator technique. This method was used by Krumhansl and Schrieffer in [29], and it showed that in the low temperature limit the partition function naturally factorizes into two contributions: A tunneling term which they were able to identify with the kink contribution, and the remainder which they identified as linearized phonons (by a phonon we mean a quantized mode of vibration occurring in a rigid crystal lattice, such as the atomic lattice of a solid).

The ideas of Krumhansl and Schrieffer were further refined and extended to a wider class of systems [30]. In particular, interactions of kinks with linearized phonons were considered, leading to substantial corrections of results.

Computer simulations based on standard methods [9] made possible to verify results on the equilibrium statistical mechanics of kinks using a dimensionless Langevin equation describing the $(1+1)$-dimensional theory:

$$
\frac{\partial^{2} \phi}{\partial t^{2}}=\frac{\partial^{2} \phi}{\partial x^{2}}-\gamma \frac{\partial \phi}{\partial t}-\phi\left(1-\phi^{2}\right)+F(x, t)
$$




\subsubsection{The wave equation revisited}

Initial-value problem (2.2) also describes the mechanical motion of strings for certain physical situations. Consider the one-dimensional motion of a string immersed in a non-Hookean medium. We represent the vertical motion of the string as a function $u(r, t)$ of horizontal position and time, and the nonlinear force of the medium by $G^{\prime}(u)$. The string is assumed to posses internal damping due to its inner stiffness, which is proportional to $u_{r r t}$. Finally, we assume that there exists friction between the string and the medium that derives in a force which opposes the motion of the string and is proportional to the vertical velocity of the string. In these circumstances, the motion of our string will be described by (2.2).

\subsection{Elements of numerical analysis}

In our investigation, we are interested in developing finite-difference schemes to approximate radially symmetric solutions of modified nonlinear Klein-Gordon equations. In order to determine how accurate our approximations are, we need to introduce the notions of convergence, consistency and stability. To understand these concepts we must first clarify some ideas from mathematical analysis. Here we follow [31] and [32]. Throughout $K$ denotes the fields $\mathbb{R}$ and $\mathbb{C}$.

\subsubsection{Normed linear spaces}

A norm on a vector space $V$ over a scalar field $K$ is a function $\|\cdot\|$ that associates every element of $V$ with a real number, such that for any vectors $\bar{u}$ and $\bar{v}$, and any scalar $a$, the following properties are satisfied:

(i) $\quad\|\bar{v}\| \geq 0$, and $\|\bar{v}\|=0$ iff $\bar{v}=0$,

(ii) $\quad\|a \bar{v}\|=|a|\|\bar{v}\|$, and

(iii) $\quad\|\bar{u}+\bar{v}\| \leq\|\bar{u}\|+\|\bar{v}\|$.

It is worthwhile mentioning that a vector space with a norm associated with it is called a normed linear space or simply normed space. The following are examples of normed linear spaces with the given norms.

Example 2. Denote by $|\cdot|$ the standard norm in $K$. The linear space $K^{n}$ can be given the $p$-norm $(p \geq 1)$

$$
\|\bar{x}\|_{p}=\left(\sum_{i=1}^{n}\left|x_{i}\right|^{p}\right)^{1 / p} .
$$

The 1-norm and the 2-norm in $K^{n}$ are called the the taxicab norm and the Euclidean norm , respectively. $K^{n}$ can also be normed by the so called infinity norm $\|\bar{x}\|_{\infty}=\max \left\{\left|x_{1}\right|, \ldots,\left|x_{n}\right|\right\}$. 
ExAmple 3. Let $\Delta x$ and $p$ be positive numbers with $p>1$. The space $\ell_{p, \Delta x}$ is the normed linear space of all infinite sequences $\mathbf{u}=\left(\ldots, u_{-1}, u_{0}, u_{1}, \ldots\right)$ of elements in $K$ with vector addition and scalar multiplication given componentwise, such that $\sum_{-\infty<j<\infty}\left|u_{j}\right|^{p}<\infty$. The norm is given by

$$
\|\mathbf{u}\|_{p, \Delta x}=\left(\sum_{k=-\infty}^{\infty}\left|u_{k}\right|^{p} \Delta x\right)^{1 / p} .
$$

The space $\ell_{p}$ is defined to be the space $\ell_{p, 1}$. If $p$ is equal to 2 then $\ell_{p, \Delta x}$ is called the energy space.

EXAmPLE 4 . Let $\lambda$ represent the Lebesgue measure on $X \subseteq \mathbb{R}$. The space $L_{p}(X)$ for $p>1$ is the normed linear space of all equivalence classes of functions $f: X \rightarrow \mathbb{R}$ under the relation of equivalence almost everywhere, together with addition and scalar multiplication defined in representatives, such that $\int_{X} f^{p} d \lambda<\infty$. Its norm is given by

$$
\|f\|_{p}=\left(\int_{X} f^{p} d \lambda\right)^{1 / p}
$$

EXAmple 5. Let $\|\cdot\|$ be any norm in $K^{n}$. The space of all $n \times n$-matrices with coefficients in $K$ is a normed linear space with the usual operations of addition of matrices and scalar multiplication, with matrix norm defined by

$$
\|Q\|=\sup _{\|\bar{u}\| \leq 1}\{Q \bar{u}\}
$$

\subsubsection{Analysis of Stability}

\section{Convergence}

A difference scheme $L_{k}^{n} u_{k}^{n}=G_{k}^{n}$ approximating the partial differential equation $\mathcal{L} v=F$ is a convergent scheme at time $t$ in the norm $\|\cdot\|$ of $\ell_{p, \Delta x}$ if, as $(n+1) \Delta t \rightarrow t$

$$
\left\|\mathbf{u}^{n+1}-\mathbf{v}^{n+1}\right\| \rightarrow 0
$$

as $\Delta x, \Delta t \rightarrow 0$. Here $\mathbf{u}^{n}=\left(\ldots, u_{-1}^{n}, u_{0}^{n}, u_{1}^{n}, \ldots\right)$ and $\mathbf{v}^{n}=\left(\ldots, v_{-1}^{n}, v_{0}^{n}, v_{1}^{n}, \ldots\right)$ are the sequences representing the vector of approximations to the solution of the partial differential equation and the vector of exact solutions whose $k$-th component is $v(k \Delta x, n \Delta t)$, respectively.

\section{Consistency}

The difference scheme $\mathbf{u}^{n+1}=Q \mathbf{u}^{n}+\Delta t \mathbf{G}^{n}$ is consistent with the partial differential equation $\mathcal{L} v=F$ in the norm $\|\cdot\|$ if the solution $v$ of the differential equation satisfies

$$
\mathbf{v}^{n+1}=Q \mathbf{v}^{n}+\Delta t \mathbf{g}^{n}+\Delta t \tau^{n}
$$


and $\left\|\tau^{n}\right\| \rightarrow 0$ as $\Delta x, \Delta t \rightarrow 0$. Moreover, the scheme is said to be accurate with order $\mathcal{O}\left(\Delta x^{p}\right)+\mathcal{O}\left(\Delta t^{q}\right)$ if

$$
\left\|\tau^{n}\right\|=\mathcal{O}\left(\Delta x^{p}\right)+\mathcal{O}\left(\Delta t^{q}\right)
$$

\section{Stability}

One interpretation of stability of a finite-difference scheme is that, for a stable scheme, small errors in the initial conditions cause small errors in the solution. As we will see, the definition does allow the errors to grow but limits them to grow no faster than exponential. More precisely, the finite-difference scheme $\mathbf{u}^{n+1}=Q \mathbf{u}^{n}$ is said to be stable with respect to the norm $\|\cdot\|$ if there exist positive constants $\Delta x_{0}$ and $\Delta t_{0}$, and nonnegative constants $K$ and $\beta$ so that

$$
\left\|\mathbf{u}^{n+1}\right\| \leq K e^{\beta t}\left\|\mathbf{u}^{0}\right\|
$$

for $0 \leq t=(n+1) \Delta t, 0<\Delta x \leq \Delta x_{0}$ and $0<\Delta t \leq \Delta t_{0}$. If further restrictions on the relationship between $\Delta t$ and $\Delta x$ are needed in order to guarantee stability of the finite-difference scheme, we say that the scheme is conditionally stable.

One characterization of stability that is often useful comes from the inequality in the definition above. We state this in the following result.

TheOREM 6. The scheme $\mathbf{u}^{n+1}=Q \mathbf{u}^{n}$ is stable with respect to the norm $\|\cdot\|$ if and only if there exist positive constants $\Delta x_{0}$ and $\Delta t_{0}$, and nonnegative constants $K$ and $\beta$ so that

$$
\left\|Q^{n+1}\right\| \leq K e^{\beta t}
$$

for $0 \leq t=(n+1) \Delta t, 0<\Delta x \leq \Delta x_{0}$ and $0<\Delta t \leq \Delta t_{0}$.

The difference scheme $\mathbf{u}^{n+1}=Q \mathbf{u}^{n}$ is said to be stable order $n$ with respect to the norm $\|\cdot\|$ if there exist positive constants $\Delta x_{0}$ and $\Delta t_{0}$, and nonnegative constants $K_{1}, K_{2}$ and $\beta$ such that

$$
\left\|\mathbf{u}^{n+1}\right\| \leq\left(K_{1}+n K_{2}\right) e^{\beta t}\left\|\mathbf{u}^{0}\right\|,
$$

for $0 \leq t=(n+1) \Delta t, 0<\Delta x \leq \Delta x_{0}$ and $0<\Delta t \leq \Delta t_{0}$. Obviously, if a finitedifference scheme is stable then it will be stable order $n$. We also realize that the above definition is equivalent to requiring that $Q$ satisfy $\left\|Q^{n}\right\| \leq\left(K_{1}+n K_{2}\right) e^{\beta t}$.

The use of the discrete Fourier transform is a useful tool in the analysis of stability of finite-difference schemes for initial-value problems. We define the discrete Fourier transform of $\mathbf{u} \in \ell_{2}$ as the function $\hat{u} \in L_{2}([-\pi, \pi])$ given by

$$
\hat{u}(\xi)=\frac{1}{\sqrt{2 \pi}} \sum_{m=-\infty}^{\infty} e^{-i m \xi} u_{m}
$$

for $\xi \in[-\pi, \pi]$. The $\ell_{2}$ vectors that we will be using later will be the $\ell_{2, \Delta x}$ vectors that are the solutions to our finite-difference schemes at time step $n$.

EXAMPLE 7. The central second-order difference is the linear operator $\delta^{2}$ that associates with each infinite sequence $\mathbf{u}=\left(\ldots, u_{-1}, u_{0}, u_{1}, \ldots\right)$ of real numbers the 
infinite sequence $\delta^{2} \mathbf{u}$ whose $m$-th component is given by $u_{m+1}-2 u_{m}+u_{m-1}$. It is easy to check that the Fourier transform of $\delta^{2} \mathbf{u}$ is given by $-4 \sin ^{2} \frac{\xi}{2} \hat{u}$.

It is important to remark that if $\mathbf{u} \in \ell_{2}$ has discrete Fourier transform $\hat{u}$ then $\|\hat{u}\|_{2}=\|\mathbf{u}\|_{2}$, where the first norm is the $L_{2}$-norm on $[-\pi, \pi]$ and the second norm is the $\ell_{2}$-norm. This fact constitutes a bridge between the spaces $\ell_{2}$ and $L_{2}([-\pi, \pi])$ that provides us with the following important result for stability.

THEOREM 8. The sequence $\left\{\mathbf{u}^{n}\right\}$ is stable in $\ell_{2, \Delta x}$ if and only if the sequence $\left\{\hat{u}^{n}\right\}$ is stable in $L_{2}([-\pi, \pi])$.

Let $\mathbf{u}^{n+1}=Q \mathbf{u}^{n}$ be a finite difference scheme. Taking discrete Fourier transform in both sides we obtain an equation of the form $\hat{u}^{n+1}=A(\xi) \hat{u}^{n}$. The matrix $A(\xi)$ is called the amplification matrix of the difference scheme. By virtue of Theorem 8, the stability of the scheme depends on the growth of the amplification matrix raised to the $n$-th power.

TheOREM 9 (LAX TheOREM). If a two-level difference scheme $\mathbf{u}^{n+1}=Q \mathbf{u}^{n}+\Delta t \mathbf{G}^{n}$ is consistent in the norm $\|\cdot\|$ to an initial-value problem and is stable with respect to $\|\cdot\|$, the it is convergent with respect to $\|\cdot\|$. 


\section{Chapter 3}

\section{The nonlinear Klein-Gordon equation}

In the present chapter we describe a numerical method to approximate radially symmetric solutions of the nonlinear Klein-Gordon equation. The energy analysis of this equation shows that the total energy is conserved. We propose a discrete scheme for the energy of this equation and prove that it is conserved at every time step. Finally, we show that our results are in general agreement with Strauss and Vázquez [8].

\subsection{Finite-difference scheme}

Let $u$ be a function of position $\bar{x}$ and time $t$, and suppose that $\phi$ and $\psi$ are functions of $\bar{x}$ of compact support in a domain $D$ of $\mathbb{R}^{3}$ with continuous partial derivatives up to the second order in $D$. The problem under study in this chapter can be rewritten in dimensionless form as the initial-value problem

$$
\begin{gathered}
\frac{\partial^{2} u}{\partial t^{2}}-\nabla^{2} u+m^{2} u+G^{\prime}(u)=0, \\
\text { subject to : } \begin{cases}u(\bar{x}, 0)=\phi(\bar{x}), & \bar{x} \in D, \\
\frac{\partial u}{\partial t}(\bar{x}, 0)=\psi(\bar{x}), & \bar{x} \in D .\end{cases}
\end{gathered}
$$

We are interested in computing radially symmetric solutions of (3.1), that is solutions of the form $u=u(r, t)$ with $r=\|\bar{x}\|$, where $\|\cdot\|$ denotes the usual Euclidean norm in $\mathbb{R}^{3}$. For the sake of simplicity we may assume that $D$ represents the open sphere with center in the origin and radius $L$, and that $\phi$ and $\psi$ are radially symmetric in $D$ and small at infinity.

In spherical coordinates

$$
r \nabla^{2} u=r \frac{\partial^{2} u}{\partial r^{2}}+2 \frac{\partial u}{\partial r}
$$

so that our nonlinear Klein-Gordon equation reads 


$$
\frac{\partial^{2} u}{\partial t^{2}}-\frac{\partial^{2} u}{\partial r^{2}}-\frac{2}{r} \frac{\partial u}{\partial r}+m^{2} u+G^{\prime}(u)=0, \quad \text { for } 0<r<L .
$$

In order to simplify this equation we let $v(r, t)=r u(r, t)$. Obviously $v$ satisfies $v(0, t)=0$, for every $t \geq 0$. Moveover, for every $0<r<L$

$$
\begin{array}{ll}
\frac{1}{r} \frac{\partial^{2} v}{\partial t^{2}} & =\frac{\partial^{2} u}{\partial t^{2}}, \\
\frac{1}{r} \frac{\partial^{2} v}{\partial r^{2}} & =\frac{\partial^{2} u}{\partial r^{2}}+\frac{2}{r} \frac{\partial u}{\partial r}
\end{array}
$$

These equations together with the initial conditions in (3.1) and the boundary condition stated in the previous paragraph yield the mixed-value problem

$$
\begin{aligned}
& \frac{\partial^{2} v}{\partial t^{2}}-\frac{\partial^{2} v}{\partial r^{2}}+m^{2} v+r G^{\prime}(v / r)=0, \\
& \text { subject to : } \quad \begin{cases}v(r, 0)=r \phi(r), & 0 \leq r<L, \\
\frac{\partial v}{\partial t}(r, 0)=r \psi(r), & 0 \leq r<L, \\
v(0, t)=0, & t \geq 0\end{cases}
\end{aligned}
$$

Suppose that $a<L$ and $T$ are positive, and that $a$ is approximately equal to $L$. To discretize the differential equation in (3.3) we approximate solutions for values of $r$ and $t$ in the intervals $[0, a]$ and $[0, T]$, respectively. We construct partitions $0=r_{0}<r_{1}<\cdots<r_{M}=a$ and $0=t_{0}<t_{1}<\cdots<t_{N}=T$ of $[0, a]$ and $[0, T]$ consisting of $M$ and $N$ regular subintervals of lengths $\Delta r=a / M$ and $\Delta t=T / N$, respectively.

Denote the approximate value of $v\left(r_{j}, t_{n}\right)$ by $v_{j}^{n}$, for $j=0,1, \ldots, M$ and $n=0,1, \ldots, N$. For the first time step the first initial condition gives $v_{j}^{0}=r_{j} \phi\left(r_{j}\right)$, for each $j=0,1, \ldots, M$. Using second-order approximation the second initial condition yields

$$
v_{j}^{1}=v_{j}^{0}+\left[v_{j-1}^{0}-2 v_{j}^{0}+v_{j+1}^{0}\right]\left(\frac{\Delta t}{\Delta r}\right)^{2}+r_{j} \psi\left(r_{j}\right) \Delta t,
$$

for every $j=1, \ldots, M-1$. The boundary condition in problem (3.3) states that $v_{0}^{n}$ is zero for every $n=0,1, \ldots, N$. Moreover, we impose the constraint that every $v_{m}^{n}$ is also equal to zero in our scheme since we expect solutions to be small at infinity.

Assuming that the solution of (3.3) has been approximated up to the $n$ th time step, we proceed to approximate the solution of the $(n+1)$-st time step by induction, using central second-differences to estimate the second-order partial derivatives of $v$ with respect to $t$ and $r$. The term $v\left(r_{j}, t_{n}\right)$ is approximated using the average of $v_{j}^{n+1}$ and $v_{j}^{n-1}$, and $G^{\prime}\left(v\left(r_{j}, t_{n}\right) / r_{j}\right)$ is estimated using the average rate of change of $G$ between $v_{j}^{n+1} / r_{j}$ and $v_{j}^{n-1} / r_{j}$. Our finite-difference scheme is thus 


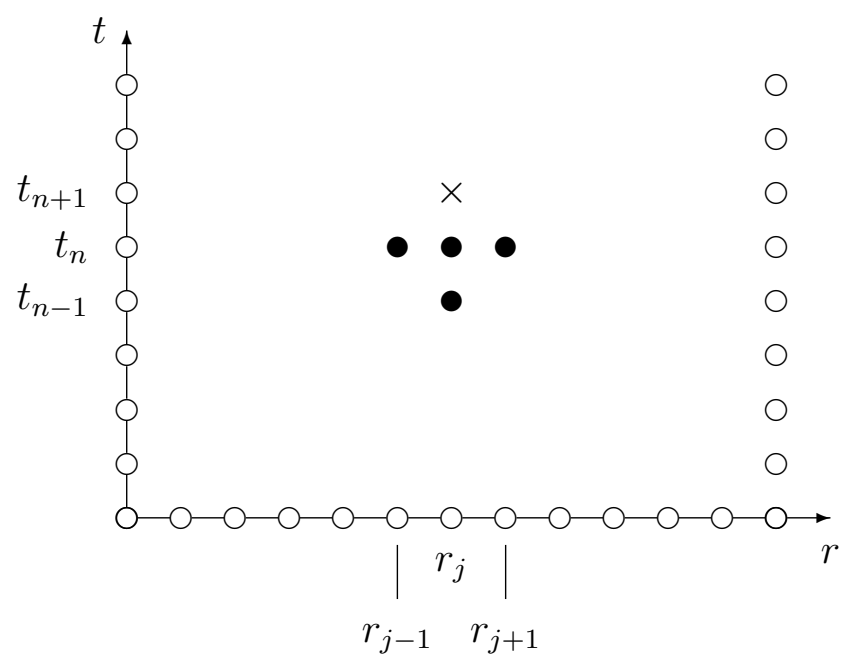

FIGURE 3.1: Forward-difference scheme for the nonlinear Klein-Gordon equation.

$$
\begin{array}{r}
\frac{v_{j}^{n+1}-2 v_{j}^{n}+v_{j}^{n-1}}{(\Delta t)^{2}}-\frac{v_{j+1}^{n}-2 v_{j}^{n}+v_{j-1}^{n}}{(\Delta r)^{2}}+\frac{m^{2}}{2}\left[v_{j}^{n+1}+v_{j}^{n-1}\right]+ \\
r_{j}^{2} \frac{G\left(v_{j}^{n+1} / r_{j}\right)-G\left(v_{j}^{n-1} / r_{j}\right)}{v_{j}^{n+1}-v_{j}^{n-1}}=0 .
\end{array}
$$

The method is explicit if $G$ is constant. If $G$ is not constant the approximation $v_{j}^{n+1}$ can be obtained from Newton's method, keeping all other terms constant. More precisely, let $y_{0}$ be an estimate of the true value of $v_{j}^{n}$. Define

$$
\begin{gathered}
F(y)=\frac{y-2 v_{j}^{n}+v_{j}^{n-1}}{(\Delta t)^{2}}-\frac{v_{j+1}^{n}-2 v_{j}^{n}+v_{j-1}^{n}}{(\Delta r)^{2}}+\frac{m^{2}}{2}\left[y+v_{j}^{n-1}\right] \\
+r_{j}^{2} \frac{G\left(y / r_{j}\right)-G\left(v_{j}^{n-1} / r_{j}\right)}{y-v_{j}^{n-1}} .
\end{gathered}
$$

Then

$$
F^{\prime}(y)=\frac{1}{(\Delta t)^{2}}+\frac{m^{2}}{2}+r_{j}^{2} \frac{G\left(v_{j}^{n-1} / r_{j}\right)-G\left(y / r_{j}\right)+\left(y-v_{j}^{n-1}\right) G^{\prime}\left(y / r_{j}\right)}{\left(y-v_{j}^{n-1}\right)^{2}} .
$$

For initial approximations sufficiently close, Newton's method guarantees that the true value of $v_{j}^{n+1}$ is the limit at which the recursive sequence $y_{n+1}=y_{n}-$ $F\left(y_{n}\right) / F^{\prime}\left(y_{n}\right)$ converges. The order of convergence is quadratic in such circumstances.

As mentioned earlier in Chapter 2, it is physically interesting to approximate radially symmetric solutions of the nonlinear Klein-Gordon equation when $G^{\prime}(u)=u^{p}$, for $p>1$ an odd number. For this choice of $G^{\prime}$, our finite-difference scheme has the simpler expression 


$$
\begin{array}{r}
\frac{v_{j}^{n+1}-2 v_{j}^{n}+v_{j}^{n-1}}{(\Delta t)^{2}}-\frac{v_{j+1}^{n}-2 v_{j}^{n}+v_{j-1}^{n}}{(\Delta r)^{2}}+\frac{m^{2}}{2}\left[v_{j}^{n+1}+v_{j}^{n-1}\right]+ \\
\frac{1}{(j \Delta r)^{p-1}} \frac{G\left(v_{j}^{n+1}\right)-G\left(v_{j}^{n-1}\right)}{v_{j}^{n+1}-v_{j}^{n-1}}=0 .
\end{array}
$$

We state the most important numerical properties of our scheme in the following theorem, whose proof will be given in a more general context in Chapter 4 . For a proof of consistency and stability in case that $G^{\prime}$ and $m$ are both equal to zero, refer to [32].

Theorem 1. Let $G^{\prime}$ be identically equal to zero. Then scheme (3.4) is consistent $\mathcal{O}\left(\Delta t^{2}\right)+\mathcal{O}\left(\Delta r^{2}\right)$. Moreover, it is stable (and thus convergent) if

$$
\left(\frac{\Delta t}{\Delta r}\right)^{2}<1+\frac{1}{4}(m \Delta t)^{2}
$$

\subsection{Energy analysis}

The present section is primarily devoted to compute an expression for the total energy of the three-dimensional nonlinear Klein-Gordon equation. We prove that the energy is conserved throughout time and develop a numeric scheme for the discrete energy that has the same conservative property.

\subsubsection{Derivation of the energy equation}

In order to derive the energy expression of the three-dimensional nonlinear Klein-Gordon equation, it is necessary to compute the Lagrangian and the Hamiltonian of the differential equation in (3.1). Throughout we will use equations from the Lagrangian and Hamiltonian formulations of mechanics. We refer the reader to [33] for more details.

Lemma 2. The Lagrangian of the nonlinear Klein-Gordon equation is given by

$$
\mathcal{L}=\frac{1}{2}\left\{\left(\frac{\partial u}{\partial t}\right)^{2}-|\nabla u|^{2}-m^{2} u^{2}\right\}-G(u)
$$

Proof. Let $x_{0}=t, x_{1}=x, x_{2}=y$, and $x_{3}=z$. To simplify notation, we define

$$
\dot{u}_{i}=\frac{\partial u}{\partial x_{i}}, \quad \text { for } i=0,1,2,3 \text {. }
$$


To prove our lemma, we must verify that the Euler-Lagrange equation associated with $\mathcal{L}$ yields the nonlinear Klein-Gordon equation. The canonical EulerLagrange equation in this case reads

$$
\sum_{i=0}^{4} \frac{\partial}{\partial x_{i}}\left(\frac{\partial \mathcal{L}}{\partial \dot{u}_{i}}\right)-\frac{\partial \mathcal{L}}{\partial u}=0
$$

Notice that

$$
\begin{aligned}
\frac{\partial \mathcal{L}}{\partial u} & =-m^{2} u-G^{\prime}(u), \quad \text { and } \\
\frac{\partial}{\partial x_{i}}\left(\frac{\partial \mathcal{L}}{\partial \dot{u}_{i}}\right) & = \begin{cases}\frac{\partial^{2} u}{\partial t^{2}}, & \text { if } i=0, \\
-\frac{\partial^{2} u}{\partial x_{i}^{2}}, & \text { if } i=1,2,3 .\end{cases}
\end{aligned}
$$

The result is now evident.

Theorem 3. The Hamiltonian of the nonlinear Klein-Gordon equation is given by

$$
\mathcal{H}=\frac{1}{2}\left\{\left(\frac{\partial u}{\partial t}\right)^{2}+|\nabla u|^{2}+m^{2} u^{2}\right\}+G(u) .
$$

COROLlary 4. The total energy associated with the nonlinear Klein-Gordon equation at time $t$ is given by

$$
E(t)=\iiint_{D}\left\{\frac{1}{2}\left(\frac{\partial u}{\partial t}\right)^{2}+\frac{1}{2}|\nabla u|^{2}+\frac{m^{2}}{2} u^{2}+G(u)\right\} d \bar{x} .
$$

Suppose that the solution $u$ of the nonlinear Klein-Gordon equation has radial symmetry at every time $t$ and that $D$ represents the open sphere with center in the origin and radius $L$. Recall that the general expression of the gradient in spherical coordinates is given by

$$
\nabla u=\frac{\partial u}{\partial r} \hat{\mathbf{r}}+\frac{1}{r} \frac{\partial u}{\partial \theta} \hat{\theta}+\frac{1}{r \sin \theta} \frac{\partial u}{\partial \phi} \hat{\phi}
$$

Using this fact, the total energy can be rewritten as

$$
E(t)=4 \pi \int_{0}^{L}\left\{\frac{1}{2}\left(\frac{\partial u}{\partial t}\right)^{2}+\frac{1}{2}\left(\frac{\partial u}{\partial r}\right)^{2}+\frac{m^{2}}{2} u^{2}+G(u)\right\} r^{2} d r
$$

and using the change of variable $v(r, t)=r u(r, t)$, the energy expression for radially symmetric solutions of the nonlinear Klein-Gordon equation adopts the following form. 
COROLlaRY 5. The energy expression at time $t$ of a radially symmetric solution $u$ of the nonlinear Klein-Gordon equation has the form $E(t)=4 \pi E_{0}(t)$, where

$$
E_{0}(t)=\int_{0}^{L}\left\{\frac{1}{2}\left(\frac{\partial v}{\partial t}\right)^{2}+\frac{1}{2}\left(\frac{\partial v}{\partial r}\right)^{2}+\frac{m^{2}}{2} v^{2}+r^{2} G(v / r)\right\} d r
$$

and $u(r, t)=r v(r, t)$.

COROLlary 6. The energy expression at time $t$ of a radially symmetric solution $u$ of the nonlinear Klein-Gordon equation with $G^{\prime}(u)=u^{p}$ has the form $E(t)=4 \pi E_{0}(t)$, where

$$
E_{0}(t)=\int_{0}^{L}\left\{\frac{1}{2}\left(\frac{\partial v}{\partial t}\right)^{2}+\frac{1}{2}\left(\frac{\partial v}{\partial r}\right)^{2}+\frac{m^{2}}{2} v^{2}+r^{1-p} G(v)\right\} d r
$$

and $u(r, t)=r v(r, t)$.

\subsubsection{Continuous energy}

One of the most interesting properties of the energy expression in Corollary 4 is that it is unchanged in time under certain analytical conditions. This statement is proved in Theorem 8 and requires the following result whose proof can be found in [34].

Lemma 7. Let $\phi$ and $\psi$ scalar functions on a domain $D$ of $\mathbb{R}^{3}$ with continuous partial derivatives in $D$. Then for every closed simple domain $R \subseteq D$ with boundary $\partial R$,

$$
\iint_{\partial R} \psi \nabla \phi \cdot \hat{\mathrm{n}} d \sigma=\iiint_{R}\left(\psi \nabla^{2} \phi+\nabla \psi \cdot \nabla \phi\right) d \bar{x}
$$

where $\hat{\mathrm{n}}$ represents the unit vector normal to the surface $\partial R$.

It is worthwhile noticing that if $D$ is a domain of $\mathbb{R}^{3}$ and if $u$ is a function of $\bar{x}$ and $t$ with continuous partial derivatives in $D \times \mathbb{R}$ up to the second order then

$$
\frac{\partial}{\partial t}|\nabla u|^{2}=2 \nabla u \cdot \nabla\left(\frac{\partial u}{\partial t}\right)
$$

By virtue of Lemma 7, for every closed simple domain $R$ contained in $D$ we have that Green's first identity is satisfied:

$$
\iint_{\partial R} \frac{\partial u}{\partial t} \nabla u \cdot \hat{\mathrm{n}} d \sigma=\iiint_{R}\left(\frac{\partial u}{\partial t} \nabla^{2} u+\frac{1}{2} \frac{\partial}{\partial t}|\nabla u|^{2}\right) d \bar{x} .
$$

Equivalently

$$
\frac{1}{2} \iiint_{R} \frac{\partial}{\partial t}|\nabla u|^{2} d \bar{x}=\iint_{\partial R} \frac{\partial u}{\partial t} \nabla u \cdot \hat{\mathrm{n}} d \sigma-\iiint_{R} \frac{\partial u}{\partial t} \nabla^{2} u d \bar{x} .
$$


TheOREM 8. Let $D$ be a domain in $\mathbb{R}^{3}$, and suppose that $\nabla u \cdot \hat{\mathrm{n}}=0$ on the boundary of $D$ at all times. Then the energy of the nonlinear Klein-Gordon equation is conserved.

Proof. Differentiating the expression of the total energy in Corollary 4 and applying successively Equations (3.6) and (3.1), we obtain

$$
\begin{aligned}
\frac{d E}{d t}(t) & =\iiint_{D} \frac{\partial u}{\partial t}\left\{\frac{\partial^{2} u}{\partial t^{2}}+m^{2} u+G^{\prime}(u)\right\} d \bar{x}+\frac{1}{2} \iiint_{D} \frac{\partial}{\partial t}|\nabla u|^{2} d \bar{x} \\
& =\iiint_{D} \frac{\partial u}{\partial t}\left\{\frac{\partial^{2} u}{\partial t^{2}}-\nabla^{2} u+m^{2} u+G^{\prime}(u)\right\} d \bar{x}+\iint_{\partial D} \frac{\partial u}{\partial t} \nabla u \cdot \hat{\mathrm{n}} d \sigma=0 .
\end{aligned}
$$

If $\phi$ and $\psi$ are supposed to be small at infinity then the solution of the nonlinear Klein-Gordon equation will be likewise small at infinity. This implies in particular that $\nabla u=0$ on the boundary of $D$, whence the following result follows.

Corollary 9. Let $D$ be a domain in $\mathbb{R}^{3}$, and suppose that $\phi$ and $\psi$ are smooth functions of compact support in $D$ which are small at infinity. Then the energy of the nonlinear Klein-Gordon equation is conserved.

Our next result implies in particular that radial solutions of the nonlinear Klein-Gordon equation are bounded outside of every open neighborhood of the origin.

THEOREM 10. Let u be a radially symmetric solution of initial-value problem (2.2) in a sphere with center in the origin and radius $L$, with smooth initial conditions which are small at infinity and total energy $E=4 \pi E_{0}$. Then for every $r>0$ and $t$,

$$
|u(r, t)| \leq \frac{\left(2 E_{0}\right)^{1 / 2}}{r} .
$$

Proof. First observe that for every differentiable function $v(r, t)$,

$$
0 \leq\left(\frac{\partial v}{\partial r}-v\right)^{2}=\left(\frac{\partial v}{\partial r}\right)^{2}-\frac{\partial\left(v^{2}\right)}{\partial r}+v^{2}
$$

Let $u$ be a radially symmetric solution of initial-value problem (2.2), and set $v(r, t)=r u(r, t)$. The expression of the total energy of the nonlinear Klein-Gordon equation in Corollary 5 and the fact that $v(0, t)=0$ for every $t$ imply that

$$
\begin{aligned}
v^{2}(r, t) & =\int_{0}^{r} \frac{\partial\left(v^{2}\right)}{\partial r} d r \\
& \leq \int_{0}^{r}\left\{\left(\frac{\partial v}{\partial r}\right)^{2}+v^{2}\right\} d r \\
& \leq 2 E_{0}
\end{aligned}
$$

for every $r$ and $t$, whence the result follows. 


\subsubsection{Discrete energy}

It becomes now necessary to posses a discrete expression to compute the total energy of the system. In view of the fact that the energy of the nonlinear Klein-Gordon equation is conserved throughout time, it is highly desirable to posses a discrete expression to approximate the total energy $E_{n}$ of the system in the $n$-th time step, such that $E_{n}=E_{n-1}$ for each $n \in \mathbb{N}$.

For the sake of precision, let $0=r_{0}<r_{1}<\cdots<r_{M}=a$ and $0=t_{0}<t_{1}<$ $\cdots<t_{N}=T$ be regular partitions of $[0, a]$ and $[0, T]$ into $M$ and $N$ subintervals of lengths $\Delta r=a / N$ and $\Delta t=T / M$, respectively. Let $v_{j}^{n}$ be the value of the function $v$-defined in Corollary 5 - at $\left(r_{j}, t_{n}\right)$. Strauss and Vázquez [8] propose the scheme

$$
\begin{aligned}
\frac{E_{n}^{0}}{\Delta r}= & \frac{1}{2} \sum_{j=0}^{M-1}\left(\frac{v_{j}^{n+1}-v_{j}^{n}}{\Delta t}\right)^{2}+\frac{1}{2} \sum_{j=0}^{M-1}\left(\frac{v_{j+1}^{n+1}-v_{j}^{n+1}}{\Delta r}\right)\left(\frac{v_{j+1}^{n}-v_{j}^{n}}{\Delta r}\right) \\
& +\frac{m^{2}}{2} \sum_{j=0}^{M-1} \frac{\left(v_{j}^{n+1}\right)^{2}+\left(v_{j}^{n}\right)^{2}}{2}+\sum_{j=1}^{M-1} \frac{G\left(v_{j}^{n+1}\right)+G\left(v_{j}^{n}\right)}{2(j \Delta r)^{p-1}}
\end{aligned}
$$

to approximate the true value of $E_{0}$ in Corollary 5. We claim that this scheme has the property that energy is conserved throughout time. To prove this statement, let $n$ and $j$ be positive integers such that $j<M$ and $n<N$. Notice first of all that

$$
\begin{aligned}
\left(v_{j+1}^{n}-2 v_{j}^{n}+v_{j-1}^{n}\right)\left(v_{j}^{n+1}-v_{j}^{n-1}\right)= & -\left(v_{j+1}^{n+1}-v_{j}^{n+1}\right)\left(v_{j+1}^{n}-v_{j}^{n}\right) \\
& +\left(v_{j+1}^{n}-v_{j}^{n}\right)\left(v_{j+1}^{n-1}-v_{j}^{n-1}\right) \\
& +\left(v_{j+1}^{n+1}-v_{j+1}^{n-1}\right)\left(v_{j+1}^{n}-v_{j}^{n}\right) \\
& -\left(v_{j}^{n+1}-v_{j}^{n-1}\right)\left(v_{j}^{n}-v_{j-1}^{n}\right) .
\end{aligned}
$$

Taking the sum over $j$ from 1 to $M-1$ we notice that the last two terms in the right-hand side of Equation (3.7) form a telescoping series. Using the fact that for every nonnegative integer $n$ the quantities $v_{m}^{n}$ and $v_{0}^{n}$ are both equal to zero, this telescoping series becomes

$$
\sum_{j=1}^{M-1}\left[\left(v_{j+1}^{n+1}-v_{j+1}^{n-1}\right)\left(v_{j+1}^{n}-v_{j}^{n}\right)-\left(v_{j}^{n+1}-v_{j}^{n-1}\right)\left(v_{j}^{n}-v_{j-1}^{n}\right)\right]=-v_{1}^{n}\left(v_{1}^{n+1}-v_{1}^{n-1}\right) .
$$

Multiplying finite-difference equation (3.4) by $\left(v_{j}^{n+1}-v_{j}^{n-1}\right)$ and simplifying,

$$
\begin{aligned}
\frac{\left(v_{j}^{n+1}\right)^{2}-2 v_{j}^{n} v_{j}^{n+1}+2 v_{j}^{n} v_{j}^{n-1}-\left(v_{j}^{n-1}\right)^{2}}{(\Delta t)^{2}}-\frac{\left(v_{j+1}^{n}-2 v_{j}^{n}+v_{j-1}^{n}\right)\left(v_{j}^{n+1}-v_{j}^{n-1}\right)}{(\Delta r)^{2}} & \\
+\frac{m^{2}}{2}\left[\left(v_{j}^{n+1}\right)^{2}-\left(v_{j}^{n-1}\right)^{2}\right]+\frac{1}{(j \Delta r)^{p-1}}\left[G\left(v_{j}^{n+1}\right)-G\left(v_{j}^{n-1}\right)\right] & =0 .
\end{aligned}
$$

Next we add the terms $\left(v_{j}^{n}\right)^{2}$ to the numerators of the first and second terms, and $G\left(v_{j}^{n}\right)$ to the numerator of the last term in the left-hand side of this last equation. By regrouping and using Equation (3.7) we obtain 


$$
\begin{aligned}
0= & \left\{\left(\frac{v_{j}^{n+1}-v_{j}^{n}}{\Delta t}\right)^{2}+\left(\frac{v_{j+1}^{n+1}-v_{j}^{n+1}}{\Delta r}\right)\left(\frac{v_{j+1}^{n}-v_{j}^{n}}{\Delta r}\right)+\frac{m^{2}}{2}\left[\left(v_{j}^{n+1}\right)^{2}+\left(v_{j}^{n}\right)^{2}\right]\right. \\
& \left.+\frac{G\left(v_{j}^{n+1}\right)+G\left(v_{j}^{n}\right)}{(j \Delta r)^{p-1}}\right\}-\left\{\left(\frac{v_{j}^{n}-v_{j}^{n-1}}{\Delta t}\right)^{2}+\left(\frac{v_{j+1}^{n}-v_{j}^{n}}{\Delta r}\right)\left(\frac{v_{j+1}^{n-1}-v_{j}^{n-1}}{\Delta r}\right)\right. \\
& \left.+\frac{m^{2}}{2}\left[\left(v_{j}^{n}\right)^{2}+\left(v_{j}^{n-1}\right)^{2}\right]+\frac{G\left(v_{j}^{n}\right)+G\left(v_{j}^{n-1}\right)}{(j \Delta r)^{p-1}}\right\} \\
& -\left[\frac{\left(v_{j+1}^{n+1}-v_{j+1}^{n-1}\right)\left(v_{j+1}^{n}-v_{j}^{n}\right)}{(\Delta r)^{2}}-\frac{\left(v_{j}^{n+1}-v_{j}^{n-1}\right)\left(v_{j}^{n}-v_{j-1}^{n}\right)}{(\Delta r)^{2}}\right] .
\end{aligned}
$$

Finally, we take half of the sum over $j$ from 1 to $M-1$ in both sides of this equation. Observe that the sum of the first four terms differs from twice the total discrete energy in the $n$-th time step by $v_{1}^{n} v_{1}^{n+1} /(\Delta r)^{2}$. Similarly, the sum of the next four terms differs from twice the total discrete energy in the $(n-1)$-st time step by $v_{1}^{n-1} v_{1}^{n} /(\Delta r)^{2}$. Thus it follows that

$$
\left[E_{n}^{0}-\frac{v_{1}^{n} v_{1}^{n+1}}{2(\Delta r)^{2}}\right]-\left[E_{n-1}^{0}-\frac{v_{1}^{n-1} v_{1}^{n}}{2(\Delta r)^{2}}\right]+\frac{v_{1}^{n}\left(v_{1}^{n+1}-v_{1}^{n-1}\right)}{2(\Delta r)^{2}}=0 .
$$

We conclude that $E_{n}^{0}=E_{n-1}^{0}$ for every $n \in \mathbb{N}$ and, in particular, $E_{n}^{0}=E_{0}^{0}$.

\subsection{Numerical results}

Throughout this section we approximate radially symmetric solutions of the nonlinear Klein-Gordon equation with $m^{2}=1$ and $G^{\prime}(u)=u^{p}$, for $p>1$ and odd number.

Let $p=7$ and let the space and time steps be $\Delta r=\Delta t=0.002$. As initial data we choose $\phi(r)=h(r)$ and $\psi(r)=h^{\prime}(r)+h(r) / r$, where

$$
h(r)= \begin{cases}5 \exp \left\{100\left[1-\frac{1}{1-(10 r-1)^{2}}\right]\right\}, & \text { if } 0 \leq r<0.2 \\ 0, & \text { if } 0.2 \leq r \leq 0.4\end{cases}
$$

The results at successive time intervals $t=0,0.04,0.08,0.12,0.16,0.2$ - depicted in Figure 3.2-are in agreement with Strauss and Vázquez [8]. The total energy is effectively conserved and equal to 67.85. Graphically, the initial data immediately break up into an incoming part (moving toward the origin) and an outgoing part (moving away from the origin). The incoming part resolves into oscillations. By time $t=0.16$, the incoming part does not produce new oscillations and all the existing ones have already been reflected away from the origin; all the oscillations from this time on move at the same speed (which appears to be equal to one). We notice that 

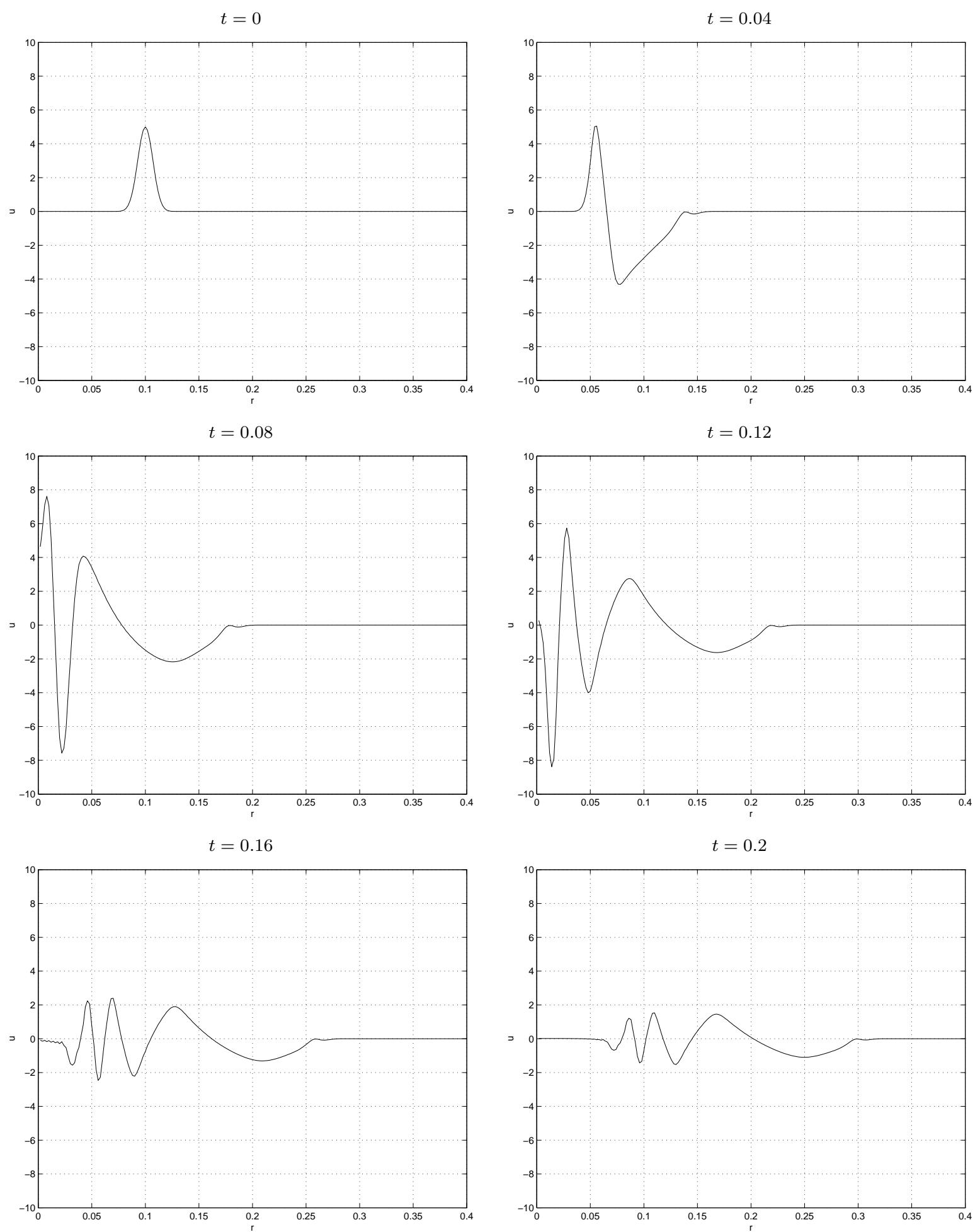

Figure 3.2: Approximate radial solutions at successive times of the nonlinear KleinGordon equation with $G^{\prime}(u)=u^{7}$, initial data $\phi(r)=h(r), \psi(r)=h^{\prime}(r)+h(r) / r$, and boundary condition $u(0.4, t)=0$. 

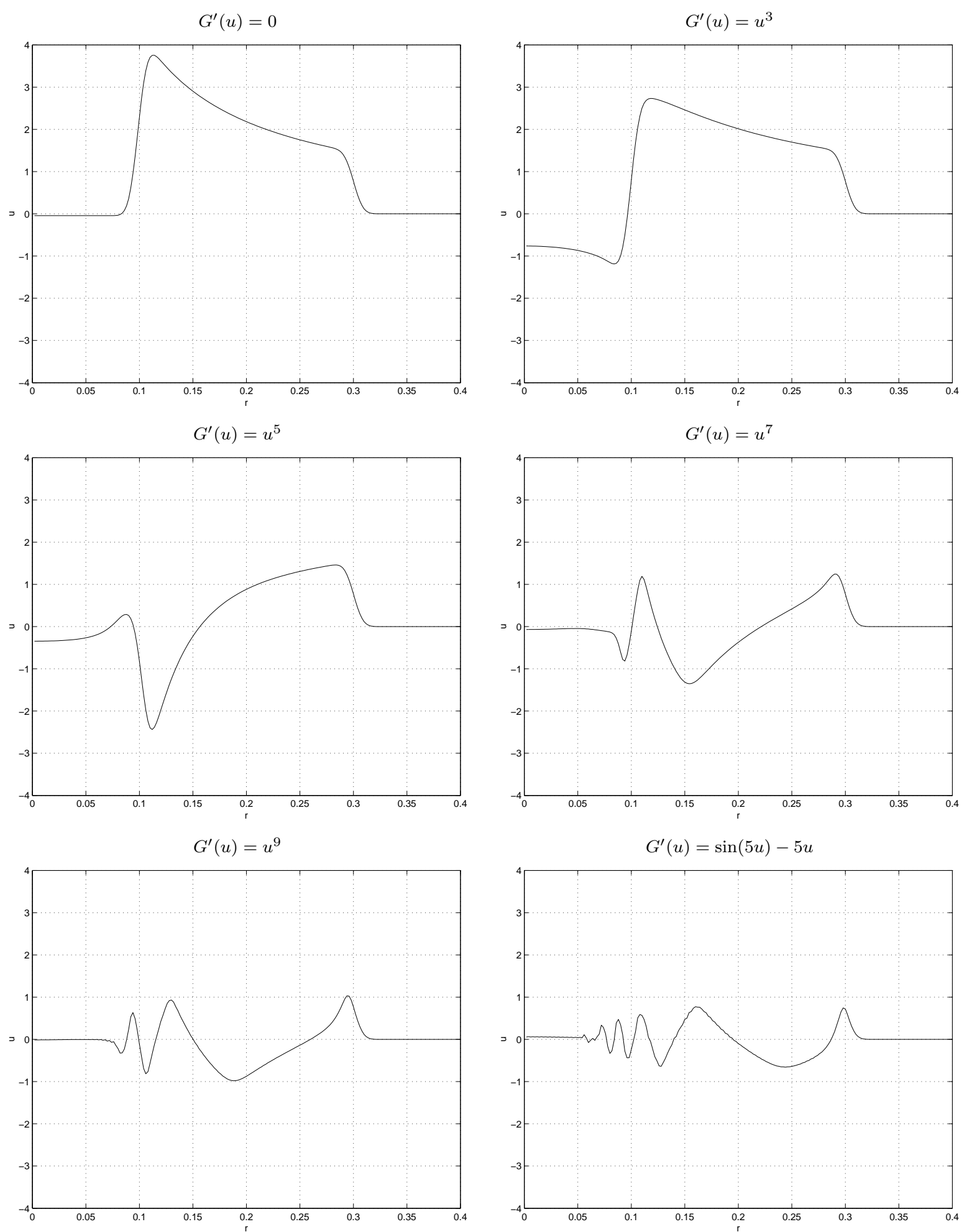

Figure 3.3: Approximate radial solutions for the given function $G^{\prime}(u)$ at time $t=0.2$ for initial data $\phi(r)=0$ and $\psi(r)=100 h(r)$, and boundary condition $u(0.4, t)=0$. 
$G^{\prime}(u)=u^{3}$

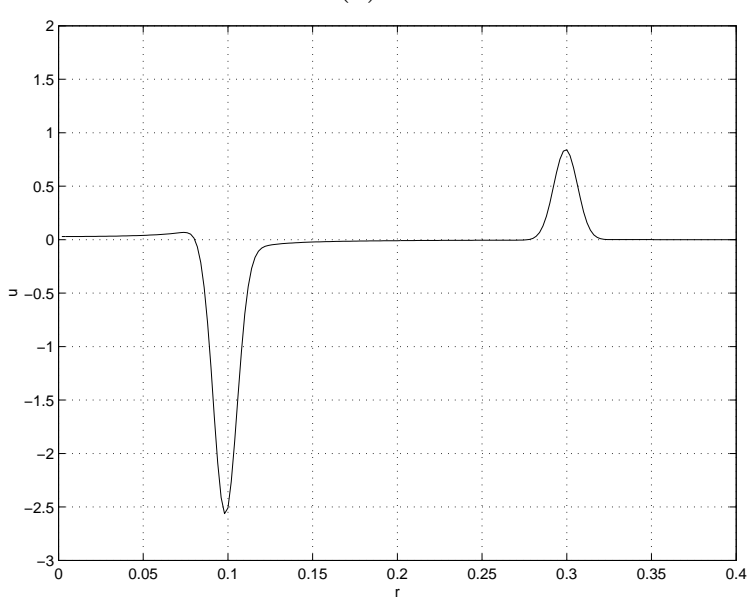

$G^{\prime}(u)=u^{5}$

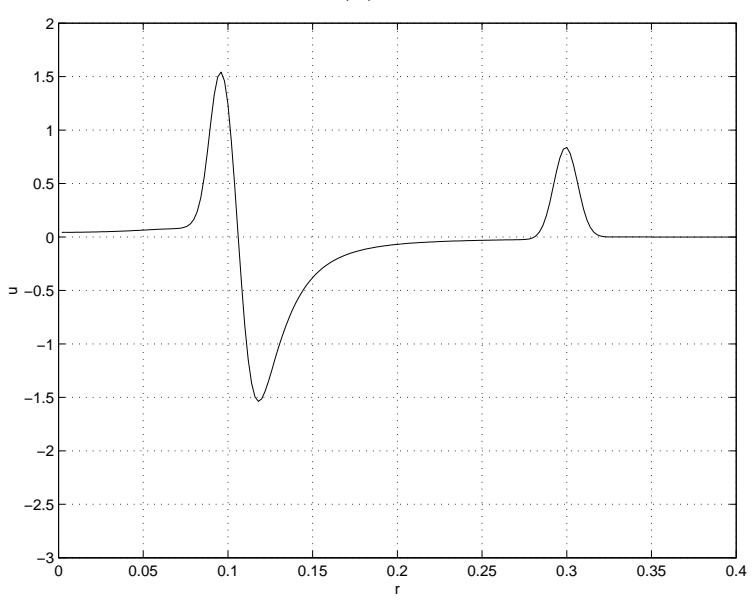

$G^{\prime}(u)=u^{7}$

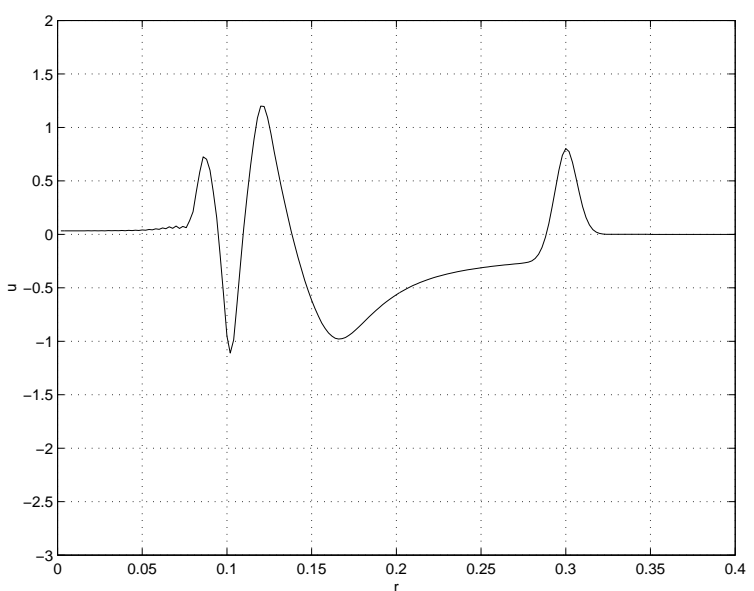

$G^{\prime}(u)=u^{3}$

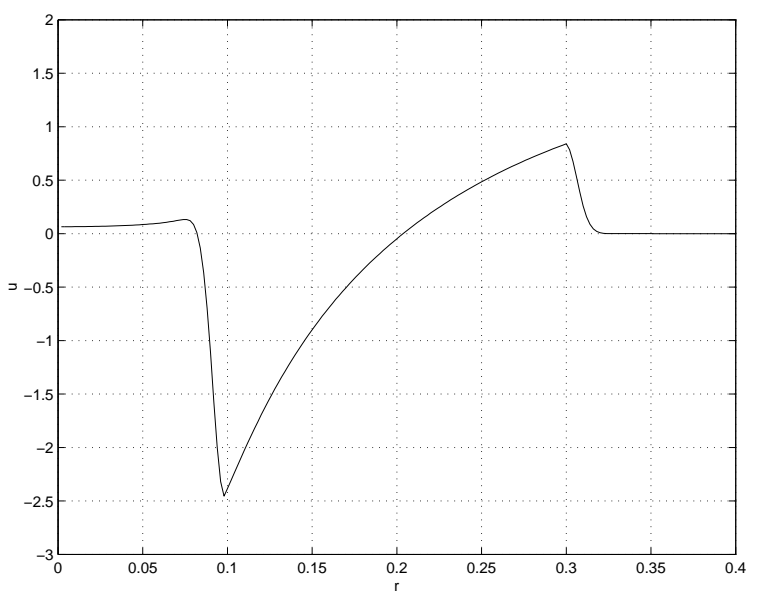

$G^{\prime}(u)=u^{5}$

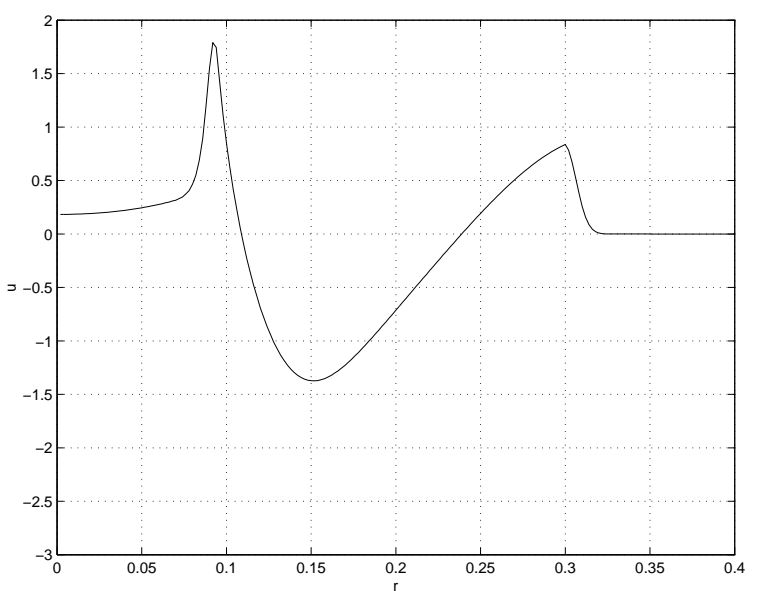

$G^{\prime}(u)=u^{7}$

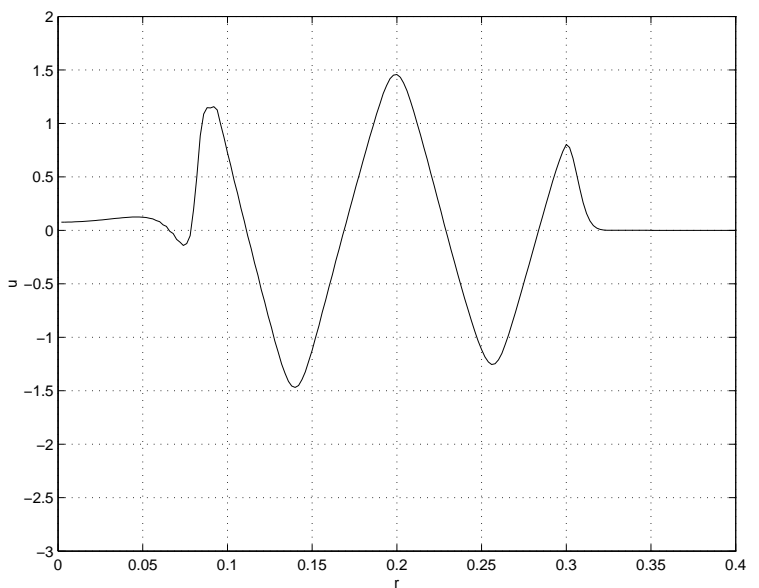

Figure 3.4: Approximate radial solutions for the given function $G^{\prime}(u)$ at time $t=0.2$ for initial data $\phi(r)=h(r), \psi(r)=0$ on the left column, and initial conditions (3.8) on the right column. 
the solutions are bounded at all time, which is in agreement with [6]. Moreover, it appears that $\max _{r \in[0,0.4]}|u(r, t)|$ is a decreasing function of time for values of $t>0.16$.

\begin{tabular}{|c|c|c|c|c|c|c|}
\hline & \multicolumn{6}{|c|}{$G^{\prime}(u)$} \\
\cline { 2 - 7 } & 0 & $u^{3}$ & $u^{5}$ & $u^{7}$ & $u^{9}$ & $\sinh (5 u)-5 u$ \\
\hline $\max _{(r, t)}|u(r, t)|$ & 49.98 & 47.59 & 19.09 & 9.74 & 6.56 & 3.72 \\
\hline
\end{tabular}

TABLE 3.1: Maximum amplitudes over space and time of solutions of the nonlinear KleinGordon equation for six nonlinear terms $G^{\prime}(u)$.

We wish to study now the effect of the nonlinear term. We approximated solutions at time $t=0.2$ for the initial data $\phi(r)=0$ and $\psi(r)=100 h(r)$, and six different equations: $G^{\prime}(u)=0, u^{3}, u^{5}, u^{7}, u^{9}$, and $\sinh (5 u)-5 u$. The results are shown in Figure 3.3 for $\Delta r=\Delta t=0.002$. We observe that the amplitude of the curves seems to decrease as the degree of $G^{\prime}(u)$ increases. Indeed, our computations show that the maximum amplitude decreases with respect to the exponent as Table 3.1 shows. The energy for all powers is equal to 31.294; for the last case the energy equals 31.317 .

Next we computed solutions to the nonlinear Klein-Gordon equation at time $t=0.2$ for the initial data $\phi(r)=h(r)$ and $\psi(r)=0$, and for $p=3,5,7$. The results are printed in the left column of Figure 3.4; the right column shows the solutions of the nonlinear Klein-Gordon equation at the same time and nonlinear term similar to the corresponding graph to the left, for initial data

$$
\phi(r)=\left\{\begin{array}{ll}
5, & \text { for } 0 \leq r \leq 0.1 \\
h(r), & \text { for } 0.1<r \leq 0.4
\end{array} \quad \text { and } \quad \psi(r)=0 .\right.
$$

As before, we verify that the number of oscillations increases with the exponent $p$ of the nonlinear term. Similarly, the amplitude over space and time decreases as the degree of the nonlinear term increases. For each case the energy is conserved; however, the values of the total energy are different for the six cases presented in Figure 3.4.

We finally wish to mention that we have computed solutions of the nonlinear Klein-Gordon equation with nonlinear term given by $G^{\prime}(u)=u^{2}, u^{4}, u^{6}$ and we found out that their graphs blow out in finite time. This is in agreement with [5].

\subsection{Discussion}

Our numerical analysis of the nonlinear Klein-Gordon equation yields the following conclusions:

1. For a fixed nonlinear term and initial data, the total energy is conserved throughout time. 
2. For any initial data the equation immediately breaks up into an incoming part and an outgoing part. The incoming part moves toward the origin while the outgoing moves away from it. The incoming part resolves in oscillations and is reflected away at the origin.

3. From certain time on (about $t=0.16$ in our experiments) the incoming part does not produce new oscillations and all the oscillations move away from the origin at the same speed.

4. The number of final oscillations in the solutions increases with the exponent $p$ of the nonlinear term.

5. The amplitude of the curves over space and time seems to decrease as $p$ increases. Similarly, for a fixed value of $p$ the amplitude of the solution decreases as time increases. 


\title{
Chapter 4
}

\section{A modified nonlinear Klein-Gordon equation}

\begin{abstract}
Dissipative Klein-Gordon-like equations appear in areas of physics as diverse as superconductivity, statistical mechanics and wave mechanics. In view of the fact that there is no general method to compute exact solutions for these equations, it is indispensable to posses reliable numerical techniques to approximate their solutions.

In this chapter we generalize the finite-difference scheme derived in Chapter 3 to account for internal and external damping, and demonstrate consistency and stability of our method. We derive continuous and discrete energy expressions, and compute their rates of change with respect to time. Finally we verify that our method is in general agreement with the undamped nonlinear Klein-Gordon equation, and study the effects of external and internal damping.
\end{abstract}

\section{$4.1 \quad$ Finite-difference scheme}

In this section we prove that the problem of computing radially symmetric solutions of certain dissipative nonlinear Klein-Gordon-like equations is equivalent to a number of problems that have the advantage that are dimensionally simpler or involve solving certain modified nonlinear equation without external damping. For the two simplest formulations we derive discrete schemes based on modifications of the numerical method previously proposed in Chapter 4.

For the sake of simplicity, once and for all we state that the problems we will study in this chapter are of the form (2.2), and convey to call modified KleinGordon equations to any equation resembling the one in the problem under study.

\subsubsection{Equivalent formulations}

Assume that $w$ is a function of position $\bar{x}$ and time $t$, where $\bar{x}$ belongs to a domain $D$ of $\mathbb{R}^{3}$. Suppose that $\phi$ and $\psi$ are smooth functions of $\bar{x}$ of compact support in $D$, and let $G^{\prime}(w)=w^{p}$. The problem we study in this chapter is 


$$
\begin{gathered}
\frac{\partial^{2} w}{\partial t^{2}}-\nabla^{2} w-\beta \frac{\partial}{\partial t}\left(\nabla^{2} w\right)+\gamma \frac{\partial w}{\partial t}+m^{2} w+G^{\prime}(w)=0, \\
\text { subject to : } \begin{cases}w(\bar{x}, 0)=\phi(\bar{x}), & \bar{x} \in D, \\
\frac{\partial w}{\partial t}(\bar{x}, 0)=\psi(\bar{x}), & \bar{x} \in D .\end{cases}
\end{gathered}
$$

Lemma 1. Let $\alpha=1+\beta \gamma / 2$ and $\mu^{2}=m^{2}-\gamma^{2} / 4$. A function $w$ of $(\bar{x}, t)$ is a solution of initial-value problem (4.1) iff $u(\bar{x}, t)=e^{\gamma t / 2} w(\bar{x}, t)$ is a solution of

$$
\begin{aligned}
& \frac{\partial^{2} u}{\partial t^{2}}-\alpha \nabla^{2} u-\beta \frac{\partial}{\partial t}\left(\nabla^{2} u\right)+\mu^{2} u+e^{\gamma t(1-p) / 2} G^{\prime}(u)=0, \\
& \text { subject to : } \quad \begin{cases}u(\bar{x}, 0)=\phi(\bar{x}), & \bar{x} \in D, \\
\frac{\partial u}{\partial t}(\bar{x}, 0)=\frac{\gamma}{2} \phi(\bar{x})+\psi(\bar{x}), & \bar{x} \in D .\end{cases}
\end{aligned}
$$

Proof. Assume first that $w$ satisfies initial-value problem (4.1). Notice that

$$
\begin{aligned}
e^{\gamma t(1-p) / 2} G^{\prime}(u) & =e^{\gamma t / 2} G^{\prime}(w) \\
\nabla^{2} u & =e^{\gamma t / 2} \nabla^{2} w \\
\frac{\partial}{\partial t}\left(\nabla^{2} u\right) & =e^{\gamma t / 2}\left(\frac{\gamma}{2} \nabla^{2} w+\frac{\partial}{\partial t}\left(\nabla^{2} w\right)\right), \quad \text { and } \\
\frac{\partial^{2} u}{\partial t^{2}} & =e^{\gamma t / 2}\left(\frac{\partial^{2} w}{\partial t^{2}}+\gamma \frac{\partial w}{\partial t}+\frac{\gamma^{2}}{4} w\right)
\end{aligned}
$$

It is easy to check that $u$ satisfies the second-order partial differential equation in (4.2). The expression for the first partial derivative of $u$ with respect to $t$ implies that the initial conditions satisfied by $u$ are precisely those described in (4.2).

Conversely, suppose that $u$ satisfies the initial-value problem (4.2). Then

$$
\begin{aligned}
\nabla^{2} w & =e^{-\gamma t / 2} \nabla^{2} u \\
\frac{\partial}{\partial t}\left(\nabla^{2} w\right) & =e^{-\gamma t / 2}\left(-\frac{\gamma}{2} \nabla^{2} u+\frac{\partial}{\partial t}\left(\nabla^{2} u\right)\right) \\
\frac{\partial w}{\partial t} & =e^{-\gamma t / 2}\left(\frac{\partial u}{\partial t}-\frac{\gamma}{2} u\right), \quad \text { and } \\
\frac{\partial^{2} w}{\partial t^{2}} & =e^{-\gamma t / 2}\left(\frac{\partial^{2} u}{\partial t^{2}}-\gamma \frac{\partial u}{\partial t}+\frac{\gamma^{2}}{4} u\right) .
\end{aligned}
$$

It is straight-forward to check that $w$ satisfies the second-order partial differential equation and the initial conditions of (4.1).

COROllary 2. Let $\phi$ and $\psi$ be radially symmetric in an open sphere D, and let $r=\|\bar{x}\|$. Then $w$ is a radially symmetric solution of initial-value problem (4.1) iff $u(r, t)=e^{\gamma t / 2} w(r, t)$ is a radially symmetric solution of $(4.2)$. 
Suppose that $\phi$ and $\psi$ are radially symmetric and that $w(r, t)$ is a radially symmetric solution of initial-value problem (4.2). For every $t>0, w$ satisfies the initial-value problem

$$
\begin{gathered}
\frac{\partial^{2} w}{\partial t^{2}}-\frac{\partial^{2} w}{\partial r^{2}}-\frac{2}{r} \frac{\partial w}{\partial r}-\beta \frac{\partial^{3} w}{\partial t \partial r^{2}}-\frac{2 \beta}{r} \frac{\partial^{2} w}{\partial t \partial r}+\gamma \frac{\partial w}{\partial t}+m^{2} w+G^{\prime}(w)=0, \\
\text { subject to : } \begin{cases}w(r, 0)=\phi(r), & 0<r<L, \\
\frac{\partial w}{\partial t}(r, 0)=\psi(r), & 0<r<L .\end{cases}
\end{gathered}
$$

Consider the change of variable $w(r, t)=e^{-\gamma t / 2} u(r, t)$. Since $w$ is radially symmetric, by virtue of Corollary $2, u$ is also radially symmetric. Moreover, setting $\alpha$ and $\mu^{2}$ as in Lemma 1 we can rewrite initial-value problem (4.2) in spherical coordinates as

$$
\begin{gathered}
\frac{\partial^{2} u}{\partial t^{2}}-\alpha \frac{\partial^{2} u}{\partial r^{2}}-\frac{2 \alpha}{r} \frac{\partial u}{\partial r}-\beta \frac{\partial^{3} u}{\partial t \partial r^{2}}-\frac{2 \beta}{r} \frac{\partial^{2} u}{\partial t \partial r}+\mu^{2} u+e^{\gamma t(1-p) / 2} G^{\prime}(u)=0, \\
\text { subject to : } \begin{cases}u(r, 0)=\phi(r), & 0<r<L, \\
\frac{\partial u}{\partial t}(r, 0)=\frac{\gamma}{2} \phi(r)+\psi(r), & 0<r<L .\end{cases}
\end{gathered}
$$

More precisely, we have the following result.

TheOREM 3. Let $D$ be the open sphere of center at the origin and radius $L$. Let $w(\bar{x}, t)$ be a function with $\bar{x} \in D$ and $t$, and define $u(\bar{x}, t)=e^{\gamma t / 2} w(\bar{x}, t)$. Suppose that $\phi$ and $\psi$ are radially symmetric functions in $D$. The following are equivalent:

(i) $w(\bar{x}, t)$ is a radially symmetric solution of initial-value problem (4.1).

(ii) $u(\bar{x}, t)$ is a radially symmetric solution of initial-value problem (4.2).

(iii) $w(r, t)$ is a solution of initial-value problem (4.3), where $r=\|\bar{x}\|$.

(iv) $u(r, t)$ is a solution of initial-value problem (4.4), where $r=\|\bar{x}\|$.

\subsubsection{The externally damped formulation}

In this section we describe a numerical method to approximate radially symmetric solutions to our modified nonlinear Klein-Gordon equation using the formulation described in (4.3). If no explicit assumption is made on $G^{\prime}(w)$, we will only suppose that it is a continuous function of $w$.

Assume that $w(r, t)$ is a radially symmetric solution of the modified nonlinear Klein-Gordon equation in an open sphere $D$ with center in the origin and radius $L$, with respective internal and external constant damping coefficients $\beta$ and $\gamma$, and initial data $\phi$ and $\psi$ of compact support and small at infinity in $D$. Let $v(r, t)=r w(r, t)$. It is evident that $v(0, t)=0$ for every $t$. Moreover, substituting Equations (3.2) it is easy to check that $v$ must satisfy the mixed-value problem 


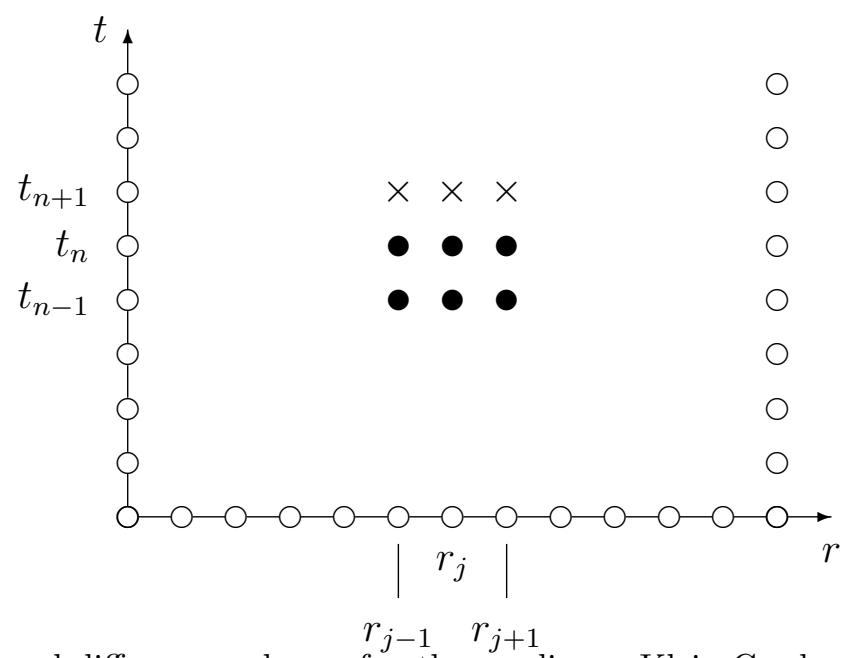

FIGURE 4.1: Forward-difference scheme for the nonlinear Klein-Gordon equation with internal damping.

$$
\begin{aligned}
& \frac{\partial^{2} v}{\partial t^{2}}-\frac{\partial^{2} v}{\partial r^{2}}+\gamma \frac{\partial v}{\partial t}-\beta \frac{\partial^{3} v}{\partial t \partial r^{2}}+m^{2} v+r G^{\prime}(v / r)=0, \quad \text { for } 0<r<L \\
& \text { subject to : } \begin{cases}v(r, 0)=r \phi(r), & 0<r<L, \\
\frac{\partial v}{\partial t}(r, 0)=r \psi(r), & 0<r<L, \\
v(0, t)=0, & t \geq 0\end{cases}
\end{aligned}
$$

Let $a<L$ and $T$ be positive with $a$ close to $L$, and let $0=r_{0}<r_{1}<$ $\cdots<r_{M}=a$ and $0=t_{0}<t_{1}<\cdots<t_{N}=T$ be partitions of $[0, a]$ and $[0, T]$ into $M$ and $N$ subintervals of lengths $\Delta r=a / M$ and $\Delta t=T / N$, respectively. Denote the approximate value of $v\left(r_{j}, t_{n}\right)$ by $v_{j}^{n}$. The first initial condition on $v$ yields $v_{j}^{0}=r_{j} \phi\left(r_{j}\right)$, for every $j=0,1, \ldots, M$, whereas the second gives

$$
v_{j}^{1}=v_{j}^{0}+\left[v_{j-1}^{0}-2 v_{j}^{0}+v_{j+1}^{0}\right]\left(\frac{\Delta t}{\Delta r}\right)^{2}+r_{j} \psi\left(r_{j}\right) \Delta t,
$$

for every $j=1, \ldots, M-1$. The boundary condition guarantees that $v_{0}^{n}$ vanishes for every $n=0,1, \ldots, N$. Moreover, every $v_{M}^{n}$ is also equal to zero in our scheme since we expect solutions to be small at infinity.

Assume that the we have computed the $n$-th approximation to the solution of (4.5). To approximate the solution of the $(n+1)$-st time step we proceed by induction, using central second-differences to estimate the second-order partial derivatives of $v$ with respect to $t$ and $r$, and the difference between $v_{j}^{n+1}$ and $v_{j}^{n-1}$ divided by $2 \Delta t$ to estimate the partial derivative of $v$ with respect to $t$. The third-order derivative corresponding to the internal damping is approximated using central second-differences at times $n-1$ and $n+1$ to estimate the second-order partial derivatives of $v$ with respect to $r$ at $t_{n-1}$ and $t_{n+1}$, and then we compute the difference of those derivatives divided by $2 \Delta t$. The term $v\left(r_{j}, t_{n}\right)$ is approximated using the average of $v_{j}^{n+1}$ and 
$v_{j}^{n-1}$, and $G^{\prime}\left(v\left(r_{j}, t_{n}\right) / r_{j}\right)$ is estimated using the average rate of change of $G$ between $v_{j}^{n+1} / r_{j}$ and $v_{j}^{n-1} / r_{j}$. In these circumstances our finite-difference scheme becomes

$$
\begin{aligned}
& \frac{v_{j}^{n+1}-2 v_{j}^{n}+v_{j}^{n-1}}{(\Delta t)^{2}}-\frac{v_{j+1}^{n}-2 v_{j}^{n}+v_{j-1}^{n}}{(\Delta r)^{2}}+\gamma \frac{v_{j}^{n+1}-v_{j}^{n-1}}{2 \Delta t}- \\
& \beta \frac{\left(v_{j+1}^{n+1}-2 v_{j}^{n+1}+v_{j-1}^{n+1}\right)-\left(v_{j+1}^{n-1}-2 v_{j}^{n-1}+v_{j-1}^{n-1}\right)}{2 \Delta t(\Delta r)^{2}}+ \\
& \frac{m^{2}}{2}\left[v_{j}^{n+1}+v_{j}^{n-1}\right]+r_{j}^{2} \frac{G\left(v_{j}^{n+1} / r_{j}\right)-G\left(v_{j}^{n-1} / r_{j}\right)}{v_{j}^{n+1}-v_{j}^{n-1}}=0 .
\end{aligned}
$$

Several remarks have to be made about this numerical scheme depending on the values assigned to the damping coefficients $\beta$ and $\gamma$, and the expression of the function $G^{\prime}$ :

- If $\beta$ and $\gamma$ are equal to zero and $G^{\prime}$ is identically zero, our equation is the classical Klein-Gordon equation without damping. In this case scheme (4.6) is explicit, consistent, and conditionally stable order $n$.

- If $\beta$ is zero and $G^{\prime}$ is identically zero but $\gamma$ is not equal to zero we obtain the linear Klein-Gordon equation with constant external damping. The scheme associated with this equation is again explicit, and we will prove that it is likewise consistent and conditionally stable order $n$.

- If $\beta$ is zero but neither $\gamma$ nor $G^{\prime}$ is equal to zero then our equation becomes the nonlinear Klein-Gordon equation with constant external damping. The finite-difference scheme associated is nonlinear and implicit. In fact, $v_{j}^{n+1}$ must be obtained using Newton's method in our scheme with $v_{j}^{n+1}$ being variable and all other terms constants ( $c f$. Section 3.1).

- If $G^{\prime}$ is identically zero but neither $\beta$ nor $\gamma$ are equal to zero then our third-order differential equation is linear and the associated finite-difference scheme is likewise linear and implicit. Our scheme can be written as

$$
a v_{j-1}^{n+1}+b v_{j}^{n+1}+a v_{j+1}^{n+1}=\left(c v_{j-1}^{n}+d v_{j}^{n}+c v_{j+1}^{n}\right)+\left(a v_{j-1}^{n+1}+e v_{j}^{n+1}+a v_{j+1}^{n+1}\right)
$$

for every $j=1, \ldots, M-1$ and $n=1, \ldots, N-1$. Here 


$$
\begin{aligned}
a & =\frac{\beta}{2 \Delta t(\Delta r)^{2}}, \\
b & =\frac{\gamma}{2 \Delta t}+\frac{1}{(\Delta t)^{2}}+\frac{m^{2}}{2}+\frac{\beta}{\Delta t(\Delta r)^{2}}, \\
c & =\frac{1}{(\Delta r)^{2}}, \\
d & =2\left(\frac{1}{(\Delta t)^{2}}-\frac{1}{(\Delta r)^{2}}\right), \quad \text { and } \\
e & =\frac{\gamma}{2 \Delta t}-\frac{1}{(\Delta t)^{2}}-\frac{m^{2}}{2}-\frac{\beta}{\Delta t(\Delta r)^{2}} .
\end{aligned}
$$

Let $\bar{v}_{n}^{t}=\left(v_{1}^{n}, v_{2}^{n}, \ldots, v_{M-1}^{n}\right)$, for every $n \in \mathbb{N}$. Recall that $v_{0}^{n}$ and $v_{M}^{n}$ are equal to zero for every $n$, and assume that $\bar{v}_{1}, \bar{v}_{2}, \ldots, \bar{v}_{n}$ have all been computed. Then $\bar{v}_{n+1}$ can be computed following the expression of our finite-difference scheme in matricial form

$$
A \bar{v}_{n+1}=C \bar{v}_{n}+E \bar{v}_{n-1},
$$

where $A, C$, and $E$ are the band matrices

$$
\begin{aligned}
& A=\left(\begin{array}{ccccc}
b & a & 0 & \cdots & 0 \\
a & b & a & \cdots & 0 \\
0 & a & b & \cdots & 0 \\
\vdots & \vdots & \vdots & \ddots & \vdots \\
0 & 0 & 0 & \cdots & b
\end{array}\right), \quad C=\left(\begin{array}{ccccc}
d & c & 0 & \cdots & 0 \\
c & d & c & \cdots & 0 \\
0 & c & d & \cdots & 0 \\
\vdots & \vdots & \vdots & \ddots & \vdots \\
0 & 0 & 0 & \cdots & d
\end{array}\right), \quad \text { and } \\
& E=\left(\begin{array}{ccccc}
e & a & 0 & \cdots & 0 \\
a & e & a & \cdots & 0 \\
0 & a & e & \cdots & 0 \\
\vdots & \vdots & \vdots & \ddots & \vdots \\
0 & 0 & 0 & \cdots & e
\end{array}\right)
\end{aligned}
$$

The matrix equation described above can be solved for $\bar{v}_{n+1}$ by computing $\bar{v}_{n+1}=A^{-1} C \bar{v}_{n}+A^{-1} E \bar{v}_{n-1}$, at the risk of loosing significant decimal places in the approximation to $\bar{v}_{n+1}$ when calculating $A^{-1}$. Alternatively, it is possible to decompose matrix $A$ as the product of a lower-band and an upper-band matrices, and solve the equivalent linear system using Crout reduction for tridiagonal linear systems [35].

- If neither $\beta$ nor $\gamma$ nor $G^{\prime}$ are equal to zero our third-order differential equation is nonlinear and its finite-difference scheme is likewise nonlinear and implicit. The way to approach the solution to this problem is by making use of Newton's method for solving numerically nonlinear systems of equations 
(see [35]) assuming $\bar{v}_{n}$ and $\bar{v}_{n-1}$ constant. The vectors $\bar{v}_{n}$ are defined as in the previous item.

To be more precise, suppose that $F$ is the vector function $\left(f_{1}, f_{2}, \ldots, f_{M-1}\right)$ in the vector variable $\bar{x}=\left(x_{1}, \ldots, x_{M-1}\right)$. Let $x_{0}$ and $x_{M}$ be both equal to zero and, for every $j=1, \ldots, M-1$, let the function $f_{j}$ be given by

$$
\begin{aligned}
f_{j}(\bar{x})=\frac{x_{j}-}{2 v_{j}^{n}+v_{j}^{n-1}}-\frac{v_{j+1}^{n}-2 v_{j}^{n}+v_{j-1}^{n}}{(\Delta t)^{2}}+\gamma \frac{x_{j}-v_{j}^{n-1}}{2 \Delta t} \\
-\beta \frac{\left(x_{j+1}-2 x_{j}+x_{j-1}\right)-\left(v_{j+1}^{n-1}-2 v_{j}^{n-1}+v_{j-1}^{n-1}\right)}{2 \Delta t(\Delta r)^{2}} \\
+\frac{m^{2}}{2}\left[x_{j}+v_{j}^{n-1}\right]+r_{j}^{2} \frac{G\left(x_{j} / r_{j}\right)-G\left(v_{j}^{n-1} / r_{j}\right)}{x_{j}-v_{j}^{n-1}} .
\end{aligned}
$$

The Jacobian matrix $J(\bar{x})$ containing all first-order partial derivatives of the vector function $F(\bar{x})$ is a band matrix. To see this let $j=1, \ldots, M-1$ and $i=1, \ldots, M-1$. Notice that if the absolute value of the difference $i-j$ is greater than 1 then the partial of $f_{j}$ with respect to $x_{i}$ is equal to zero. Otherwise, following the notation of the previous item it is easy to check that

$$
\frac{\partial f_{j}}{\partial x_{i}}= \begin{cases}-a, & i=j-1 \\ b+r_{j} \frac{\left(x_{j}-v_{j}^{n-1}\right) G^{\prime}\left(\frac{x_{j}}{r_{j}}\right)-r_{j}\left[G\left(\frac{x_{j}}{r_{j}}\right)-G\left(\frac{v_{j}^{n-1}}{r_{j}}\right)\right]}{\left(x_{j}-v_{j}^{n-1}\right)^{2}}, & i=j \\ -a, & i=j+1 .\end{cases}
$$

The vector $\bar{v}_{n+1}$ is defined as having the property that $F\left(\bar{v}_{n+1}\right)=0$. Alternatively, it is the limit at which the recursive sequence $\bar{x}_{n+1}=\bar{x}_{n}-$ $J^{-1}\left(\bar{x}_{n}\right) F\left(x_{n}\right)$ converges for a sufficiently close approximation $\bar{x}_{0}$ of $\bar{v}_{n+1}$, in which case the convergence is quadratic [35]. In each iteration the vector $\bar{y}_{n}=J^{-1}\left(\bar{x}_{n}\right) F\left(x_{n}\right)$ will be computed alternatively solving the linear system $J\left(\bar{x}_{n}\right) \bar{y}_{n}=F\left(x_{n}\right)$, using Crout's reduction method for tridiagonal systems.

If $G^{\prime}(w)=w^{p}$ then our modified initial-value problem assumes the form

$$
\begin{aligned}
& \frac{\partial^{2} v}{\partial t^{2}}-\frac{\partial^{2} v}{\partial r^{2}}+\gamma \frac{\partial v}{\partial t}-\beta \frac{\partial^{3} v}{\partial t \partial r^{2}}+m^{2} v+r^{1-p} G^{\prime}(v)=0, \quad \text { for } 0<r<L \\
& \text { subject to : } \begin{cases}v(r, 0)=r \phi(r), & 0<r<L \\
\frac{\partial v}{\partial t}(r, 0)=r \psi(r), & 0<r<L \\
v(0, t)=0, & t \geq 0\end{cases}
\end{aligned}
$$

and our finite-difference scheme has the simpler expression 


$$
\begin{gathered}
\frac{v_{j}^{n+1}-2 v_{j}^{n}+v_{j}^{n-1}}{(\Delta t)^{2}}-\frac{v_{j+1}^{n}-2 v_{j}^{n}+v_{j-1}^{n}}{(\Delta r)^{2}}+\gamma \frac{v_{j}^{n+1}-v_{j}^{n-1}}{2 \Delta t}- \\
\beta \frac{\left(v_{j+1}^{n+1}-2 v_{j}^{n+1}+v_{j-1}^{n+1}\right)-\left(v_{j+1}^{n-1}-2 v_{j}^{n-1}+v_{j-1}^{n-1}\right)}{2 \Delta t(\Delta r)^{2}}+ \\
\frac{m^{2}}{2}\left[v_{j}^{n+1}+v_{j}^{n-1}\right]+\frac{1}{(j \Delta r)^{p-1}} \frac{G\left(v_{j}^{n+1}\right)-G\left(v_{j}^{n-1}\right)}{v_{j}^{n+1}-v_{j}^{n-1}}=0 .
\end{gathered}
$$

\subsubsection{The externally undamped formulation}

As we will prove later, the damped formulation described in the previous section provides us with a conditionally stable scheme that converges to the radial solution of the modified nonlinear Klein-Gordon equation, with order of convergence $\mathcal{O}\left(\Delta r^{2}\right)+\mathcal{O}\left(\Delta t^{2}\right)$. From a practical point of view it is unnecessary to derive a finitedifference scheme for the undamped formulation (4.4). From a theoretic point of view, though, it is an interesting exercise to derive the properties of scheme(4.4).

Let $w(\bar{x}, t)=e^{\gamma t / 2} u(\bar{x}, t)$ and assume that $w$ is a radially symmetric solution to the modified nonlinear Klein-Gordon equation in the open sphere $D$ with center in the origin and radius $L$, with nonlinear term $G^{\prime}(w)=w^{p}$, and radially symmetric data $\phi$ and $\psi$ smooth and of compact support in $D$, and small at infinity. Then $u$ must satisfy (4.4). Moreover, letting $\alpha$ and $\mu^{2}$ be as in Lemma 1 and using the change of variable $v(r, t)=r u(r, t)$, we obtain the mixed-value problem

$$
\begin{aligned}
& \frac{\partial^{2} v}{\partial t^{2}}-\alpha \frac{\partial^{2} v}{\partial r^{2}}+\beta \frac{\partial^{3} v}{\partial t \partial r^{2}}+\mu^{2} v+\left(r e^{\gamma t / 2}\right)^{1-p} G^{\prime}(v)=0, \quad \text { for } 0<r<L, \\
& \text { subject to : } \quad \begin{cases}v(r, 0)=r \phi(r), & 0<r<L, \\
\frac{\partial v}{\partial t}(r, 0)=\left(\frac{\gamma}{2} \phi(r)+\psi(r)\right) r, & 0<r<L, \\
v(0, t)=0, & t \geq 0 .\end{cases}
\end{aligned}
$$

Using the conventions and nomenclature of the preceding section, we must have $v_{j}^{0}=r_{j} \phi\left(r_{j}\right)$ and

$$
v_{j}^{1}=v_{j}^{0}+\left[v_{j-1}^{0}-2 v_{j}^{0}+v_{j+1}^{0}\right]\left(\frac{\Delta t}{\Delta r}\right)^{2}+r_{j}\left(\frac{\gamma}{2} \phi\left(r_{j}\right)+\psi\left(r_{j}\right)\right) \Delta t,
$$

for every $j=1, \ldots, M-1$. Moreover, $v_{0}^{n}$ and $v_{M}^{n}$ are equal to zero for every $n=0,1, \ldots, N$. A discrete scheme associated with (4.9) is described by the finitedifference equation 


$$
\begin{gathered}
\frac{v_{j}^{n+1}-2 v_{j}^{n}+v_{j}^{n-1}}{(\Delta t)^{2}}-\frac{v_{j+1}^{n}-2 v_{j}^{n}+v_{j-1}^{n}}{(\Delta r)^{2}}+\frac{1}{2}\left[v_{j}^{n+1}+v_{j}^{n-1}\right]- \\
\beta \frac{\left(v_{j+1}^{n+1}-2 v_{j}^{n+1}+v_{j-1}^{n+1}\right)-\left(v_{j+1}^{n-1}-2 v_{j}^{n-1}+v_{j-1}^{n-1}\right)}{2 \Delta t(\Delta r)^{2}}+ \\
\frac{1}{\left(e^{\gamma t_{n} / 2} r_{j}\right)^{p-1}} \frac{G\left(v_{j}^{n+1}\right)-G\left(v_{j}^{n-1}\right)}{v_{j}^{n+1}-v_{j}^{n-1}}=0 .
\end{gathered}
$$

\subsubsection{Numerical properties}

In this section we study the numerical properties of consistency and stability of finite-difference scheme (4.8) with $G^{\prime}$ identically equal to zero. We prove that our scheme is consistent $\mathcal{O}\left(\Delta t^{2}\right)+\mathcal{O}\left(\Delta r^{2}\right)$, and derive a necessary stability condition. Throughout we assume that $v(r, t)=r u(r, t)$ has continuous partial derivatives of all orders.

Let $L$ be positive and suppose that $v$ is a solution of initial-value problem (4.7) that has smooth partial derivatives of all orders for every $0<r<L$ and $t$. We use Maclaurin series expansions on $v$ : For every positive increments $\Delta r$ and $\Delta t$, and for every $r$ and $t$ with $\Delta r<r<L-\Delta r$ and $\Delta t<t$,

$$
\begin{array}{ll}
v(r+\Delta r, t) & =\sum_{k=0}^{\infty} \frac{(\Delta r)^{k}}{k !} \frac{\partial^{k} v}{\partial r^{k}}(r, t) \quad \text { and } \\
v(r, t+\Delta t) & =\sum_{k=0}^{\infty} \frac{(\Delta t)^{k}}{k !} \frac{\partial^{k} v}{\partial t^{k}}(r, t) .
\end{array}
$$

As before, we discretize our problem by taking partitions $0=r_{0}<r_{1}<$ $\cdots<r_{M}=a$ and $0=t_{0}<t_{1}<\cdots<t_{N}=T$ of $[0, a]$ and $[0, T]$, consisting of $M$ and $N$ subintervals of lengths $\Delta r=a / M$ and $\Delta t=T / N$, respectively. Denote the approximate value of $v\left(r_{j}, t_{n}\right)$ by $v_{j}^{n}$.

With this conventions observe that

$$
\begin{aligned}
\frac{v_{j}^{n+1}-v_{j}^{n-1}}{2 \Delta t} & =\frac{\partial v}{\partial t}\left(r_{j}, t_{n}\right)+(\Delta t)^{2} \sum_{k=1}^{\infty} \frac{(\Delta t)^{2 k-2}}{(2 k+1) !} \frac{\partial^{2 k+1} v}{\partial t^{2 k+1}}\left(r_{j}, t_{n}\right), \\
\frac{v_{j}^{n+1}-2 v_{j}^{n}+v_{j}^{n-1}}{(\Delta t)^{2}} & =\frac{\partial^{2} v}{\partial t^{2}}\left(r_{j}, t_{n}\right)+(\Delta t)^{2} \sum_{k=2}^{\infty} \frac{2(\Delta t)^{2 k-4}}{(2 k) !} \frac{\partial^{2 k} v}{\partial t^{2 k}}\left(r_{j}, t_{n}\right), \\
\frac{v_{j+1}^{n}-2 v_{j}^{n}+v_{j-1}^{n}}{(\Delta r)^{2}} & =\frac{\partial^{2} v}{\partial r^{2}}\left(r_{j}, t_{n}\right)+(\Delta r)^{2} \sum_{k=2}^{\infty} \frac{2(\Delta r)^{2 k-4}}{(2 k) !} \frac{\partial^{2 k} v}{\partial r^{2 k}}\left(r_{j}, t_{n}\right), \quad \text { and } \\
\frac{v_{j}^{n+1}+v_{j}^{n-1}}{2} & =v\left(r_{j}, t_{n}\right)+(\Delta t)^{2} \sum_{k=1}^{\infty} \frac{(\Delta t)^{2 k-2}}{(2 k) !} \frac{\partial^{2 k} v}{\partial t^{2 k}}\left(r_{j}, t_{n}\right) .
\end{aligned}
$$


We proceed to determine the consistency of the fourth term in scheme (4.8) using Taylor series expansions of scalar functions in two independent variables (see for instance [36]). In the next equations we use the notation $\delta_{k i}$ to represent the number 1 if $k=i$, or 0 if $k \neq i$. The partial derivatives are all evaluated at $\left(r_{j}, t_{n}\right)$.

$$
\begin{aligned}
v_{j+1}^{n+1}-v_{j}^{n} & =\sum_{k=1}^{\infty} \sum_{i=0}^{k} \frac{(\Delta r)^{i}(\Delta t)^{k-i}}{i !(k-i) !} \frac{\partial^{k} v}{\partial r^{i} \partial t^{k-i}}, \\
v_{j-1}^{n+1}-v_{j}^{n} & =\sum_{k=1}^{\infty} \sum_{i=0}^{k}(-1)^{i} \frac{(\Delta r)^{i}(\Delta t)^{k-i}}{i !(k-i) !} \frac{\partial^{k} v}{\partial r^{i} \partial t^{k-i}}, \quad \text { and } \\
v_{j}^{n+1}-v_{j}^{n} & =\sum_{k=1}^{\infty} \sum_{i=0}^{k} \frac{(\Delta r)^{i}(\Delta t)^{k-i}}{i !(k-i) !} \delta_{i 0} \frac{\partial^{k} v}{\partial r^{i} \partial t^{k-i}} .
\end{aligned}
$$

We subtract the double of the second equation from the sum of the first and third equations to obtain a central second-difference that approximates the second partial derivative of $v$ with respect to $r^{2}$ evaluated at $\left(r_{j}, t_{n+1}\right)$. Moreover, changing $\Delta t$ for $-\Delta t$ in the equations above we can obtain a central second difference for the second partial derivative of $v$ with respect to $r^{2}$ at $\left(r_{j}, t_{n-1}\right)$. Using this information we obtain

$$
\left(v_{j+1}^{n+1}-2 v_{j}^{n+1}+v_{j-1}^{n+1}\right)-\left(v_{j+1}^{n-1}-2 v_{j}^{n-1}+v_{j-1}^{n-1}\right)=\sum_{k=1}^{\infty} \sum_{i=0}^{k} \lambda_{k i} \frac{(\Delta r)^{i}(\Delta t)^{k-i}}{i !(k-i) !} \frac{\partial^{k} v}{\partial r^{i} \partial t^{k-i}},
$$

where $\lambda_{k i}=\left[1-2 \delta_{i 0}+(-1)^{i}\right]\left[1-(-1)^{k-i}\right]$, for every $k \in \mathbb{N}$ and $i=0,1, \ldots, k$. Instead of proceeding analytically now we prefer to appeal to Table 4.1, which gives some few values of the coefficients $\lambda_{k i}$. We notice that $\lambda_{32}, \lambda_{52}$ and $\lambda_{54}$ are the first nonzero coefficients in the above difference of central second-differences. Dividing this difference by $2 \Delta t(\Delta r)^{2}$ we get

$$
\begin{gathered}
\frac{\left(v_{j+1}^{n+1}-2 v_{j}^{n+1}+v_{j-1}^{n+1}\right)-\left(v_{j+1}^{n-1}-2 v_{j}^{n-1}+v_{j-1}^{n-1}\right)}{2 \Delta t(\Delta r)^{2}}=\frac{\partial^{3} v}{\partial t \partial r^{2}}+\frac{(\Delta t)^{2}}{120} \frac{\partial^{5} v}{\partial r^{2} \partial t^{3}} \\
+\frac{(\Delta r)^{2}}{12} \frac{\partial^{5} v}{\partial^{4} r \partial t}+\sum_{k=7}^{\infty} \sum_{i=0}^{k} \lambda_{k i} \frac{(\Delta r)^{i}(\Delta t)^{k-i}}{i !(k-i) !} \frac{\partial^{k} v}{\partial r^{i} \partial t^{k-i}} .
\end{gathered}
$$

It is clear now that scheme (4.8) is consistent order $\mathcal{O}\left(\Delta t^{2}\right)+\mathcal{O}\left(\Delta r^{2}\right)$ with initial-value problem (4.7) whenever $G^{\prime}$ is identically equal to zero. Furthermore, we have the following result whose proof makes use of the fact that $\|A\| \geq|\lambda|$, for every squared matrix $A$ and every eigenvalue $\lambda$ of $A$.

THEOREM 4. Let $G^{\prime}$ be identically zero. Then scheme (4.8) is consistent order $\mathcal{O}\left(\Delta t^{2}\right)+\mathcal{O}\left(\Delta r^{2}\right)$ with initial-value problem (4.7). Moreover, in order for the scheme to be stable (and thus convergent) it is necessary that

$$
\left(\frac{\Delta t}{\Delta r}\right)^{2}<1+\gamma \frac{\Delta t}{4}+\beta \frac{\Delta t}{(\Delta r)^{2}}+m^{2} \frac{(\Delta t)^{2}}{4}
$$




\begin{tabular}{|c|c|c|c|c|c|c|c|}
\hline \multirow{2}{*}{$i$} & \multicolumn{7}{|c|}{$k$} \\
\cline { 2 - 8 } & 1 & 2 & 3 & 4 & 5 & 6 & 7 \\
\hline \hline 0 & 0 & 0 & 0 & 0 & 0 & 0 & 0 \\
1 & 0 & 0 & 0 & 0 & 0 & 0 & 0 \\
2 & & 0 & 4 & 0 & 4 & 0 & 4 \\
3 & & & 0 & 0 & 0 & 0 & 0 \\
4 & & & & 0 & 4 & 0 & 4 \\
5 & & & & & 0 & 0 & 0 \\
6 & & & & & & 0 & 4 \\
7 & & & & & & & 0 \\
\hline
\end{tabular}

TABLE 4.1: Coefficients $\lambda_{k i}$ in the difference of central second differences approximating the internal damping term of the nonlinear Klein-Gordon equation.

Proof. Following the nomenclature of this section we define $V_{1 j}^{n+1}=v_{j}^{n+1}$ and $V_{2 j}^{n+1}=v_{j}^{n}$ for each $j=0,1, \ldots, M$ and $n=0,1, \ldots, N-1$. Observe that $V_{1 j}^{n}=v_{j}^{n}$ and $V_{2 j}^{n}=v_{j}^{n-1}$ for $j=0,1, \ldots, M$ and $n=1, \ldots, N-1$. Let $R=\Delta t / \Delta r$. Scheme (4.6) can be rewritten as

$$
\begin{aligned}
\frac{v_{j}^{n+1}-2 v_{j}^{n}+v_{j}^{n-1}}{(\Delta t)^{2}}-\frac{\delta^{2} v_{j}^{n}}{(\Delta r)^{2}}+\gamma \frac{v_{j}^{n+1}-v_{j}^{n-1}}{2 \Delta t}- \\
\beta \frac{\delta^{2} v_{j}^{n+1}-\delta^{2} v_{j}^{n-1}}{2 \Delta t(\Delta r)^{2}}+\frac{m^{2}}{2}\left[v_{j}^{n+1}+v_{j}^{n-1}\right]=0 .
\end{aligned}
$$

For every $j=0,1, \ldots, M$ and $n=0,1, \ldots, N$ let $\bar{V}_{j}^{n}$ be the column vector whose components are $V_{1 j}^{n}$ and $V_{2 j}^{n}$. Solving for $v_{j}^{n+1}$ in our modified numeric scheme and noticing that $V_{2 j}^{n+1}=V_{1 j}^{n}$, our problem can be written in matricial form as

$$
\left(\begin{array}{cc}
k & 0 \\
0 & 1
\end{array}\right) \bar{V}_{j}^{n+1}=\left(\begin{array}{cc}
2+R^{2} \delta^{2} & -h \\
1 & 0
\end{array}\right) \bar{V}_{j}^{n},
$$

where

$$
\begin{aligned}
k & =1+\gamma \frac{\Delta t}{2}-\frac{\beta \Delta t \delta^{2}}{2(\Delta r)^{2}}+m^{2} \frac{(\Delta t)^{2}}{2}, \quad \text { and } \\
h & =1-\gamma \frac{\Delta t}{2}+\frac{\beta \Delta t \delta^{2}}{2(\Delta r)^{2}}+m^{2} \frac{(\Delta t)^{2}}{2} .
\end{aligned}
$$

Denoting the Fourier transform of each $\bar{V}_{j}^{n}$ by $\hat{V}_{j}^{n}$, we get

$$
\hat{V}_{j}^{n+1}=\left(\begin{array}{cc}
\frac{2}{\hat{k}(\xi)}\left(1-2 R^{2} \sin ^{2} \frac{\xi}{2}\right) & -\frac{\hat{h}(\xi)}{\hat{k}(\xi)} \\
1 & 0
\end{array}\right) \hat{V}_{j}^{n},
$$

where 


$$
\begin{aligned}
& \hat{k}(\xi)=1+\gamma \frac{\Delta t}{2}+2 \frac{\beta \Delta t}{(\Delta r)^{2}} \sin ^{2} \frac{\xi}{2}+m^{2} \frac{(\Delta t)^{2}}{2}, \quad \text { and } \\
& \hat{h}(\xi)=1-\gamma \frac{\Delta t}{2}-2 \frac{\beta \Delta t}{(\Delta r)^{2}} \sin ^{2} \frac{\xi}{2}+m^{2} \frac{(\Delta t)^{2}}{2} .
\end{aligned}
$$

The matrix $A(\xi)$ multiplying $\hat{V}_{j}^{n}$ in the last vector equation is the amplification matrix of the problem. The characteristic equation $|\lambda I-A(\xi)|=0$ provides us with the eigenvalues of he amplification matrix, which are given by

$$
\lambda_{ \pm}=\frac{1-2 R^{2} \sin ^{2} \frac{\xi}{2} \pm \sqrt{\left(1-2 R^{2} \sin ^{2} \frac{\xi}{2}\right)-\hat{h}(\xi) \hat{k}(\xi)}}{\hat{k}(\xi)} .
$$

In particular, for $\xi=\pi$ the expressions for the eigenvalues of $A$ are

$$
\lambda_{ \pm}=\frac{1-2 R^{2} \pm \sqrt{\left(1-2 R^{2}\right)^{2}-\hat{h}(\pi) \hat{k}(\pi)}}{\hat{k}(\pi)} .
$$

Suppose for a moment that $1-2 R^{2}<-\hat{k}(\pi)$. If the radical in the expression for the eigenvalues of $A(\pi)$ is a pure real number then $\left|\lambda_{-}\right|>1$. So for every $n \in \mathbb{N}$, $\| A^{n}|| \geq\left|\lambda_{-}\right|^{n}$ grows faster than $K_{1}+n K_{2}$ for any constants $K_{1}$ and $K_{2}$. A similar situation happens when the radical is a pure imaginary number, except that in this case $|\cdot|$ represents the usual Euclidean norm in the field of complex numbers.

Summarizing, if $1-2 R^{2}<-\hat{k}(\pi)$ then scheme (4.8) is unstable order $n$. Therefore in order for our numeric method to be stable order $n$ it is necessary that $1-2 R^{2}>-\hat{k}(\pi)$, which is what we needed to prove. Convergence follows now from Lax theorem.

\subsection{Energy analysis}

As expected, the rate of change of the energy associated with the damped nonlinear Klein-Gordon equation is negative, provided that the $\beta$ and $\gamma$ are positive. In the present section, we formally prove this statement and describe a discrete scheme to approximate the energy at every time. Moreover, we demonstrate that the discrete rate of change of the energy yields a second-order approximation to the instantaneous rate of change.

\subsubsection{Continuous energy}

Let $\beta$ and $\gamma$ be positive numbers. For the sake of simplicity, suppose that $G$ is a continuously differentiable function in all $\mathbb{R}$. Then the differential equation in (4.1) can be rewritten as 


$$
\frac{\partial^{2} w}{\partial t^{2}}-\nabla^{2} w+m^{2} w+G^{\prime}(w)=-\gamma \frac{\partial w}{\partial t}+\beta \frac{\partial}{\partial t}\left(\nabla^{2} w\right) .
$$

The left-hand side of this equation represents the conservative part of our modified nonlinear Klein-Gordon equation, whereas the right-hand side contains its nonconservative contributions. We consider the Lagrangian of the nonlinear KleinGordon equation derived in Section 3.2,

$$
\mathcal{L}=\frac{1}{2}\left\{\left(\frac{\partial w}{\partial t}\right)^{2}-|\nabla w|^{2}-m^{2} w^{2}\right\}-G(w)
$$

From Lemma 2 it follows that $\mathcal{L}$ is the Lagrangian of the left-hand side of Equation (4.10). The following result is then straight-forward.

Lemma 5. The Hamiltonian associated with the modified nonlinear Klein-Gordon equation is given by

$$
\mathcal{H}=\frac{1}{2}\left\{\left(\frac{\partial w}{\partial t}\right)^{2}+|\nabla w|^{2}+m^{2} w^{2}\right\}+G(w) .
$$

ThEOREM 6. Let $D$ be a domain in $\mathbb{R}^{3}$ and assume that $\nabla u \cdot \hat{\mathrm{n}}=0$ in the boundary of $D$, where $\hat{\mathrm{n}}$ is the unit vector normal to the surface $\partial D$. Then the rate of change of the total energy

$$
E(t)=\iiint_{D}\left\{\frac{1}{2}\left(\frac{\partial w}{\partial t}\right)^{2}+\frac{1}{2}|\nabla w|^{2}+\frac{m^{2}}{2} w^{2}+G(w)\right\} d \bar{x}
$$

of the modified nonlinear Klein-Gordon equation is given by

$$
\frac{d E}{d t}=-\iiint_{D}\left\{\gamma\left(\frac{\partial w}{\partial t}\right)^{2}+\beta\left\|\nabla\left(\frac{\partial w}{\partial t}\right)\right\|^{2}\right\} d \bar{x} .
$$

Proof. Taking first the derivative on both sides of the energy equation, using then Equation (3.6) for $w$ and substituting next the modified nonlinear Klein-Gordon equation, we obtain that

$$
\begin{aligned}
\frac{d E}{d t} & =\iiint_{D} \frac{\partial w}{\partial t}\left\{\frac{\partial^{2} w}{\partial t^{2}}+m^{2} w+G^{\prime}(w)\right\} d \bar{x}+\frac{1}{2} \iiint_{D} \frac{\partial}{\partial t}|\nabla w|^{2} d \bar{x} \\
& =\iiint_{D} \frac{\partial w}{\partial t}\left\{\frac{\partial^{2} w}{\partial t^{2}}-\nabla^{2} w+m^{2} w+G^{\prime}(w)\right\} d \bar{x}+\iint_{\partial D} \frac{\partial w}{\partial t} \nabla w \cdot \hat{n} d \sigma \\
& =-\gamma \iiint_{D}\left(\frac{\partial w}{\partial t}\right)^{2} d \bar{x}+\beta \iiint_{D} \frac{\partial w}{\partial t} \nabla^{2}\left(\frac{\partial w}{\partial t}\right) d \bar{x}+\iint_{\partial D} \frac{\partial w}{\partial t} \nabla w \cdot \hat{\mathrm{n}} d \sigma
\end{aligned}
$$


On the other hand, from Green's first identity

$$
\iiint_{D} \frac{\partial w}{\partial t} \nabla^{2}\left(\frac{\partial w}{\partial t}\right) d \bar{x}=\iint_{\partial D} \frac{\partial w}{\partial t} \frac{\partial}{\partial t}(\nabla w \cdot \hat{\mathrm{n}}) d \sigma-\iiint_{D}\left\|\nabla\left(\frac{\partial w}{\partial t}\right)\right\|^{2} d \bar{x}
$$

The surface integrals in these equations are all equal to zero, whence the result follows.

The same conclusion can be obtained in the previous theorem if we drop the assumption of normality in the boundary of $D$ and suppose that $\phi$ and $\psi$ are smooth functions of compact support in $D$ which are small at infinity.

Assume that $w$ is a radially symmetric solution of the modified nonlinear Klein-Gordon equation, and let $D$ be the open sphere with center in the origin and radius $L$. Then the energy equation of the damped nonlinear Klein-Gordon equation can be written as Equation (3.5). The energy adopts the form $E(t)=4 \pi E_{0}(t)$ with $E_{0}$ given in Corollary 3.4, when we use the transformation $v(r, t)=r w(r, t)$.

Let $G$ be any continuously differentiable function. If $w$ is a radially symmetric solution of the modified nonlinear Klein-Gordon equation then the instantaneous rate of change of energy with respect to time in terms of $v$ is given by $E^{\prime}(t)=4 \pi E_{0}^{\prime}(t)$, where

$$
\frac{d E_{0}}{d t}(t)=-\int_{0}^{L}\left\{\gamma\left(\frac{\partial v}{\partial t}\right)^{2}+\beta\left(\frac{\partial^{2} v}{\partial r \partial t}-\frac{1}{r} \frac{\partial v}{\partial t}\right)^{2}\right\} d r
$$

For $\beta$ equal to zero, this last equation is in agreement with the instantaneous rate of change of the energy associated with the telegraph equation, proposed by Strauss in [37] as an exercise.

\subsubsection{Discrete energy}

The present section aims at deriving a discrete energy scheme with the property that the average rate of change of energy yields an approximation to the actual value of the corresponding instantaneous rate of change. In other words, for every $n=0,1, \ldots, N-1$ we wish to construct a numerical approximation $E_{n}^{0}$ of $E_{0}\left(t_{n}\right)$, in such a way that for every $n=1, \ldots, N-1$, the quotient $\left(E_{n}^{0}-E_{n-1}^{0}\right) / \Delta t$ approximates $E_{0}^{\prime}\left(t_{n}\right)$. Throughout this section $G^{\prime}(v)=v^{p}$.

Let $w(r, t)$ be a radially symmetric solution of the dissipative nonlinear Klein-Gordon equation in the sphere $D$ with center in the origin and radius $L$. Let $a<L$ and $T$ be positive numbers, and let $v(r, t)=r w(r, t)$. Construct partitions $0=r_{0}<r_{1}<\cdots<r_{M}=a$ and $0=t_{0}<t_{1}<\cdots<t_{N}=T$ of $[0, a]$ and $[0, T]$, respectively, into $M$ and $N$ subintervals of lengths $\Delta r=a / M$ and $\Delta t=T / N$, and denote the approximate value of $v\left(r_{j}, t_{n}\right)$ by $v_{j}^{n}$. We propose the scheme

$$
\begin{aligned}
\frac{E_{n}^{0}}{\Delta r}= & \frac{1}{2} \sum_{j=0}^{m-1}\left(\frac{v_{j}^{n+1}-v_{j}^{n}}{\Delta t}\right)^{2}+\frac{1}{2} \sum_{j=0}^{m-1}\left(\frac{v_{j+1}^{n+1}-v_{j}^{n+1}}{\Delta r}\right)\left(\frac{v_{j+1}^{n}-v_{j}^{n}}{\Delta r}\right) \\
& +\frac{1}{2} \sum_{j=0}^{m-1} \frac{\left(v_{j}^{n+1}\right)^{2}+\left(v_{j}^{n}\right)^{2}}{2}+\sum_{j=1}^{m-1} \frac{G\left(v_{j}^{n+1}\right)+G\left(v_{j}^{n}\right)}{2(j \Delta r)^{p-1}} .
\end{aligned}
$$


We proceed now to compute the difference $\left(E_{n}^{0}-E_{n-1}^{0}\right) / \Delta r$. Observe that for each $n=1, \ldots, N-1$ and $j=0,1, \ldots, m-1$,

$$
\begin{aligned}
\left(\frac{v_{j}^{n+1}-v_{j}^{n}}{\Delta t}\right)^{2}-\left(\frac{v_{j}^{n}-v_{j}^{n-1}}{\Delta t}\right)^{2} & =\left(\frac{v_{j}^{n+1}-2 v_{j}^{n}+v_{j}^{n-1}}{\Delta t^{2}}\right)\left(v_{j}^{n+1}-v_{j}^{n-1}\right), \\
\frac{\left(v_{j}^{n+1}\right)^{2}+\left(v_{j}^{n}\right)^{2}}{2}-\frac{\left(v_{j}^{n}\right)^{2}+\left(v_{j}^{n-1}\right)^{2}}{2} & =\left(\frac{v_{j}^{n+1}+v_{j}^{n-1}}{2}\right)\left(v_{j}^{n+1}-v_{j}^{n-1}\right), \quad \text { and } \\
\frac{G\left(v_{j}^{n+1}\right)+G\left(v_{j}^{n}\right)}{(j \Delta r)^{p-1}}-\frac{G\left(v_{j}^{n}\right)+G\left(v_{j}^{n-1}\right)}{(j \Delta r)^{p-1}} & =\left[\frac{G\left(v_{j}^{n+1}\right)-G\left(v_{j}^{n-1}\right)}{(j \Delta r)^{p-1}\left(v_{j}^{n+1}-v_{j}^{n-1}\right)}\right]\left(v_{j}^{n+1}-v_{j}^{n-1}\right) .
\end{aligned}
$$

The difference between the respective second terms of $E_{n}^{0} / \Delta r$ and $E_{n-1}^{0} / \Delta r$ presents some algebraic complications. We now state the way to express conveniently such difference. For every $n=1, \ldots, N-1$ and every $j=1, \ldots, m-1$,

$$
\begin{aligned}
& \left(\frac{v_{j+1}^{n+1}-v_{j}^{n+1}}{\Delta r}\right)\left(\frac{v_{j+1}^{n}-v_{j}^{n}}{\Delta r}\right)-\left(\frac{v_{j+1}^{n}-v_{j}^{n}}{\Delta r}\right)\left(\frac{v_{j+1}^{n-1}-v_{j}^{n-1}}{\Delta r}\right)= \\
& \quad-\left(\frac{\left.v_{j+1}^{n}-2 v_{j}^{n}+v_{j-1}^{n}\right)\left(v_{j}^{n+1}-v_{j}^{n-1}\right)+\frac{\left(v_{j+1}^{n}-v_{j}^{n}\right)\left(v_{j+1}^{n+1}-v_{j+1}^{n-1}\right)}{\Delta r^{2}}}{\Delta r^{2}}\right. \\
& \quad-\frac{\left(v_{j}^{n}-v_{j-1}^{n}\right)\left(v_{j}^{n+1}-v_{j}^{n-1}\right)}{\Delta r^{2}} .
\end{aligned}
$$

Notice that if $j$ equals 0 then the left-hand sides of all equations above are zero except for the last one which yields $v_{1}^{n}\left(v_{1}^{n+1}-v_{1}^{n-1}\right) / \Delta r^{2}$. These equations together with the discrete expression for the energy, scheme (4.8), the method of telescoping series, and the fact that $v_{0}^{n}$ and $v_{m}^{n}$ are all zero for $n=0,1, \ldots, N$, yield

$$
\begin{aligned}
\frac{E_{n}^{0}-E_{n-1}^{0}}{\Delta r}= & \sum_{j=1}^{m-1}\left(\frac{v_{j}^{n+1}-v_{j}^{n-1}}{2}\right)\left\{\frac{v_{j}^{n+1}-2 v_{j}^{n}+v_{j}^{n-1}}{(\Delta t)^{2}}-\frac{v_{j+1}^{n}-2 v_{j}^{n}+v_{j-1}^{n}}{(\Delta r)^{2}}\right. \\
& \left.+\frac{1}{2}\left[v_{j}^{n+1}+v_{j}^{n-1}\right]+\frac{1}{(j \Delta r)^{p-1}} \frac{G\left(v_{j}^{n+1}\right)-G\left(v_{j}^{n-1}\right)}{v_{j}^{n+1}-v_{j}^{n-1}}\right\} \\
& +\sum_{j=1}^{m-1}\left\{\frac{\left(v_{j+1}^{n}-v_{j}^{n}\right)\left(v_{j+1}^{n+1}-v_{j+1}^{n-1}\right)}{\Delta r^{2}}-\frac{\left(v_{j}^{n}-v_{j-1}^{n}\right)\left(v_{j}^{n+1}-v_{j}^{n-1}\right)}{\Delta r^{2}}\right\} \\
& +\frac{v_{1}^{n}\left(v_{1}^{n+1}-v_{1}^{n-1}\right)}{\Delta r^{2}} .
\end{aligned}
$$

Therefore 


$$
\begin{gathered}
\frac{E_{n}^{0}-E_{n-1}^{0}}{\Delta t}=-2 \beta \sum_{j=1}^{m-1}\left(\frac{v_{j}^{n+1}-v_{j}^{n-1}}{2 \Delta t}\right)\left(\frac{\left(v_{j}^{n+1}-v_{j}^{n-1}\right)-\left(v_{j-1}^{n+1}-v_{j-1}^{n-1}\right)}{2 \Delta r \Delta t}\right) \\
-\gamma \sum_{j=1}^{m-1}\left(\frac{v_{j}^{n+1}-v_{j}^{n-1}}{2 \Delta t}\right)^{2} \Delta r .
\end{gathered}
$$

If $\beta$ is equal to zero then the left-hand side of this last equation is a secondorder approximation for the instantaneous rate of change of the total energy of the modified nonlinear Klein-Gordon equation.

\subsection{Numerical results}

In this section we explore the numerical effects of external and internal damping. Throughout $G^{\prime}(w)=w^{p}$, for $p>1$ an odd number.

\subsubsection{External damping}

Let $\beta$ be equal to zero. Scheme (4.8) represents a generalization of the numerical method described in the previous chapter. Stability of the radially symmetric solutions for an initial-value problem involving a modified nonlinear Klein-Gordon equation with small $\gamma$ should lead us to solutions similar to those we would obtain for the same initial-value problem without damping term. Moreover, we would expect that the larger the value of the coefficient $\gamma$ the faster the solutions of the damped Klein-Gordon equation will diverge with respect to the undamped solution.

In order to confirm numerically these claims, let us consider initial-value problem (4.7) with $p=7, \gamma=0.5, \Delta r=\Delta t=0.002$, and initial data $\phi(r)=h(r)$ and $\psi(r)=h^{\prime}(r)+h(r) / r$, where

$$
h(r)= \begin{cases}5 \exp \left\{100\left[1-\frac{1}{1-(10 r-1)^{2}}\right]\right\}, & \text { if } 0 \leq r<0.2 \\ 0, & \text { if } 0.2 \leq r \leq 0.4\end{cases}
$$

We compare graphically the numerical solutions of the damped and the undamped nonlinear Klein-Gordon equations with the data recorded above, obtaining the graphs shown in Figure 4.2. Radially symmetric solutions to the undamped case are graphically represented by solid lines, whereas the solutions corresponding to the damped case are shown in dash-dotted lines. We observe that the solutions for the damped and the undamped case are close indeed. More precisely, let us consider the approximations $\bar{v}_{0}^{n}$ and $\bar{v}_{\gamma}^{n}$ to the undamped and damped nonlinear KleinGordon equations, respectively, for $n=0,20,40,60,80,100$. We compute the relative differences in the energy space using the formula

$$
\delta\left(\bar{v}_{\gamma}^{n}, \bar{v}_{0}^{n}\right)=\frac{\left\|\bar{v}_{\gamma}^{n}-\bar{v}_{0}^{n}\right\|_{2, \Delta x}}{\left\|\bar{v}_{0}^{n}\right\|_{2, \Delta x}} .
$$


$t=0$

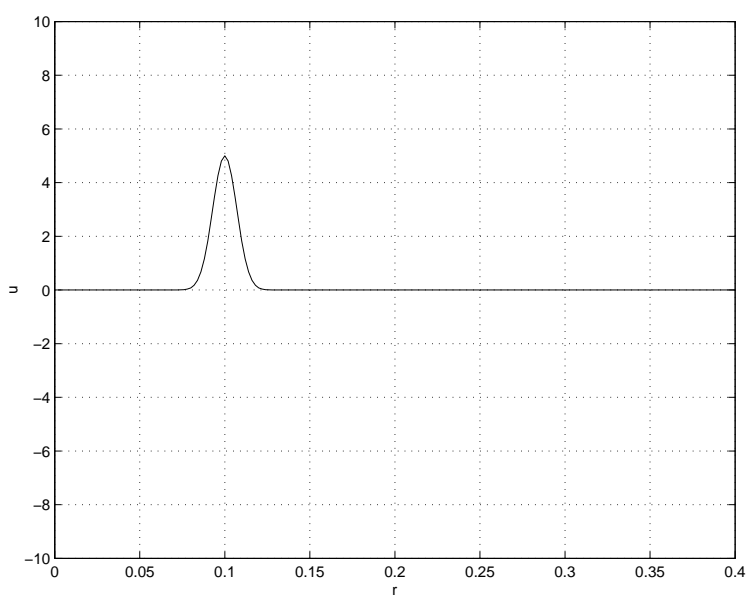

$t=0.08$

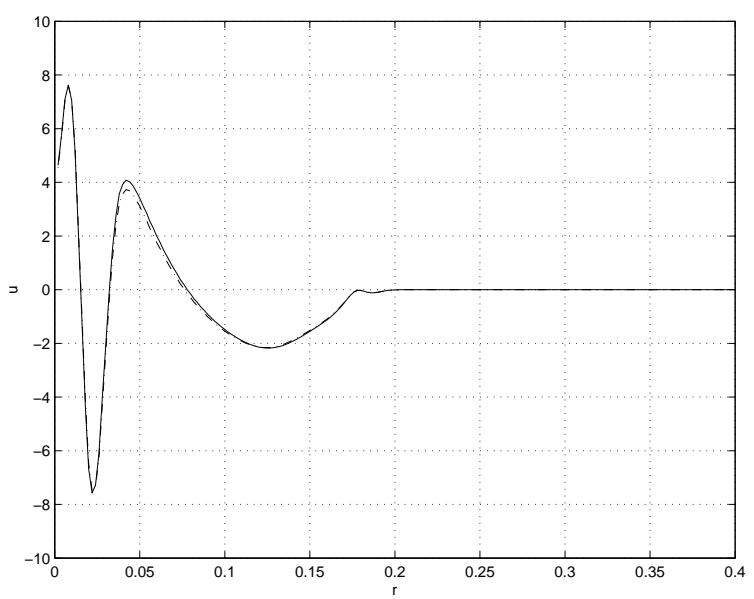

$t=0.16$

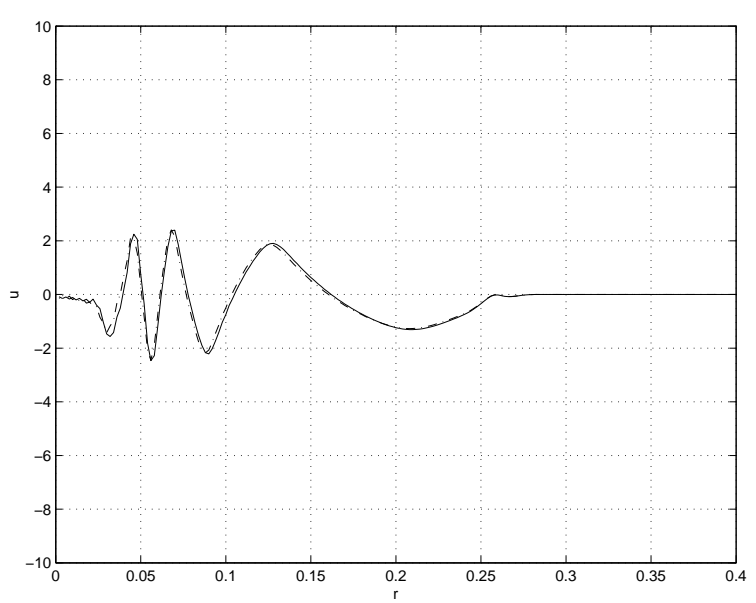

$t=0.04$

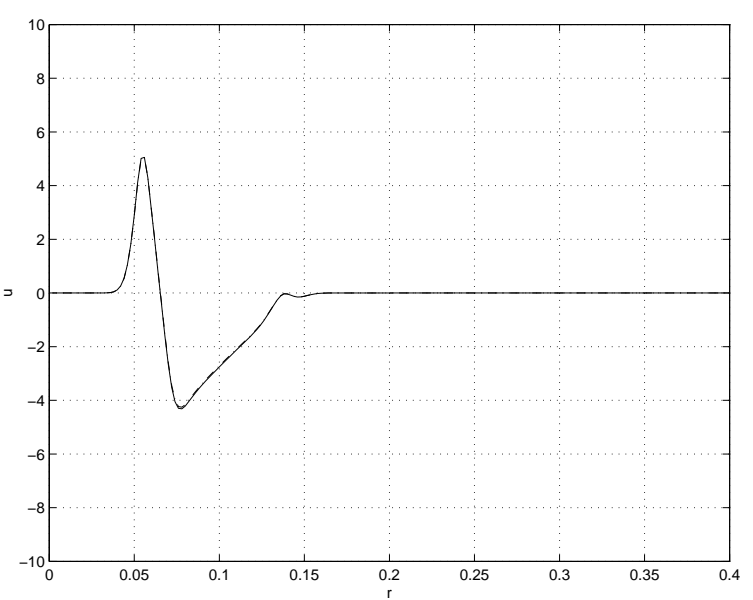

$t=0.12$

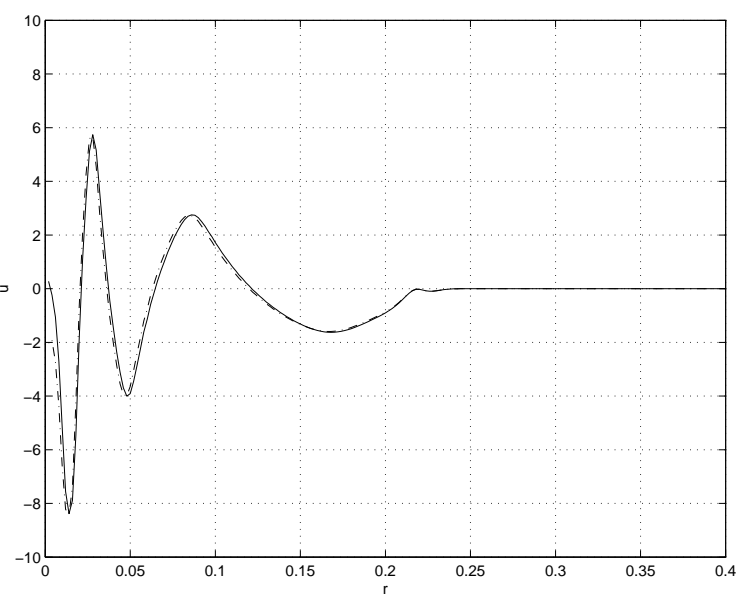

$t=0.2$

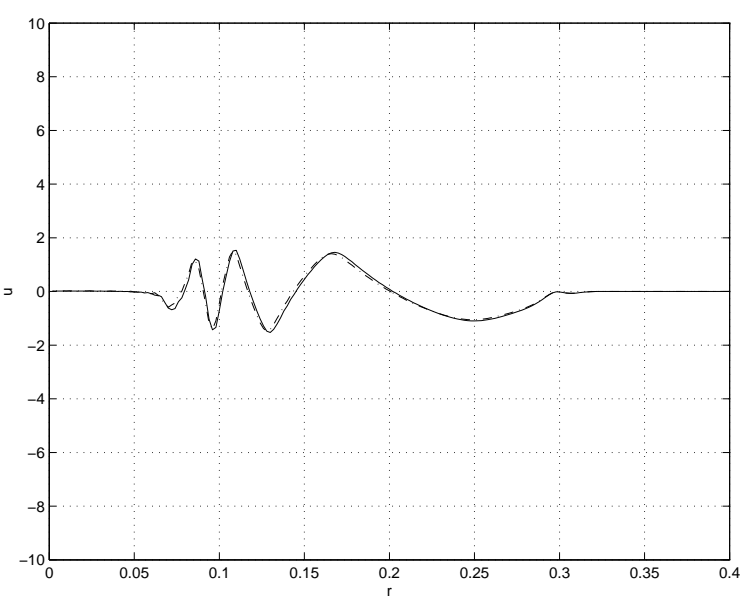

Figure 4.2: Approximate radial solutions at successive times of the undamped (solid line) and the damped (dash-dotted line) nonlinear Klein-Gordon equations with $\gamma=0.5$, $G^{\prime}(u)=u^{7}$, initial data $\phi(r)=h(r), \psi(r)=h^{\prime}(r)+h(r) / r$, and boundary condition $u(0.4, t)=0$. 
$t=0$

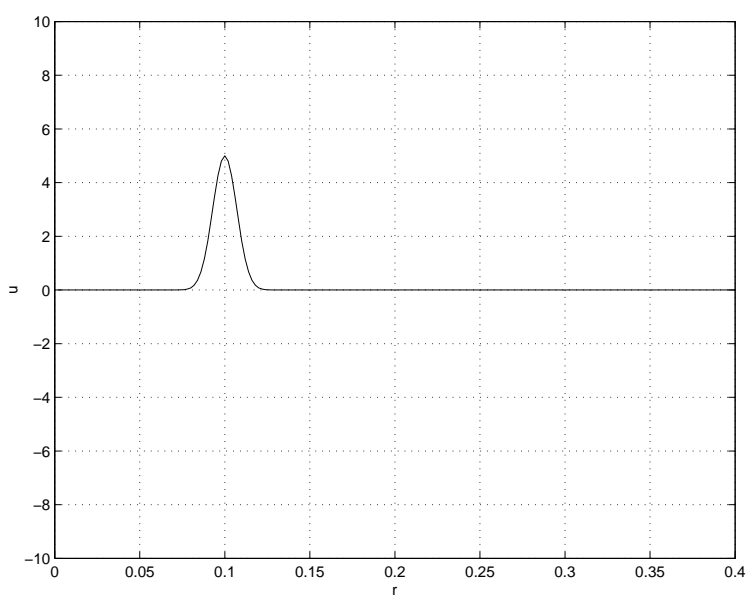

$t=0.08$

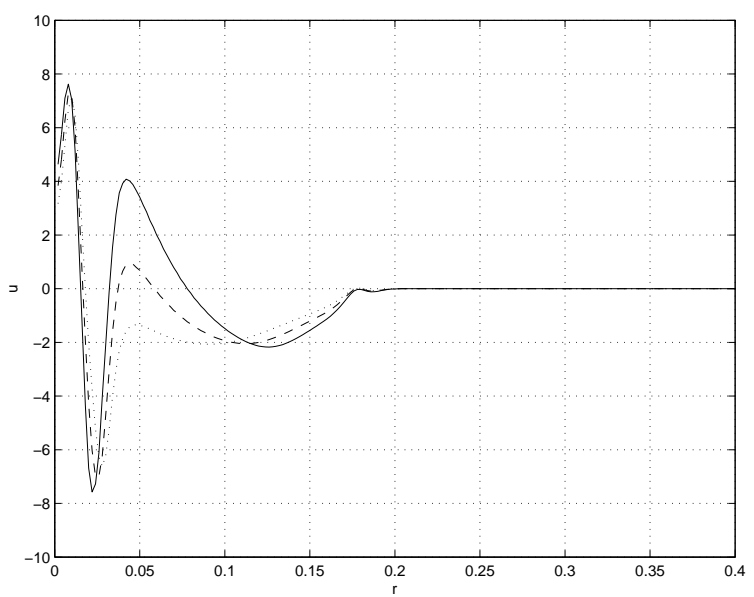

$t=0.16$

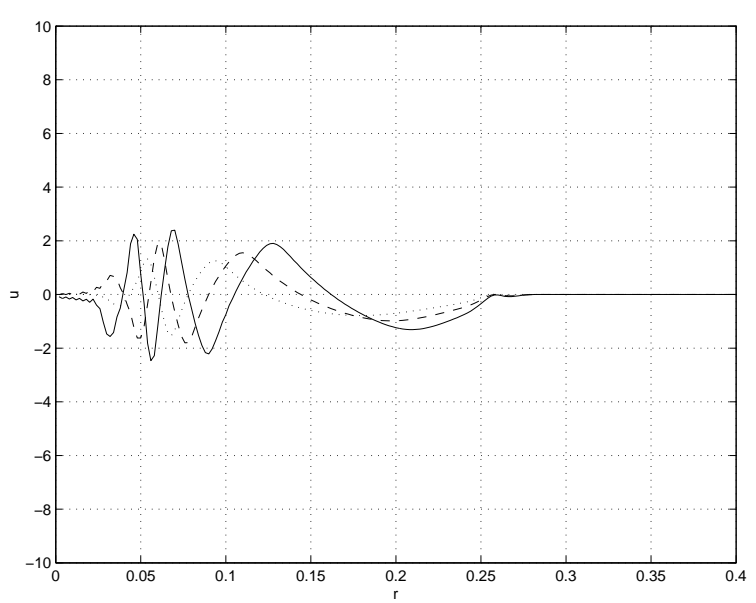

$t=0.04$

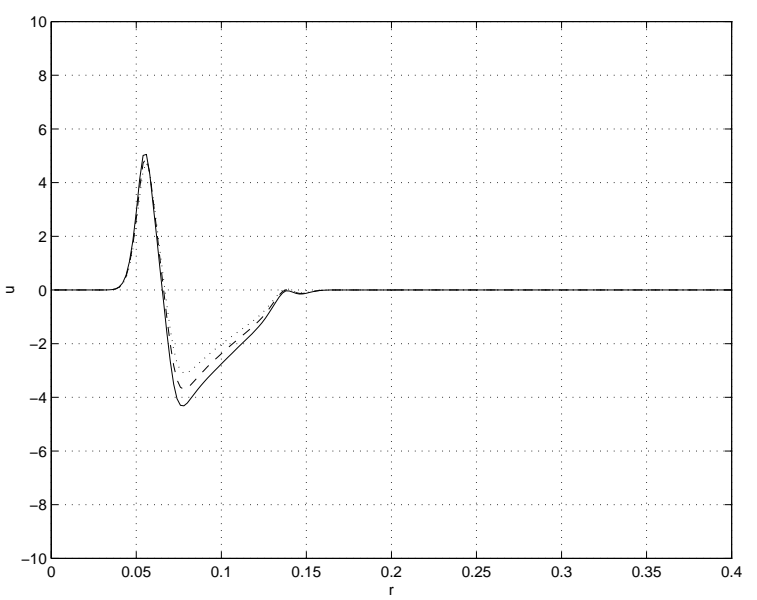

$t=0.12$

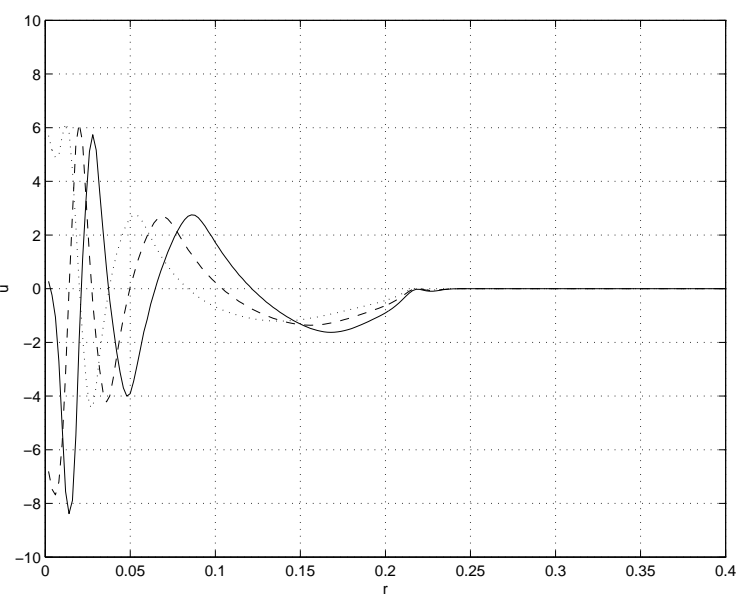

$t=0.2$

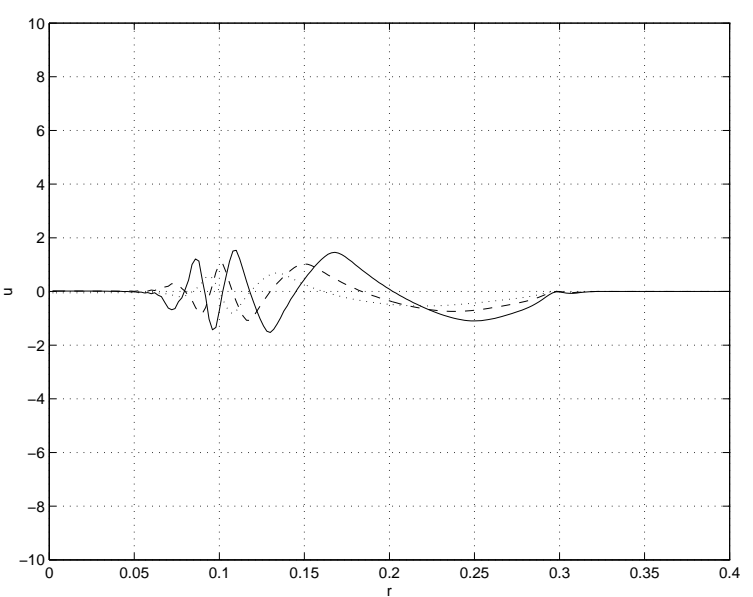

Figure 4.3: Approximate radial solutions at successive times of the undamped (solid line) and the damped nonlinear Klein-Gordon equation with $\gamma=5$ (dashed line) and $\gamma=10$ (dotted line), nonlinear term $G^{\prime}(u)=u^{7}$, initial data $\phi(r)=h(r)$, $\psi(r)=h^{\prime}(r)+h(r) / r$, and boundary condition $u(0.4, t)=0$. 

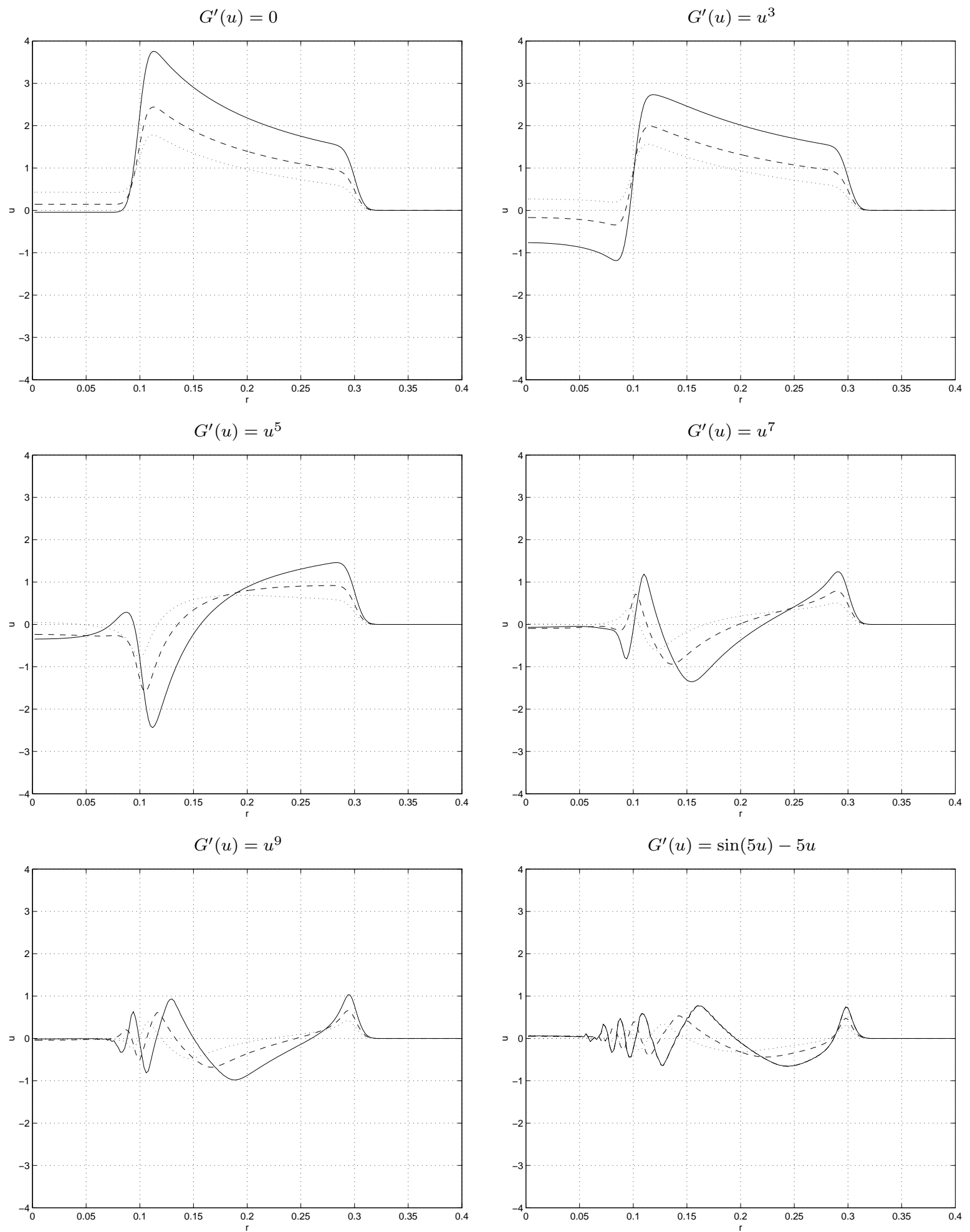

FiguRE 4.4: Approximate radial solutions for the damped nonlinear Klein-Gordon equation with nonlinear function $G^{\prime}(u)$ at time $t=0.2$, for initial data $\phi(r)=0$ and $\psi(r)=$ $100 h(r)$, boundary condition $u(0.4, t)=0$, and damping coefficients $\gamma=0$ (solid line), $\gamma=5$ (dashed line), and $\gamma=10$ (dotted line), respectively. 
$t=0$

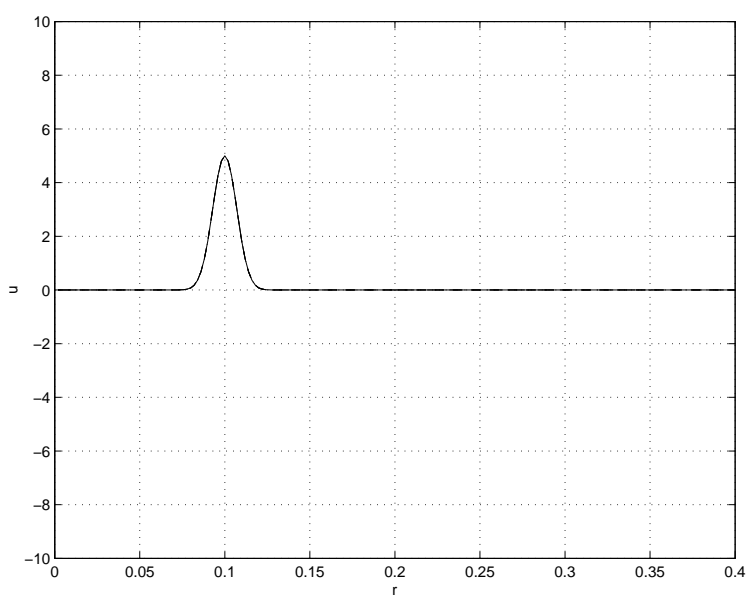

$t=0.08$

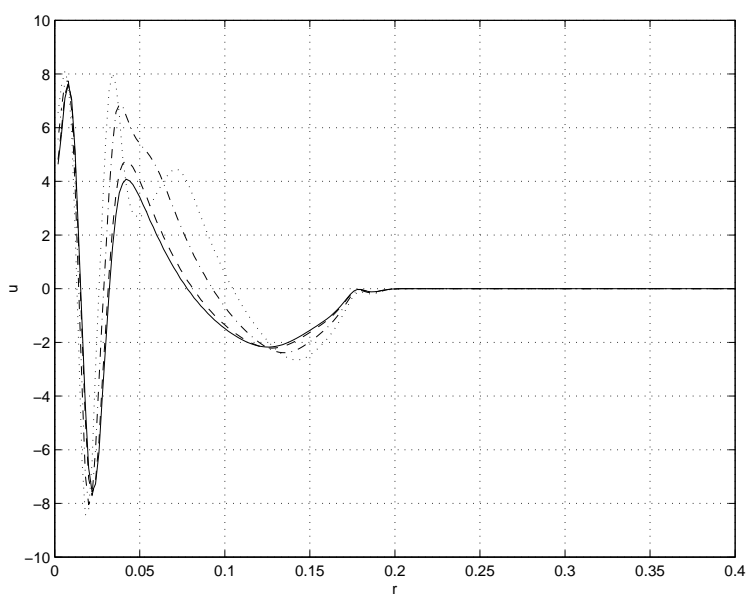

$t=0.16$

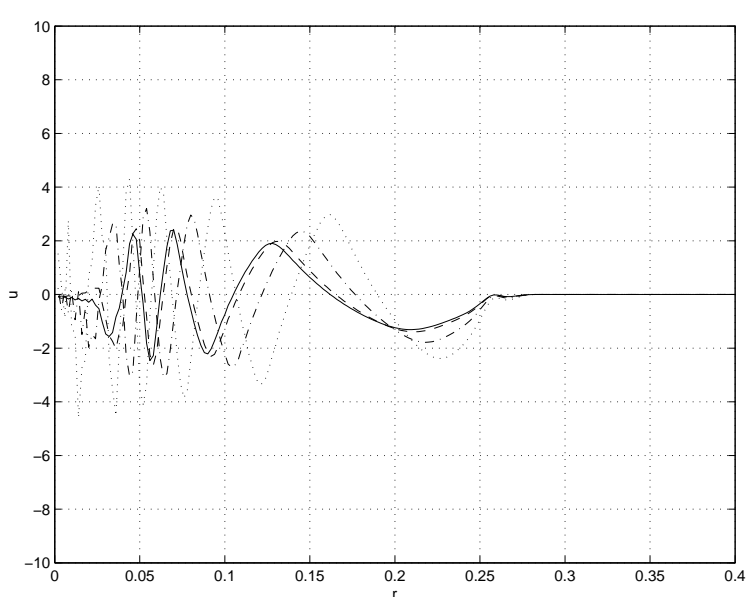

$t=0.04$

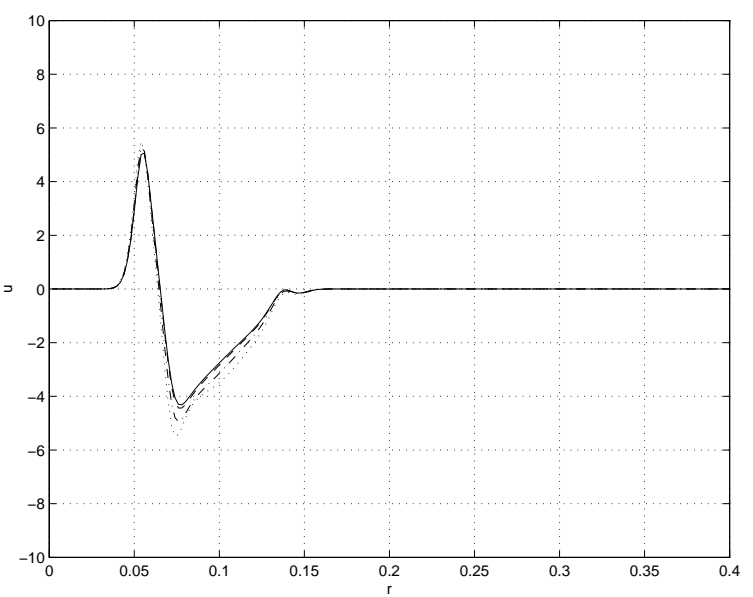

$t=0.12$

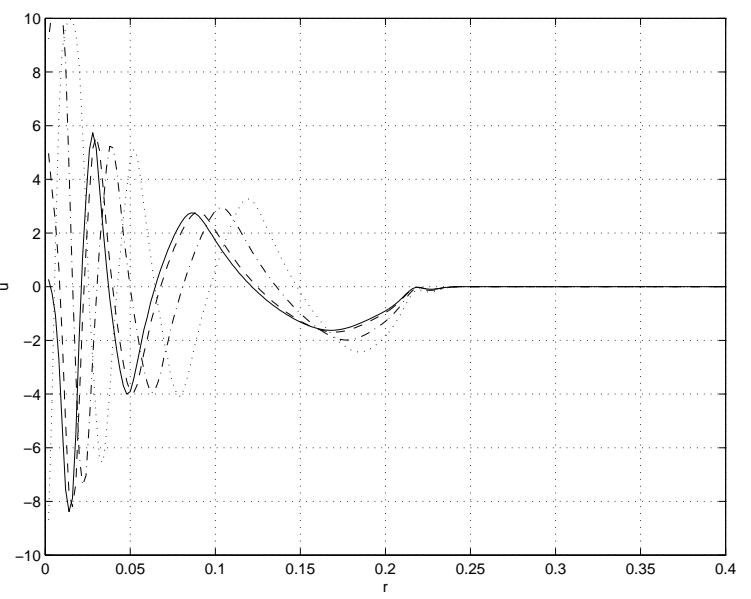

$t=0.2$

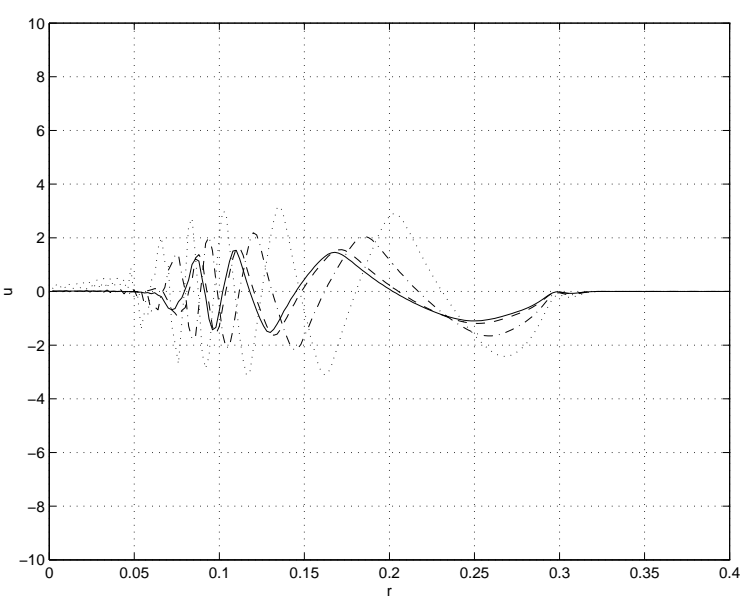

FigurE 4.5: Approximate radial solutions at successive times of the undamped (solid) and the damped nonlinear Klein-Gordon equation with $\gamma=-1$ (dashed), $\gamma=-5$ (dash-dotted), and $\gamma=-10$ (dotted), nonlinear term $G^{\prime}(u)=u^{7}$, and initial data $\phi(r)=h(r), \psi(r)=h^{\prime}(r)+h(r) / r$, and boundary condition $u(0.4, t)=0$. 


\begin{tabular}{|c|c|c|c|c|c|}
\hline Time step & \multicolumn{5}{|c|}{ Relative differences } \\
\cline { 2 - 6 }$n$ & $\gamma=0.1$ & $\gamma=0.5$ & $\gamma=1$ & $\gamma=5$ & $\gamma=10$ \\
\hline \hline 0 & 0.0000 & 0.0000 & 0.0000 & 0.0000 & 0.0000 \\
\hline 20 & 0.0028 & 0.0142 & 0.0283 & 0.1395 & 0.2693 \\
\hline 40 & 0.0103 & 0.0509 & 0.1006 & 0.4491 & 0.7706 \\
\hline 60 & 0.0167 & 0.0821 & 0.1611 & 0.6579 & 0.9573 \\
\hline 80 & 0.0192 & 0.0942 & 0.1836 & 0.6954 & 0.9387 \\
\hline 100 & 0.0200 & 0.0977 & 0.1896 & 0.6994 & 0.9308 \\
\hline
\end{tabular}

TABLE 4.2: Table of relative differences of solutions of the damped nonlinear Klein-Gordon equation (nonlinear term $G^{\prime}(w)=w^{7}$ and varying damping coefficient $\gamma$ ) with respect to the corresponding undamped solution at different time steps, using $\Delta r=$ $\Delta t=0.002$ and initial data $\phi(r)=h(r), \psi(r)=h^{\prime}(r)+h(r) / r$.

Relative differences for values of $\gamma$ equal to $0.1,0.5,1,5$, and 10 are shown in Table 4.2. We conclude that the larger the value of $\gamma$ the larger the relative difference of $\bar{v}_{\gamma}^{n}$ with respect to $\bar{v}_{0}^{n}$. To verify this claim graphically, we appeal to Figure 4.3, which compares radially symmetric numerical solution of our initial-value problem for values of $\gamma$ equal to 0,5 , and 10 . The graphs and the results included in the table suggest that our method is stable for our choice of time and space steps.

In Figure 4.4 we have printed numerical solutions of the modified nonlinear Klein-Gordon equation with $\gamma=0,5$ and 10, nonlinear terms $G^{\prime}(u)=0, u^{3}, u^{5}, u^{7}, u^{9}$, and $\sinh (5 u)-5 u$, initial data $\phi(r)=0$ and $\psi(r)=100 h(r)$, and $\Delta r=\Delta t=0.002$. This figure together with the information on the relative differences of these solutions with respect to the undamped case contained in Table 4.3, indeed provide us with support to establish the dependence of the relative differences over the parameter $\gamma$.

Notice that the choice of $\gamma$ is reflected in the amplitude of the solutions. More accurately, the larger the value of $\gamma$ the smaller the value of the amplitude will become eventually in time after all the oscillations have been created. To verify this claim, we appeal to Figure 4.3 for $t=0.2$. The same observation can be more easily drawn from the graphs in Figure 4.4. For negative values of $\gamma$ we observe again this dependence between amplitude and $\gamma$ : The more negative the value of $\gamma$ the larger

\begin{tabular}{|c|c|c|c|c|c|}
\hline $\begin{array}{c}\text { Nonlinear Term } \\
G^{\prime}(w)\end{array}$ & \multicolumn{5}{|c|}{ Relative differences } \\
\cline { 2 - 6 } & $\gamma=0.1$ & $\gamma=0.5$ & $\gamma=1$ & $\gamma=5$ & $\gamma=10$ \\
\hline \hline 0 & 0.0098 & 0.0478 & 0.0923 & 0.3642 & 0.5631 \\
\hline$w^{3}$ & 0.0097 & 0.0477 & 0.0929 & 0.3528 & 0.5554 \\
\hline$w^{5}$ & 0.0137 & 0.0665 & 0.1287 & 0.4024 & 0.6418 \\
\hline$w^{7}$ & 0.0171 & 0.0833 & 0.1618 & 0.5068 & 0.7819 \\
\hline$w^{9}$ & 0.0204 & 0.0999 & 0.1728 & 0.5736 & 0.8488 \\
\hline $\sinh (5 w)-5 w$ & 0.0263 & 0.1377 & 0.2518 & 0.6284 & 0.8813 \\
\hline
\end{tabular}

TABLE 4.3: Table of relative differences of solutions of the damped nonlinear Klein-Gordon equation (varying nonlinear term $G^{\prime}(w)$ and varying damping coefficient $\gamma$ ) with respect to the corresponding undamped solution at time $t=0.2$, using $\Delta r=\Delta t=$ 0.002 and common initial data. 
the value of the amplitude. This observation can be corroborated after looking at Figure 4.5 for time $t=0.2$.

To close this section we must mention that the rate of change of the energy with respect to time is negative for positive values of $\gamma$, as expected from the theory. For positive values of $\gamma$ the rate of change of energy is positive, and if $\gamma$ is equal to 0 the rate of change is likewise equal to zero. Experimental results show us that small values of $\gamma$ go with small values of the rate of change of energy, whereas large values of $\gamma$ yield large values of the rate of change of energy.

\subsubsection{Internal damping}

Our main goal in this section is to study the effect of internal damping in scheme (4.8). We first consider the case when $\gamma$ is zero and study the behavior of solutions for small values of $\beta$. Since our method is a generalization of the method presented in Chapter 3, we expect that small values of $\beta$ will yield solutions very similar to those we would get for the undamped case.

\section{The externally undamped case}

Let us study the case $\gamma=0$ and $p=7$. Let $\Delta r=\Delta t=0.002$, and let the initial data be $\phi(r)=h(r)$ and $\psi(r)=h^{\prime}(r)+h(r) / r$, where the function $h$ is given as in the previous section. From the graphical results displayed in Figure 4.6 we infer that small values of $\beta$ lead to slight changes in the behavior of the solution of the modified nonlinear Klein-Gordon equation with respect to the undamped case.

To be more precise in this matter, we appeal again to the relative difference of damped solutions on the nonlinear Klein-Gordon equation with respect to the corresponding undamped solution under the $\ell_{2, \Delta x}$ norm proposed in the previous section. Table 4.4 records these relative differences for various values of $\beta$. As expected, we notice that the relative differences tend to increase when the value of $\beta$ increases.

\begin{tabular}{|c|c|c|c|c|c|}
\hline Time step & \multicolumn{5}{|c|}{ Relative differences } \\
\cline { 2 - 6 }$n$ & $\beta=10^{-6}$ & $\beta=10^{-5}$ & $\beta=10^{-4}$ & $\beta=0.0005$ & $\beta=0.001$ \\
\hline \hline 0 & 0.0000 & 0.0000 & 0.0000 & 0.0000 & 0.0000 \\
\hline 20 & 0.0007 & 0.0056 & 0.0524 & 0.2229 & 0.3779 \\
\hline 40 & 0.0034 & 0.0304 & 0.2648 & 0.8282 & 1.1467 \\
\hline 60 & 0.0563 & 0.0996 & 0.8819 & 1.6798 & 1.1486 \\
\hline 80 & 0.1881 & 0.1738 & 0.6767 & 1.1556 & 1.2242 \\
\hline 100 & 0.1362 & 0.1164 & 0.5442 & 1.0439 & 1.1637 \\
\hline
\end{tabular}

TABLE 4.4: Table of relative differences of solutions of the damped nonlinear Klein-Gordon equation (nonlinear term $G^{\prime}(w)=w^{7}$ and varying damping coefficient $\beta$ ) with respect to the corresponding undamped solution at different time steps, using $\Delta r=$ $\Delta t=0.002$ and initial data $\phi(r)=h(r), \psi(r)=h^{\prime}(r)+h(r) / r$.

In Figure 4.7 we have plotted numerical solutions of the nonlinear KleinGordon equation for $\gamma$ equal to zero and different positive and small values of $\beta$. We 
observe again that small values of $\beta$ yield solutions which are close to the solution of the undamped Klein-Gordon equation.

Throughout our experiments we have inevitably observed that the value of the discrete energy is decreasing at an apparently exponential rate. Moreover, we have noticed that the larger the value of $\beta$ the larger the absolute value of the rate of change of the energy with respect to time. Furthermore, it seems that the limit of the value of the energy when time increases to infinity is equal to zero.

Another interesting observation is the fact that the value of numerical solutions near the origin tend to increase with $\beta$. That can be easily established from Figures 4.7 and 4.8. In the former we have plotted numerical solutions to the modified nonlinear Klein-Gordon equation with nonlinear term $G^{\prime}(u)=u^{7}$, initial conditions $\phi(r)=h(r)$ and $\psi(r)=h^{\prime}(r)+h(r) / r$, and boundary condition $u(0.4, t)=0$ for every $t$; our space and time steps were chosen as $\Delta r=\Delta t=0.002$. This figure shows that the solutions for $\beta=0.005$ tend to increase near the origin almost immediately after start. The latter figure shows numerical solutions of the dissipative nonlinear Klein-Gordon equation for different nonlinear terms $G^{\prime}(u)=u^{3}, u^{5}, u^{7}$ at time $t=0.2$. The first column shows numerical solutions for the initial data set $\phi(r)=0$ and $\psi(r)=100 h(r)$, and the second shows the numerical solutions corresponding to the conditions $\phi(r)=h(r)$ and $\psi(r)=0$.

We must remark that we ran our numeric scheme for negative values of $\beta$. For values close to zero the numeric solutions we obtained were close to the numeric solutions of the undamped case. At the same time, we observed that the value of the energy tended to increase at a seemingly exponential rate. As expected, the solutions for large negative values of $\beta$ blew up in finite time.

\section{The externally damped case}

We approximate the solution to our modified nonlinear Klein-Gordon equation at $t=0.2$ for $\gamma=5$, using $G^{\prime}(u)=0, u, u^{3}, u^{5}, u^{7}$, and $u^{9}$ as nonlinear terms, initial data $\phi(r)=0$ and $\psi(r)=100 h(r)$, and $\Delta r=0.002$ and $\Delta t=0.001$. Different values of $\beta$ were chosen: $\beta=0,0.0006$, and 0.005 . The results - presented in Figure 4.9 - make it clear that small values of $\beta$ correspond with small changes in the solution of the nonlinear Klein-Gordon equation with respect to the internally undamped case; this suggests continuity of our method with respect to the parameter $\beta$.

As in the preceding section, solutions to the nonlinear Klein-Gordon equation tend to increase in time around the origin. Moreover, larger values of $\beta$ yield a faster growth of the numeric solution for values of $r$ close to zero. On the other hand, for values of $r$ far away from zero we notice a decay in the amplitude of the numeric solutions. We ran our method for larger periods of time and notice that these observations were consistently true (graphical results not included). We must mention that the values of energy were always decreasing. For larger values of $\beta$ the rate of decay was noticeably larger, always following an apparent exponential pattern. Among other things, for positive values of $\beta$ we observed that the energy tends to converge to zero as time goes to infinity. On the other hand, for negative values of $\beta$ the value of the energy rapidly blows up to infinity and the solutions tend to diverge in finite time. 
$t=0$

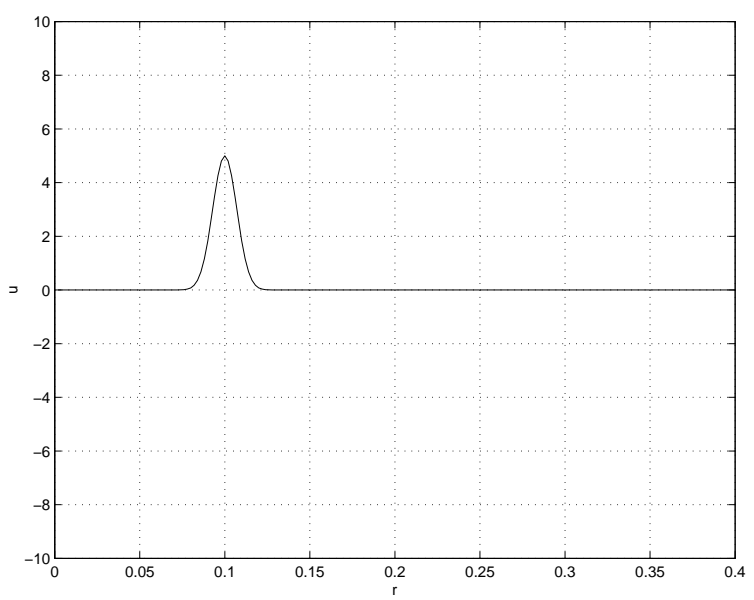

$t=0.08$

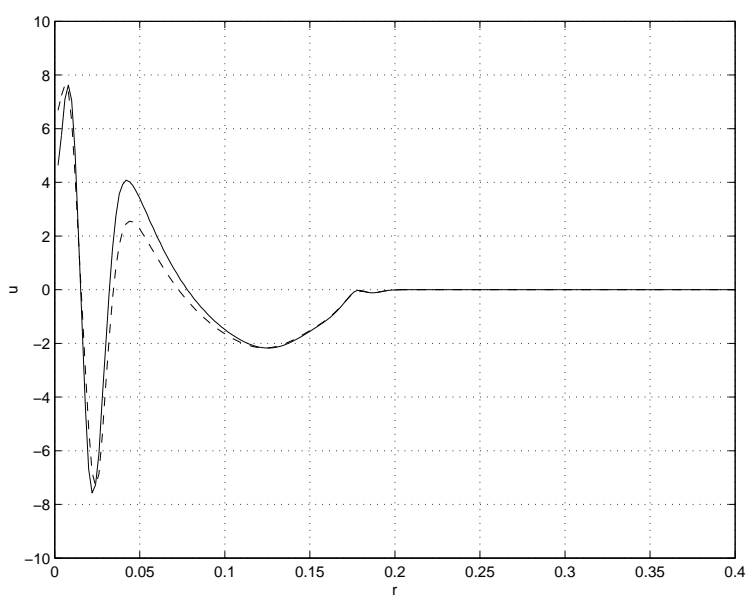

$t=0.16$

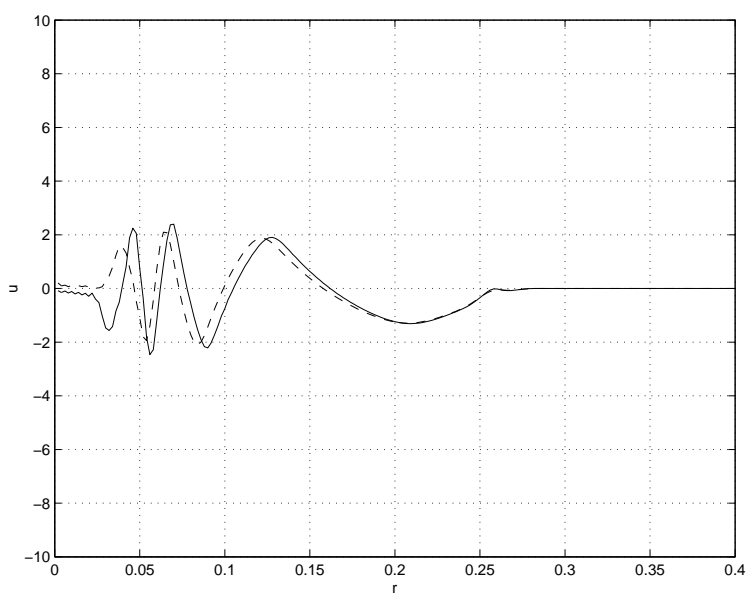

$t=0.04$

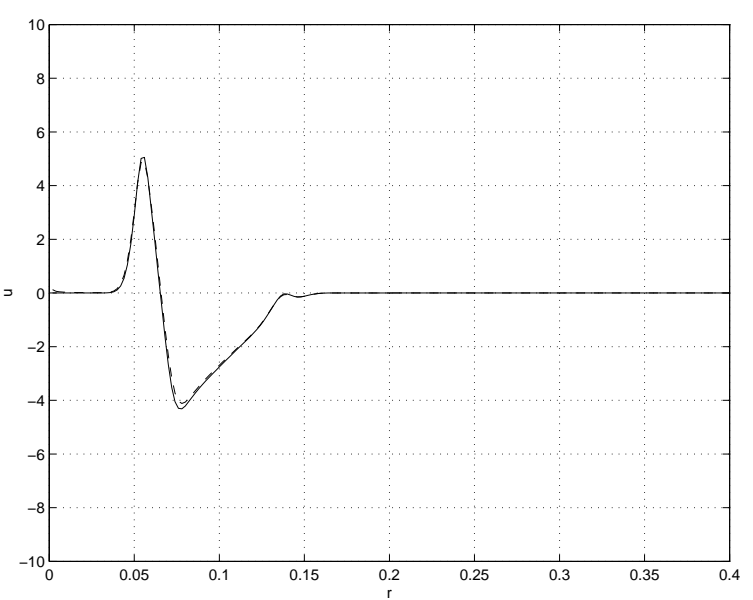

$t=0.12$

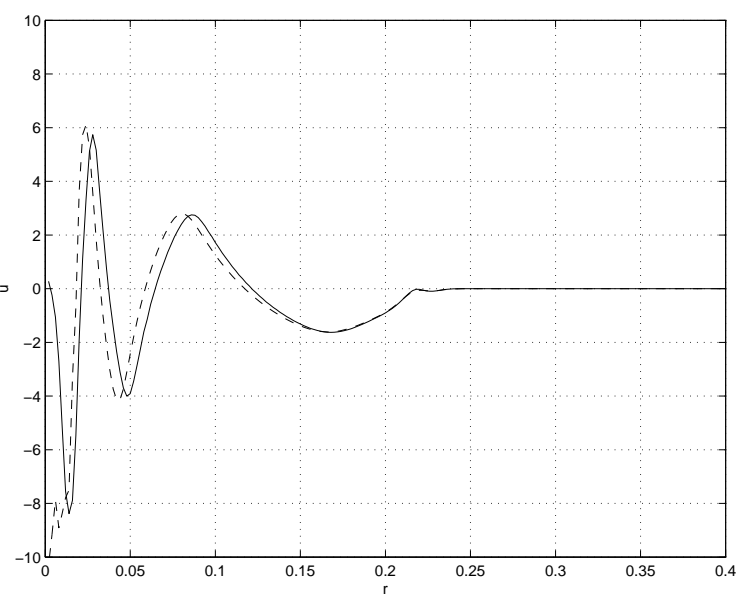

$t=0.2$

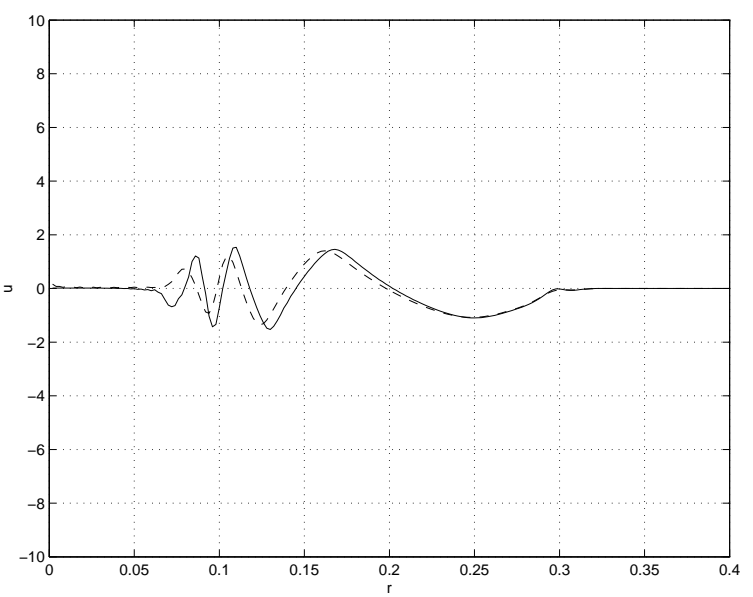

Figure 4.6: Approximate radial solutions at successive times of the undamped (solid line) and the damped (dash-dotted line) nonlinear Klein-Gordon equations with $\beta=0.0001, G^{\prime}(u)=u^{7}$, initial and boundary conditions $\phi(r)=h(r)$ and $\psi(r)=$ $h^{\prime}(r)+h(r) / r$, and $u(0.4, t)=0$, respectively. 
$t=0$

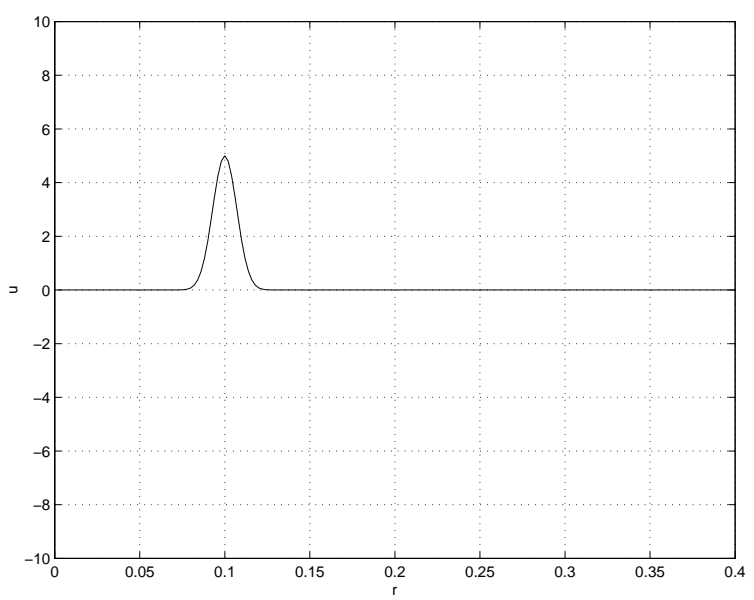

$t=0.08$

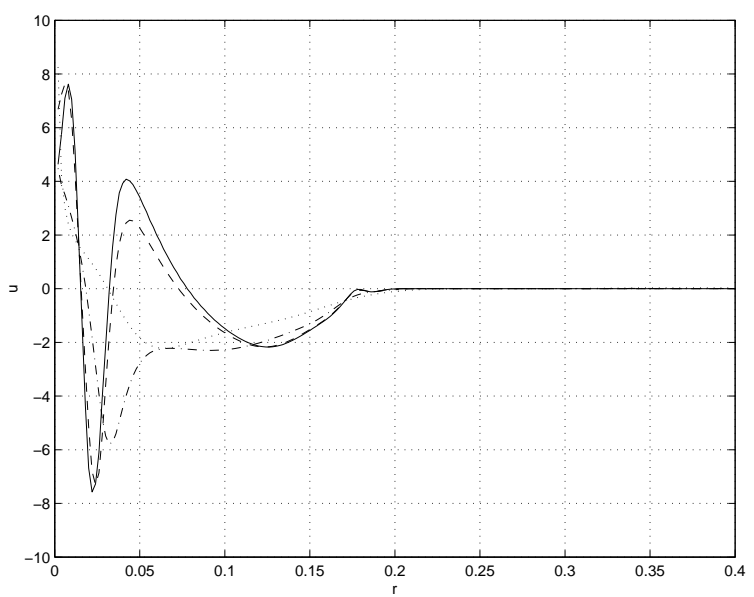

$t=0.16$

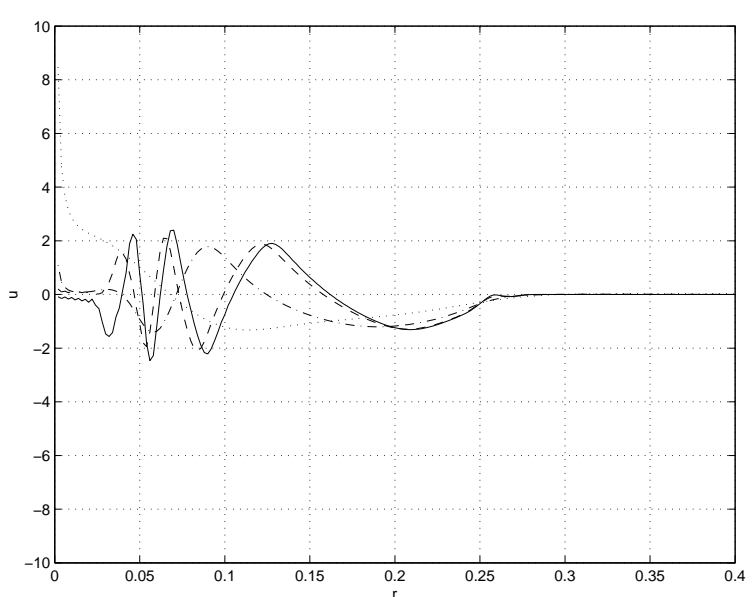

$t=0.04$

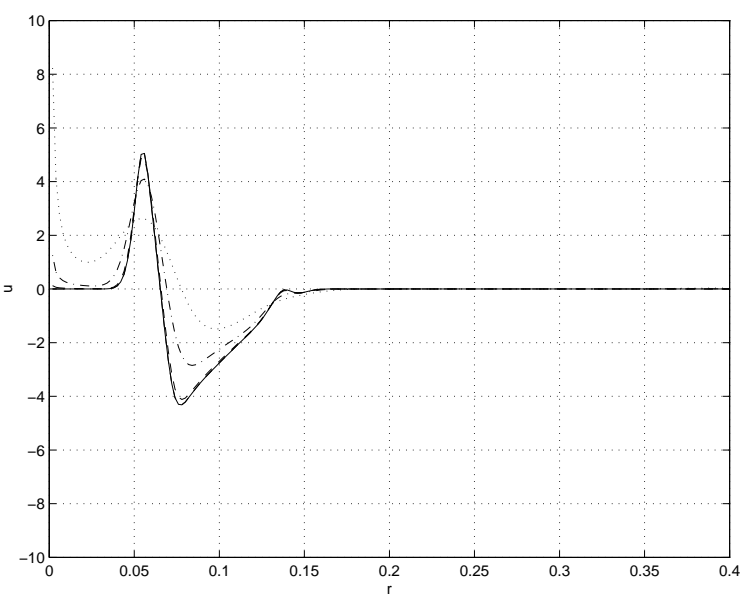

$t=0.12$

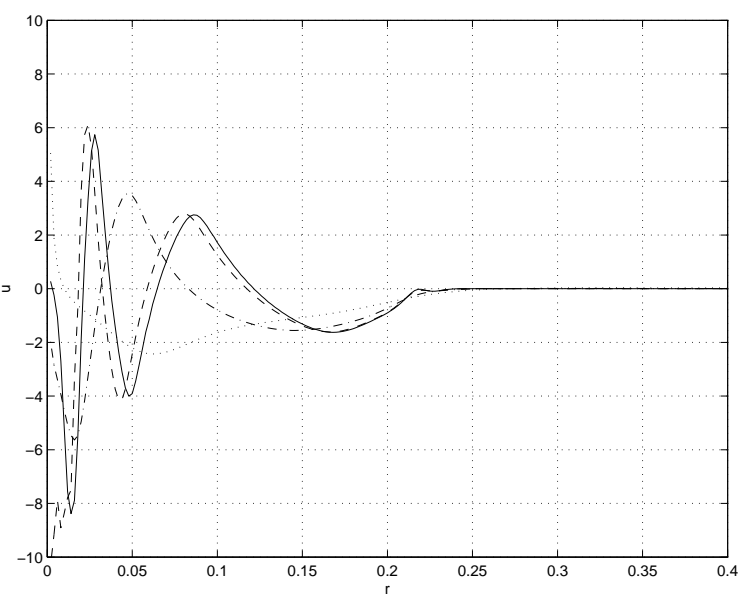

$t=0.2$

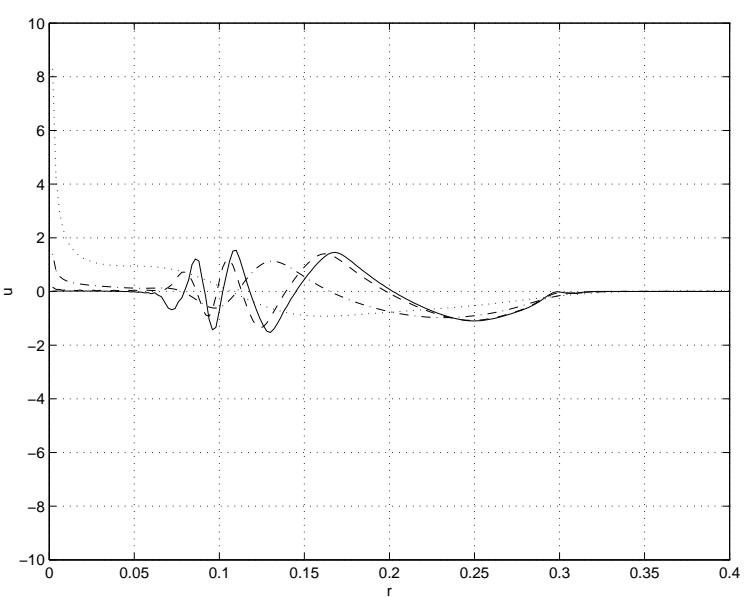

Figure 4.7: Approximate radial solutions at successive times of the undamped (solid) and the damped nonlinear Klein-Gordon equation with $\beta=0.0001$ (dashed), $\beta=0.001$ (dash-dotted) and $\beta=0.005$, nonlinear term $G^{\prime}(u)=u^{7}$, initial data $\phi(r)=h(r)$ and $\psi(r)=h^{\prime}(r)+h(r) / r$, and boundary condition $u(0.4, t)=0$. 

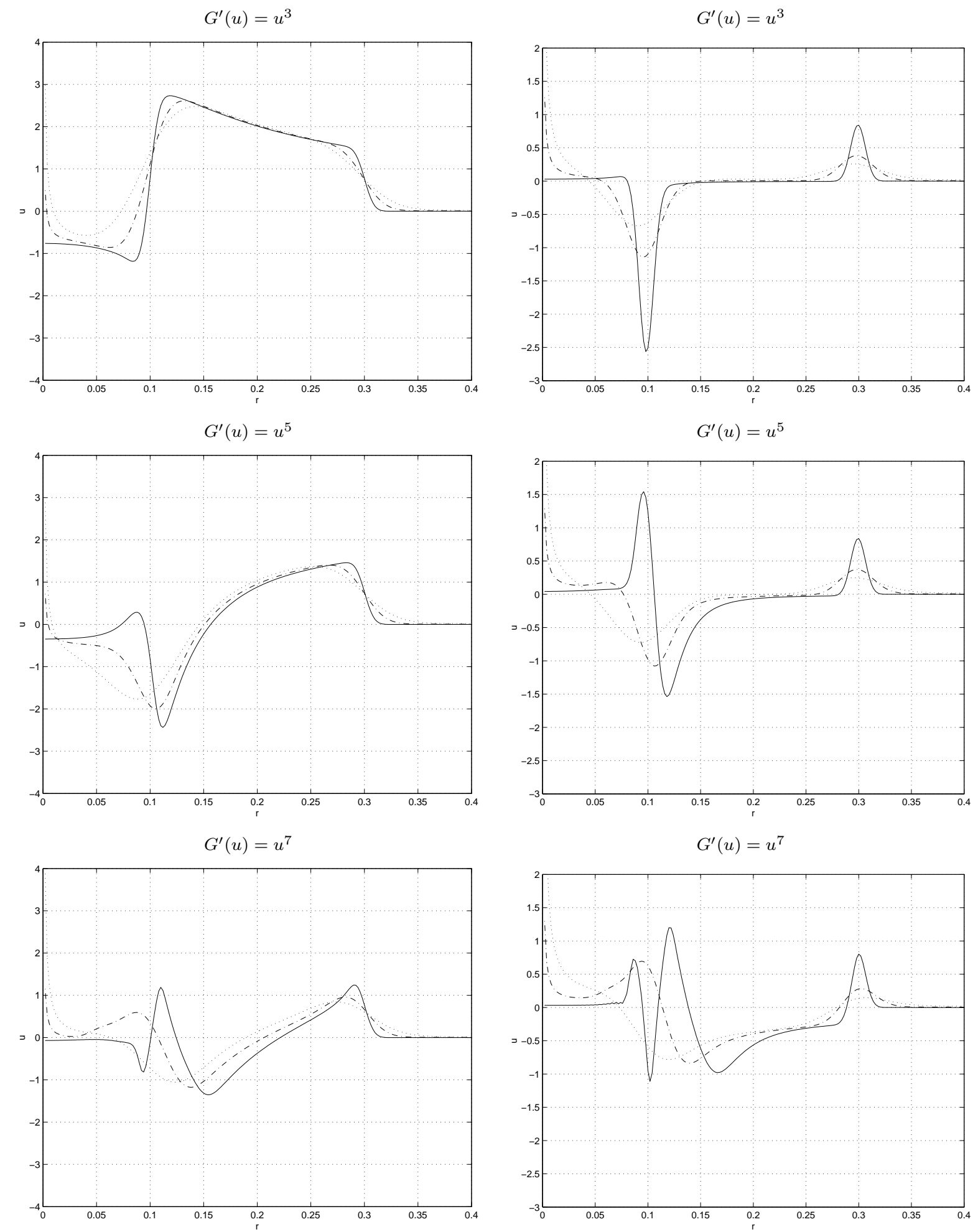

Figure 4.8: Approximate radial solutions for the Klein-Gordon equation with nonlinearity $G^{\prime}(u)$ at $t=0.2$ for initial data $\phi(r)=0, \psi(r)=100 h(r)$ (left column) and $\phi(r)=h(r), \psi(r)=0$ (right column), boundary condition $u(0.4, t)=0$, and $\beta=0$ (solid), $\beta=0.001$ (dashed), and $\beta=0.003$ (dotted). 

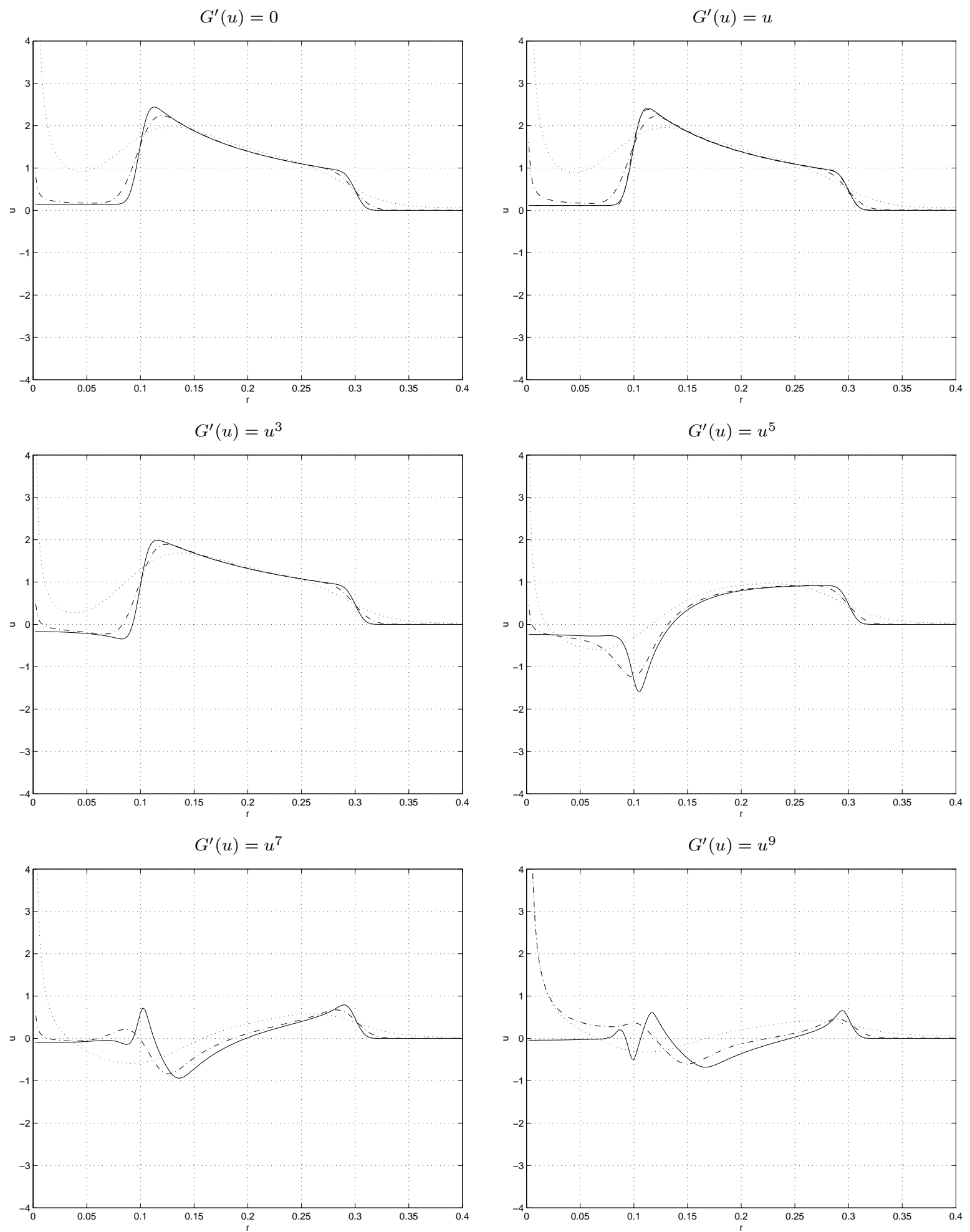

Figure 4.9: Approximate radial solutions for the Klein-Gordon equation with nonlinearity $G^{\prime}(u)$ and $\gamma=5$ at time $t=0.2$, for initial data $\phi(r)=0$ and $\psi(r)=100 h(r)$, boundary condition $u(0.4, t)=0$, and damping coefficients $\beta=0$ (solid line), $\beta=$ 0.0006 (dashed line), and $\beta=0.005$ (dotted line). 
$G^{\prime}(u)=u^{3}$

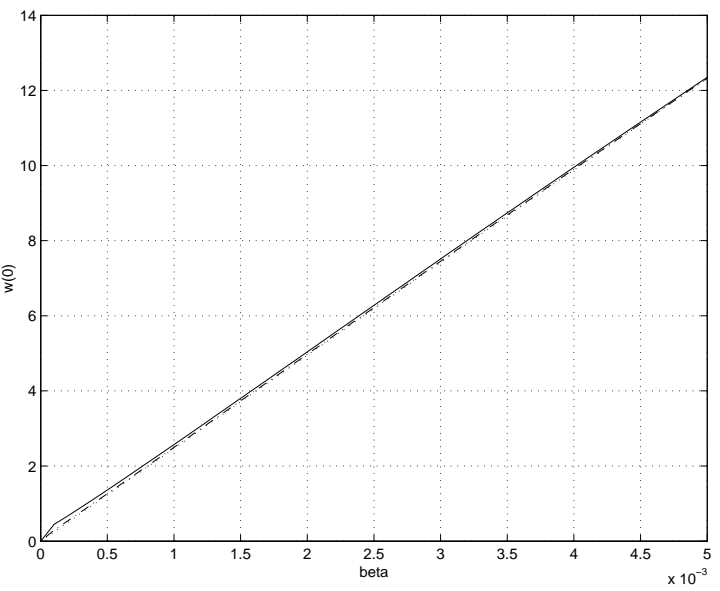

$G^{\prime}(u)=u^{5}$

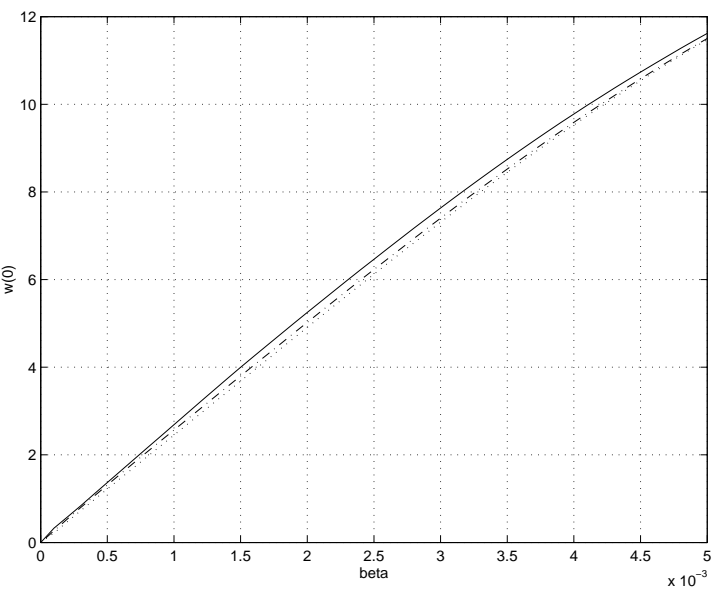

$G^{\prime}(u)=u^{7}$

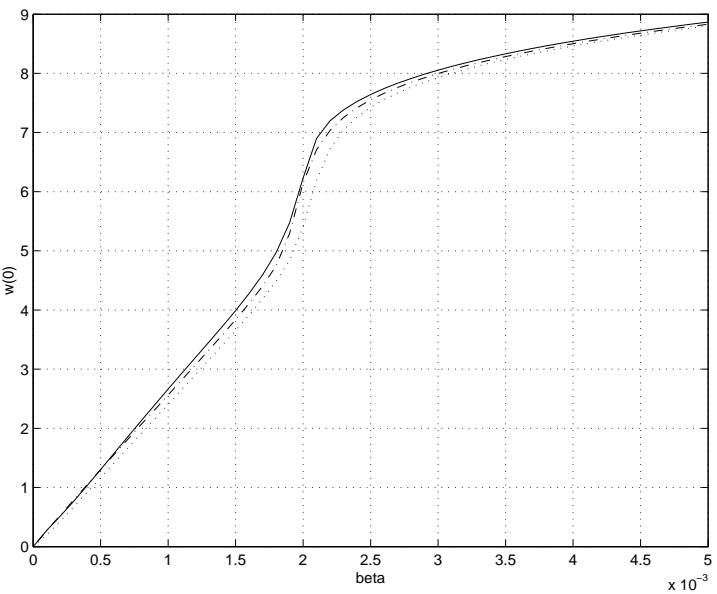

$G^{\prime}(u)=u^{3}$

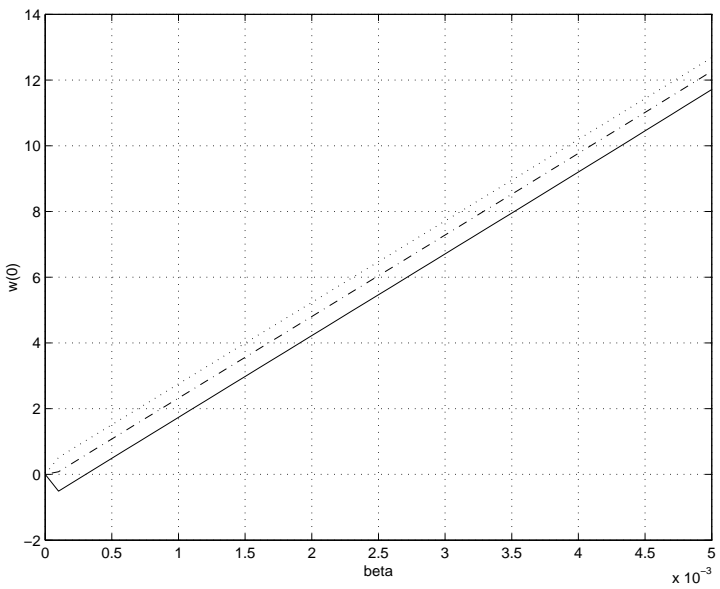

$G^{\prime}(u)=u^{5}$

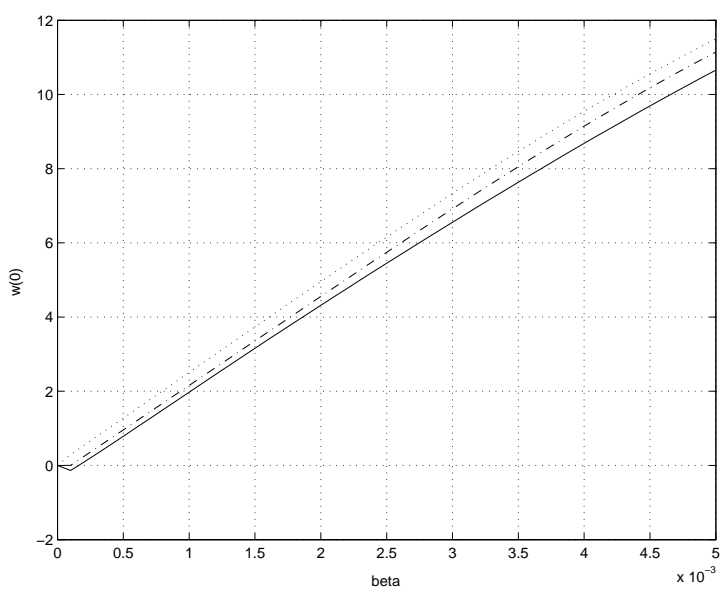

$G^{\prime}(u)=u^{7}$

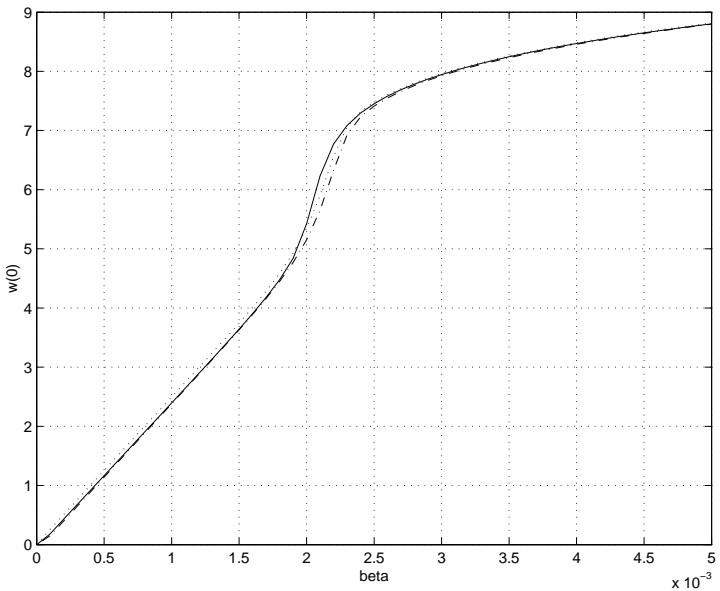

FIGURE 4.10: Approximate value of the solution to the Klein-Gordon equation near 0 vs. $\beta$ at $t=0.2$, for nonlinearity $G^{\prime}$ as indicated, $\gamma=0$ (solid), $\gamma=5$ (dashed) and $\gamma=10$ (dotted), initial data $\phi(r)=h(r), \psi(r)=h^{\prime}(r)+h(r) / r$ (left column), and $\phi(r)=0$ and $\psi(r)=100 h(r)$ (right column). 

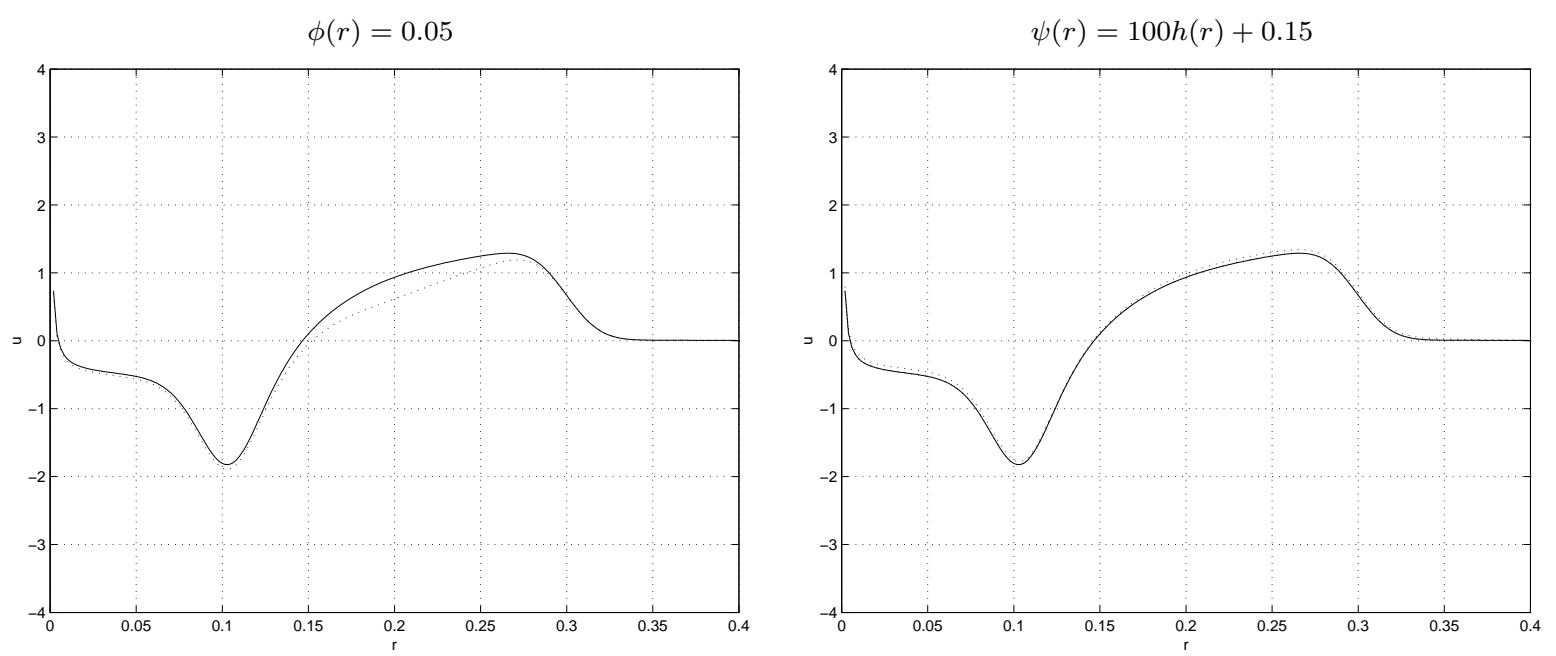

Figure 4.11: Approximate radial solutions for the Klein-Gordon equation with nonlinearity $G^{\prime}(u)=u^{5}$ at time $t=0.2$, for initial data $\phi(r)=0$ and $\psi(r)=100 h(r)$ (solid line), boundary condition $u(0.4, t)=0$, and the perturbation prescribed (dotted line).

As we observed before, the graphs of solutions corresponding to nonzero values of $\beta$ tend to increase near the origin for large values of $t$. To examine this more accurately, we have obtained the values of solutions of the modified nonlinear Klein-Gordon equation at $r=0.002, t=0.2$ and different values of $\beta$ and $\gamma$, for two different sets of initial conditions and exponents $p=3,5,7$ of the nonlinear term. The results are shown in Figure 4.10. The first column of graphs depicts the values of the approximate solution near 0 vs. $\beta$ for the choices $\gamma=0$ (solid line), $\gamma=5$ (dashed line), and $\gamma=10$ (dotted), and the initial data $\phi(r)=h(r), \psi(r)=h^{\prime}(r)+h(r) / r$. The second column show the corresponding values of the solution near 0 vs. $\beta$, but now for the initial data $\phi(r)=0$ and $\psi(r)=100 h(r)$. This figure shows that the value of the numeric solution near the origin for large values of $t$ tends to increase with $\beta$. The effect of $\gamma$ seems to be really small compared to the effect of $\beta$.

We have ran our algorithm for a long time, and we have been able to check that the values of the solutions near the origin tend to increase for fixed positive values of $\beta$. However, it seems that those values do not blow up to infinity; indeed, they seem to converge to some positive number. This agrees with the fact that the total energy is bounded by $E(0)$, but we have not been able to prove this formally.

It is interesting to observe the obvious bifurcation-like shift in the graphs of Figure 4.10 corresponding to $p=7$. We have noticed this behavior for several choices of initial data, even when $p=9$. We have proved that this shift does not correspond to a numerical stability of our scheme. To prove that, we have computed numerical solutions for different values of $\Delta r$ and $\Delta t$, and we have obtained similar results. One possible reason for this phenomenon is that for small values of $\beta$ the solutions of the modified Klein-Gordon equation resemble the solutions of the associated hyperbolic equation with $\beta$ equal to zero. For large values of $\beta$, though, the hyperbolic features of our equation are dropped and a new type of behavior is manifested in the solutions. This change is more pronounced for higher exponents $p$. Right or wrong, our results are in agreement with the theory for the nonlinear Klein-Gordon equation, which 
does not guarantee the existence and uniqueness of solutions for $p \geq 5$.

We finally wish to establish the stability of our method via a concrete example. Let us consider the initial data $\phi(r)=0$ and $\psi(r)=100 h(r)$, where the function $h$ is given as before, and let $\Delta r=0.002$ and $\Delta t=0.001$. The approximation to the exact solution of the modified nonlinear Klein-Gordon equation with nonlinear term given by $G^{\prime}(u)=|u|^{4} u$ at time 0.2 is depicted in Figure 4.11 in the form of a solid line. The problem of approximating the solution of the same nonlinear KleinGordon equation with the perturbed initial condition $\phi(r)=0.05$, keeping all other conditions unchanged yields the dotted-line graph in the left column of the same figure. It is evident that both solutions, perturbed and not perturbed, are very close indeed. We have obtained several graphs that show that smaller perturbations on this initial condition yield smaller variations with respect to the unperturbed solution. The values of the energy for the perturbed and the unperturbed case are very close indeed.

It is worthwhile to observe that similar conclusions are drawn when we consider the perturbed case $\psi(r)=100 h(r)+0.15$, keeping all other conditions unchanged. We conclude that our method seems to be conditionally stable.

\subsection{Discussion}

Our numerical analysis of the modified nonlinear Klein-Gordon equation with internal and external damping has led us to the following conclusions:

1. The relative difference of the solution of the modified nonlinear KleinGordon equation corresponding to a value of $\gamma$ with respect to the corresponding externally undamped solution increases as $\gamma$ increases (continuity of solutions with respect to $\gamma$ ).

2. For a fixed value of $\gamma$, the relative difference of the solution of the modified nonlinear Klein-Gordon equation corresponding to $\beta$ with respect to the corresponding internally undamped solution increases as $\beta$ increases (continuity of solutions with respect to $\beta$ ).

3. In the internally undamped case and for fixed large values of $t$, the larger the value of $\gamma$ the smaller the value of the amplitude at time $t$. Similarly, for a fixed value of $\gamma$, the larger the value of time the smaller the amplitude of the solutions.

4. The rate of change of energy with respect to time is negative. The larger the values of $\beta$ and $\gamma$ the more drastic the rate of decay of the total energy.

5. The loss of total energy for nonzero $\beta$ seems to follow an exponential-decay pattern. For positive values of $\beta$ we observe that

$$
\lim _{t \rightarrow \infty} E(t)=0 \quad \text { and } \quad \lim _{t \rightarrow-\infty} E(t)=\infty .
$$

For negative values of $\beta$ we see the opposite situation. 
6. For large values of time, the values of the solutions close to 0 tend to increase as $\beta$ increases. The effect of changing the value of $\gamma$ in those situations is almost imperceptible. Solutions do not seem to blow up in the neighborhood of 0 at finite time. 


\section{Appendix}

The numerical results were obtained using programs we especially wrote for Matlab 6.1. We give here the computational routines employed without the subroutines of graphical display.

The following routines were used to approximate radially symmetric solutions to the nonlinear Klein-Gordon equation with external damping and no internal damping. The method is implicit and makes use of Newton's method in each iteration to approximate the roots of a nonlinear equation.

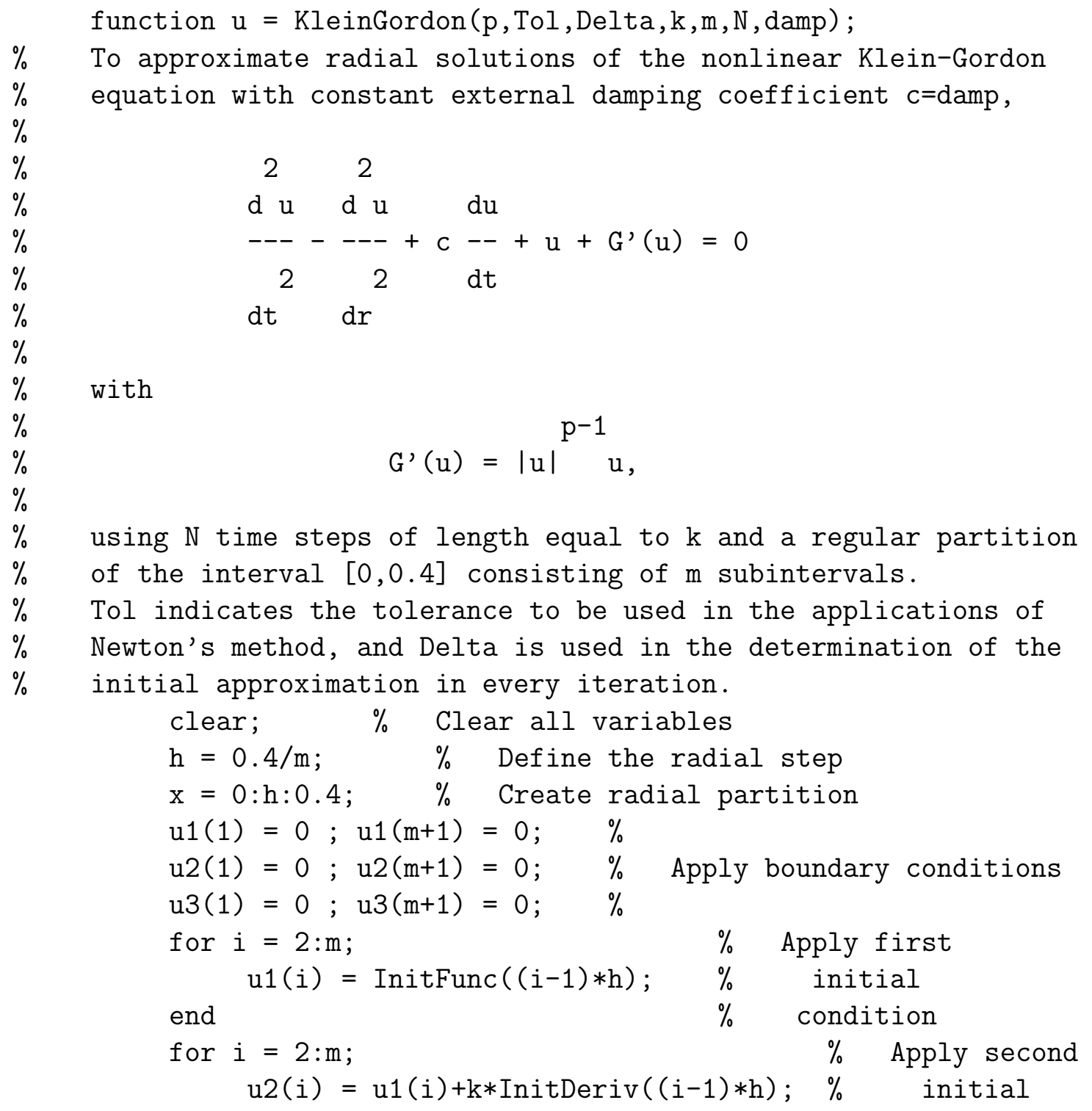


end

$\% \quad$ condition

for $\mathrm{j}=2: \mathrm{N} ; \quad \%$ Compute the next $\mathrm{N}-1$ approximations

for $i=2: \mathrm{m} ; \%$ Use Newton's method to approximate

$\%$ the solution in the $j$-th time step $\mathrm{u} 3(i)=$ Newton $(u 2(i-1), \mathrm{u} 2(i), \mathrm{u} 2(i+1), \mathrm{u} 1(i), \ldots$ $\mathrm{p}, \mathrm{h}, \mathrm{k}, \mathrm{i}-1, \mathrm{Tol}$, Delta , damp) ;

end

$\mathrm{E}=$ CalcEnergy $(\mathrm{u} 2, \mathrm{u} 3, \mathrm{~h}, \mathrm{k}, \mathrm{p}, \mathrm{m}) ; \%$ Calculate Energy $\mathrm{u} 1(:)=\mathrm{u} 2(:) ; \quad \%$ Update information vectors $\mathrm{u} 2(:)=\mathrm{u} 3(:) ; \quad \%$ for the next time step

end

$\mathrm{u}=\mathrm{u} 2 . / \mathrm{x}$;

function $\mathrm{x}=\operatorname{Newton}(\mathrm{a}, \mathrm{b}, \mathrm{c}, \mathrm{d}, \mathrm{p}, \mathrm{h}, \mathrm{k}, \mathrm{j}, \mathrm{Tol}$, Delta, damp);

$\% \quad$ Newton's method to approximate the solutions to the nonlinear

$\%$ equation

$\% \quad x-2 b+d \quad x-d \quad c-2 b+a \quad x+d \quad G(x)-G(d)$

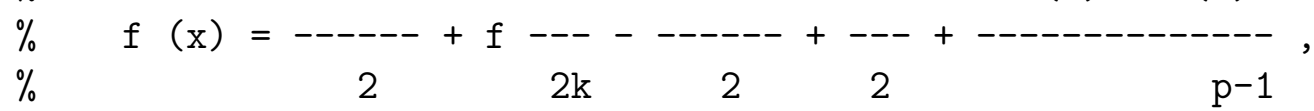

$\%$ k $\quad h \quad(j h(x-d))$

$\% \quad$ where $f=$ damp.

$\mathrm{Gd}=\operatorname{FuncG}(\mathrm{d}, \mathrm{p}, \mathrm{j}, \mathrm{h})$;

Diff $=1.0$;

$\mathrm{n}=1$;

$\% \quad$ Initialize difference

$\mathrm{x}=\mathrm{b}+$ Delta;

$\% \quad$ Initialize iterations

$\% \quad$ Initialize approximation

while (Diff > Tol) \& $(\mathrm{n}<25)$

$G x=\operatorname{FuncG}(x, p, j, h)$;

$\operatorname{DGx}=\operatorname{FuncDG}(\mathrm{x}, \mathrm{p}, \mathrm{j}, \mathrm{h})$;

$F x=F u n c F(x, a, b, c, d, p, h, k, j, G x, G d$, damp $) ; \%$ Newton's

$\mathrm{DFx}=\operatorname{FuncDF}(\mathrm{x}, \mathrm{d}, \mathrm{p}, \mathrm{h}, \mathrm{k}, \mathrm{j}, \mathrm{DGx}, \mathrm{Gx}, \mathrm{Gd}$, damp $) ; \%$ method

$\mathrm{x} 1=\mathrm{x}-\mathrm{Fx} / \mathrm{DFx} ; \quad \%$ Compute new approximation

$\mathrm{n}=\mathrm{n}+1 ; \quad \%$ Update counter

Diff $=\operatorname{abs}(x 1-x) ; \quad \%$ Compute difference

$\mathrm{x}=\mathrm{x} 1$;

end

function $\mathrm{y}=\operatorname{InitFunc}(r)$;

if $r<0.2$

$\mathrm{y}=5 * r * \exp \left(100 *\left(1-1 /\left(1-(10 * r-1)^{\wedge} 2\right)\right)\right) ;$

else

$\mathrm{y}=0$;

end

function $y=\operatorname{InitDeriv}(r)$;

if $r<0.195$

$\mathrm{y}=5 * r * \exp \left(100-5 /\left(\mathrm{r}-5 * r^{\wedge} 2\right)\right) *(5-49 * r-\ldots$ 


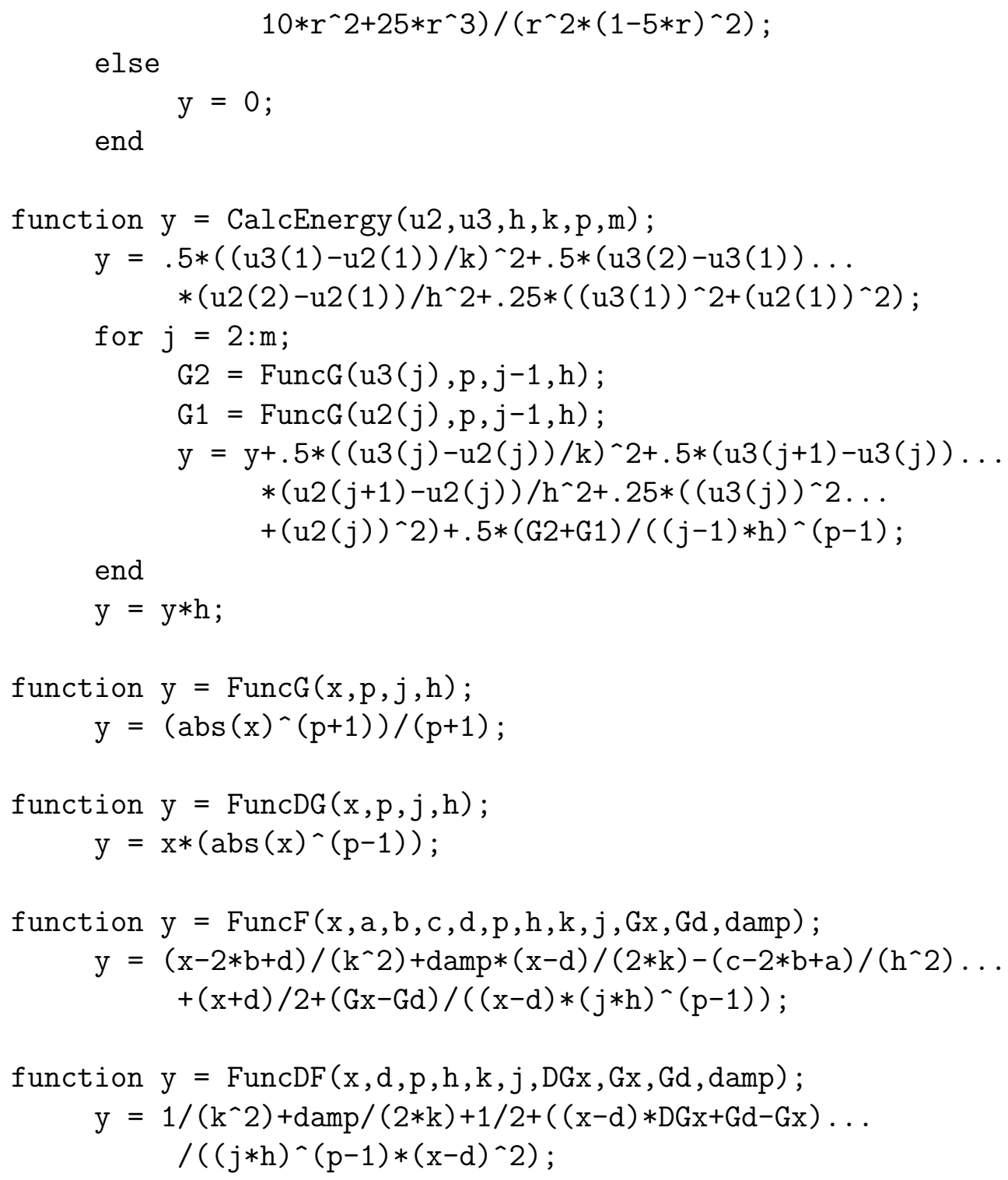

The following procedures were used to approximate radially symmetric solutions to the dissipative nonlinear Klein-Gordon equation. Our method is implicit and makes use of Newton's method with tolerance Tol to approximate the roots of a nonlinear system of equations in each iteration. In turn, each iteration of Newton's method makes use of Crout's reduction method for tridiagonal linear systems. The procedure CalcEnergy is the same one used in KleinGordon above. Likewise, all the routines used by KleinGordonMult which are not explicitly written below are assumed to be the same as those of KleinGordon.

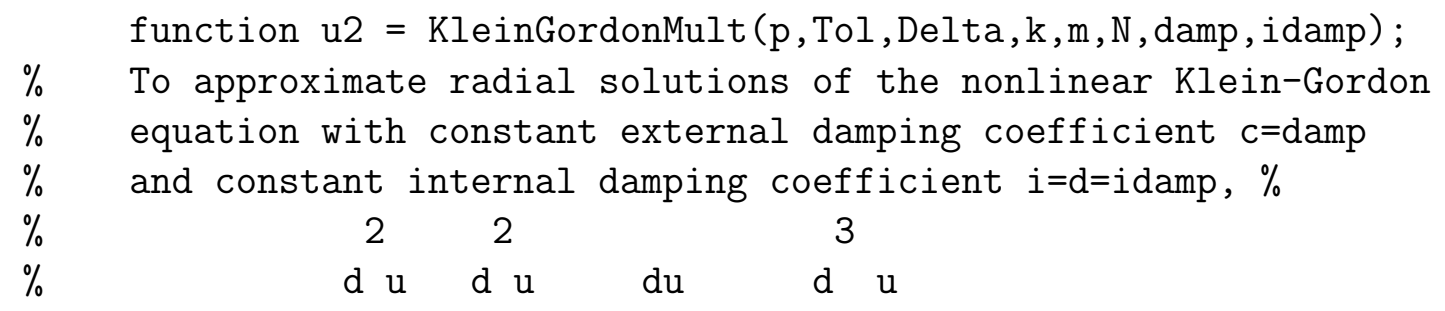


$\%$

$\%$

$\%$

$\%$

$\%$ with

$\%$

$\%$

$\%$

$\%$ using $\mathrm{N}$ time steps of length equal to $\mathrm{k}$ and a regular partition

$\%$ of the interval $[0,0.4]$ consisting of $\mathrm{m}$ subintervals.

$\%$ Tol indicates the tolerance to be used in the applications of

$\% \quad$ Newton's method, and Delta is used in the determination of the

$\% \quad$ initial approximation in every iteration.

$$
\begin{aligned}
& \text { clear; } \quad \% \text { Clear all variables } \\
& \mathrm{h}=0.4 / \mathrm{m} ; \quad \% \quad \text { Define the radial step } \\
& \mathrm{x}=0: \mathrm{h}: 0.4 ; \quad \% \quad \text { Create radial partition } \\
& \mathrm{u} 1(1)=0 ; \mathrm{u} 1(\mathrm{~m}+1)=0 ; \quad \% \\
& \mathrm{u} 2(1)=0 ; \mathrm{u} 2(\mathrm{~m}+1)=0 ; \quad \% \quad \text { Apply boundary conditions } \\
& \mathrm{u} 3(1)=0 ; \mathrm{u} 3(\mathrm{~m}+1)=0 ; \quad \% \\
& \text { for } i=2: m \text {; } \\
& \text { end } \\
& \mathrm{u} 1(\mathrm{i})=\operatorname{InitFunc}((\mathrm{i}-1) * \mathrm{~h}) ; \quad \% \quad \text { initial } \\
& \text { for } i=2: m \text {; } \\
& \% \quad \text { condition } \\
& \mathrm{u} 2(\mathrm{i})=\mathrm{u} 1(\mathrm{i})+\mathrm{k} * \operatorname{InitDeriv}((\mathrm{i}-1) * \mathrm{~h}) ; \% \text { initial }
\end{aligned}
$$

$\%$ condition

for $\mathrm{j}=2: \mathrm{N} ; \quad \% \quad$ Compute the next $\mathrm{N}-1$ approximations for $i=2: m$; $\%$ Use Newton's method to approximate

$\%$ the solution in the $j$-th time step u3(i) = NewtonMult (u1, u2, p, h, k, Tol, Delta, c, d, m);

end

$\mathrm{E}=$ CalcEnergy $(\mathrm{u} 2, \mathrm{u} 3, \mathrm{~h}, \mathrm{k}, \mathrm{p}, \mathrm{m}) ; \%$ Calculate Energy $\mathrm{u} 1(:)=\mathrm{u} 2(:) ; \quad \%$ Update information vectors $\mathrm{u} 2(:)=\mathrm{u} 3(:) ; \quad \% \quad$ for the next time step

end

$\mathrm{u}=\mathrm{u} 2 . / \mathrm{x}$

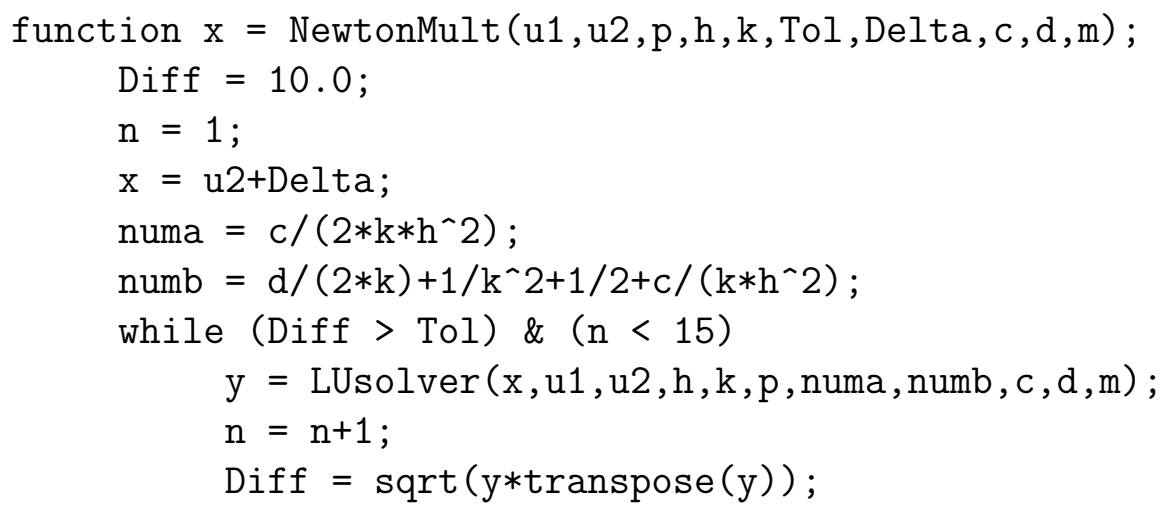




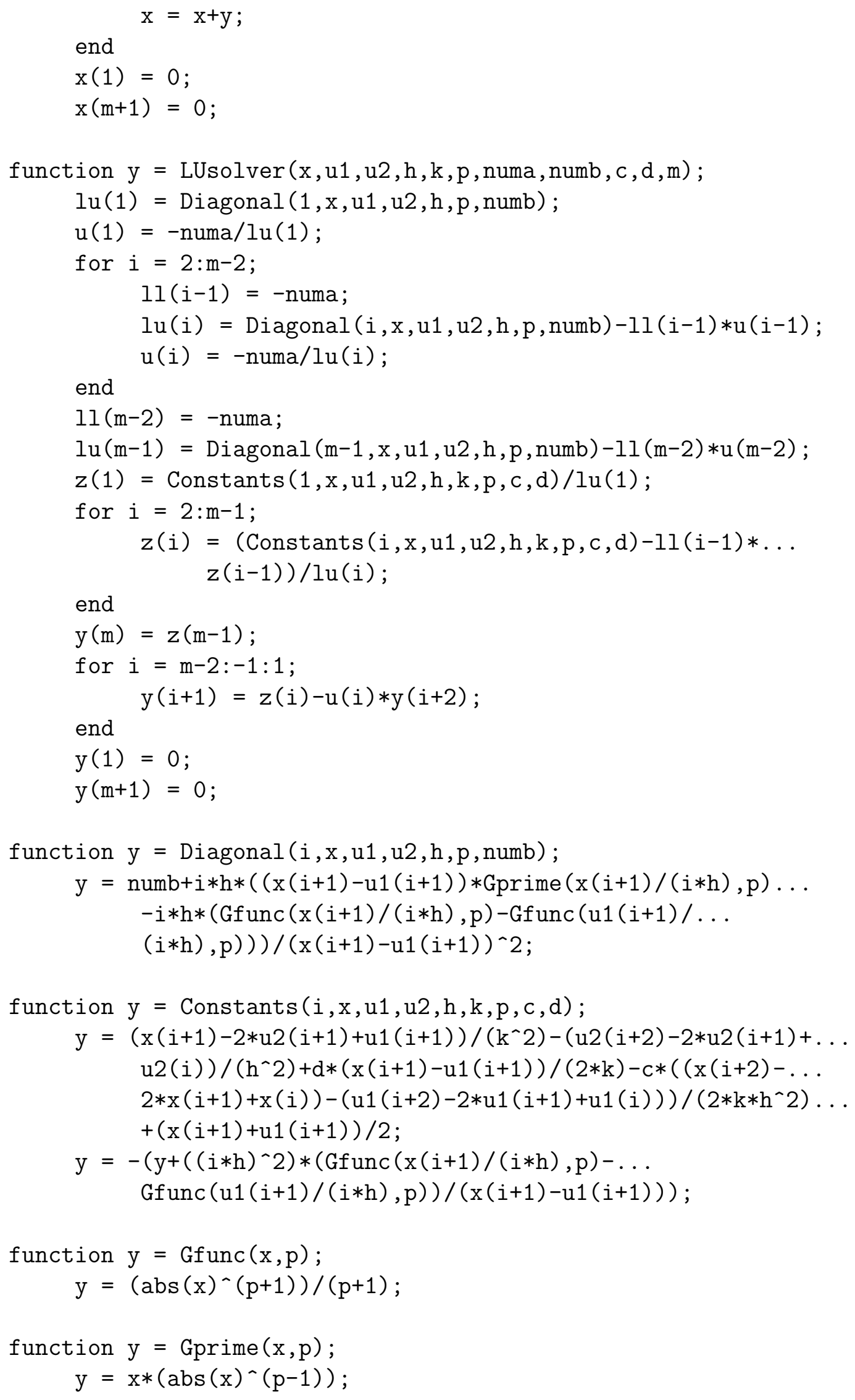




\section{References}

[1] Remoissenet, M., Waves Called Solitons, third ed., Springer-Verlag, New York, 1999.

[2] Lomdahl, P. S.; Soerensen, O. H.; Christiansen, P. L., "Soliton Excitations in Josephson Tunnel Junctions", Phys. Rev. B 25 (1982), no. 9, 5737-5748.

[3] Makhankov, V. G.; Bishop, A. R.; Holm, D. D. (ed.), Nonlinear Evolution Equations and Dynamical Systems Needs '94; Los Alamos, NM, USA 11-18 September '94: 10th International Workshop, first ed., World Scientific Pub. Co. Inc., Singapore, 1995.

[4] Polyanin, A. D.; Zaitsev, V. F., Handbook of Nonlinear Partial Differential Equations, first ed., Chapman \& Hall CRC Press, Boca Raton, Fla., 2004.

[5] Glassey, R. M., "Blow-up theorems for nonlinear wave equations", Math. Zeit. 132 (1973), 183-203.

[6] Jörgens, K, "Das Anfangswertproblem im Grossen fr eine Klasse nichtlinearer Wellengleichungen", Math. Zeit. 77 (1961), 295-308.

[7] Barone, A.; Esposito, F.; Magee, C. J.; Scott, A. C., "Theory and Applications of the Sine-Gordon Equation", Riv. Nuovo Cim. 1 (1971), 227-267.

[8] Strauss, W.; Vázquez, L., "Numerical Solution of a Nonlinear Klein-Gordon Equation", J. Comput. Phys. 28 (1978), 271-278.

[9] Alexander, F. J.; Habib, S., "Statistical Mechanics of Kinks in $1+1$ Dimensions", Phys. Rev. Lett. 71 (1993), 955-958.

[10] Zwillinger, D., Handbook of Differential Equations, third ed., Academic Press, Boston, MA, 1997.

[11] Perring, K. K.; Skyrme, T. H., "A Model Uniform Field Equation", Nucl. Phys. 31 (1962), 550-555.

[12] Tabor, M., Chaos and Integrability in Nonlinear Dynamics: An Introduction, John Wiley and Sons, New York, 1989. 
[13] Gibbon, J. D.; James, I. N.; Moroz, I. N., "The Sine-Gordon Equation as a Model for a Rapidly Rotating Baroclinic Fluid", Phys. Script. 20 (1979), 402-408.

[14] Bishop, A. R.; Schneider, T. (ed.), Solitons and Condensed Matter Physics, Springer-Verlag, New York, 1981.

[15] Fisher, R. A., "The Wave of Advance of Advantageous Genes", Annals of Eugenics 7 (1937), 355-369.

[16] Kolmogorov, A.; Petrovsky, I.; Piscounoff, N., "Étude de l'équations de la diffusion avec croissance de la quantité de matière et son application a un problème biologique", Bull. Univ. Moskou, Ser. Internat. 1A (1937), $1-25$.

[17] Sherratt, J. A.; Murray, J. D., "Models of Epidermal Wound Healing", Proc. Biol. Sci. 241 (1990), no. 1300, 29-36.

[18] Wallace, P. R., Mathematical Analysis of Physical Problems, first ed., Dover, New York, 1984.

[19] Rashba, E. I. and Sturge, M. D. (ed.), Excitons, North Holland, Amsterdam, 1982.

[20] Doering, C. R.; Mueller, C.; Smereka, P., "Interacting Particles, the FisherKolmogorov-Petrovsky-Piscounov Equation, and Duality", Physica A 325 (2003), 243-259.

[21] McGee, H.; McInerney, J.; and Harrus, A., "The Virtual Cook: Modeling Heat Transfer in the Kitchen", Physics Today 52 (1999), 30-36.

[22] Fourier, J. B. J. (ed.), Théorie Analytique de la Chaleur, Firmin Didot, Paris, 1822 .

[23] Cattaneo, M. C., "Sulla Conduzione de Calor", Atti Sem. Matem. e Fis. Della U. Modena 3 (1948), 3.

[24] Lieberstein, H. M., Theory of Differential Equations, first ed., Academic Press, New York, 1972.

[25] Segal, I. E., "The Global Cauchy problem for a relativistic scalar field with power interaction", Bull. Soc. Math. Fr. 91 (1963), 129-135.

[26] Morawetz, C. S.; Strauss, W. A., "Decay and scattering of solutions of a nonlinear relativistic wave equation", Comm. Pure and Appl. Math. 25 (1972), $1-31$. 
[27] Josephson, B. D., Ph.D. thesis, Cambridge University, Cambridge, England, 1964.

[28] Mason, W. P. (ed.), Physical Acoustics, Vol. III, first ed., Academic Press, New York, 1966.

[29] Krumhansl, J. A.; Schrieffer, J. R., "Dynamics and Statistical Mechanics of a One-Dimensional Model Hamiltonian for Structural Phase Transitions", Phys. Rev. B 11 (1975), 3535-3545.

[30] Currie, J. F.; Krumhansl, J. A.; Bishop, A. R.; Trullinger, S. E., "Statistical Mechanics of One-Dimensional Solitary-Wave-Bearing Scalar Fields: Exact Results and Ideal-Gas Phenomenology", Phys. Rev. B 22 (1980), 477-496.

[31] Royden, H., Real Analysis, third ed., Prentice Hall, New York, 1988.

[32] Thomas, J. W., Numerical Partial Differential Equations, first ed., SpringerVerlag, New York, 1995.

[33] Gelfand, I. M.; Fomin, S. V., Calculus of Variations, first ed., Dover Publications, Inc., Mineola, New York, 2000.

[34] Bradbury, T. C., Theoretical Mechanics, first ed., John Wiley \& Sons, Inc., New York, 1968.

[35] Burden, R. L.; Faires, J. D., Numerical Analysis, fourth ed., PWS-KENT Publishing Company, Boston, MA, 1989.

[36] Potter, M. M.; Morrey Jr., C. B., Intermediate Calculus, second ed., Undergraduate Texts in Mathematics, Springer-Verlag, New York, 1985.

[37] Strauss, W. A., Partial Differential Equations: An Introduction, second ed., John Wiley \& Sons, Inc., New York, 1992. 


\section{Index}

amplification matrix, 14

canonical Euler-Lagrange equation, 19

central second-order difference, 13

compact support, 3

consistency, 12

convergence, 12

Crout reduction method, 34

Crout's reduction method, 35

damped equation, 5

damping coefficient of external, 5 term of external, 5 term of internal, 8

diffusion equation, 4 in three dimensions, 4

discrete Fourier transform, 13

discriminant, 4

domain, 3

elliptic equation, 4

Fisher-KPP equation, 6 stochastic, 6

Fourier equation, 7

function

small at infinity, 3

smooth, 3

gradient

spherical coordinates, 19

Green's first identity, 20

heat equation, 4

Helmholtz equation, 5

hyperbolic equation, 4

Jacobian matrix, 35

Josephson

current, 9

effect, 10 interferometer, 9

juntion, 9

tunneling, 9

kinks, 10

Klein-Gordon equation

linear, 6

modified, 29

nonlinear

damped, 8

dissipative, 8

Langevin equation, 10

Laplace's equation, 4

in three dimensions, 5

Laplacian, 4

Lax theorem, 14

linear equation, 5

matrix norm, 12

Maxwell-Cattaneo equation, 7

Newton's method, 17

for nonlinear systems, 34

noise, 6

Gaussian, 6

white, 6

norm, 11

$p$-, 11

Euclidean, 11

infinity, 11

taxicab, 11

normed linear space, 11

parabolic equation, 4

phonon, 10

Poisson's equation, 5

Schrödinger's equation, 5

sine-Gordon equation, 6

space

$L_{p}(X), 12$

$\ell_{p}, 12$ 
$\ell_{p, \Delta x}, 12$

energy, 12

stability

conditional, 13

unconditional, 13

stability order $n, 13$

telegraph equation, 7

undamped equation, 5

wave equation, 4

in three dimensions, 4 


\section{Vita}

Jorge Eduardo Macías Díaz was born in the city of Aguascalientes, of the Mexican state of the same name, on June 19, 1970. He obtained his diploma of bachelor in science in applied mathematics from the Universidad Autónoma de Aguascalientes in the Spring semester of 1994. Mr. Macías Díaz obtained a master of science degree in mathematics from Tulane University in the Spring semester of 1998. He continued his studies in this same institution and was awarded a Ph.D. degree in mathematics in the Spring semester of 2001. Jorge Eduardo expects to obtain his master of science in applied physics from the University of New Orleans in Summer of 2004 . 